\title{
Conformal bootstrap to Rényi entropy in 2D Liouville and super-Liouville CFTs
}

\author{
Song $\mathrm{He}^{*}$ \\ Center for Theoretical Physics and College of Physics, Jilin University, \\ Changchun 130012, People's Republic of China \\ and Max Planck Institute for Gravitational Physics (Albert Einstein Institute), \\ Am Mühlenberg 1, 14476 Golm, Germany
}

(Received 30 June 2018; published 7 January 2019)

\begin{abstract}
The Rényi entanglement entropy (REE) of the states excited by local operators in two-dimensional irrational conformal field theories (CFTs), especially in Liouville field theory (LFT) and $\mathcal{N}=1$ superLiouville field theory (SLFT), has been investigated. In particular, the excited states obtained by acting on the vacuum with primary operators were considered. We start from evaluating the second REE in a compact $c=1$ free boson field theory at generic radius, which is an irrational CFT. Then we focus on the two special irrational CFTs, e.g., LFT and SLFT. In these theories, the second REE of such local excited states becomes divergent in early and late time limits. For simplicity, we study the memory effect of REE for the two classes of the local excited states in LFT and SLFT. In order to restore the quasiparticles picture, we define the difference of REE between target and reference states, which belong to the same class. The variation of the difference of REE between early and late time limits always coincides with the log of the ratio of the fusion matrix elements between target and reference states. Furthermore, the locally excited states by acting generic descendent operators on the vacuum have been also investigated. The variation of the difference of REE is the summation of the log of the ratio of the fusion matrix elements between the target and reference states and an additional normalization factor. Since the identity operator (or vacuum state) does not live in the Hilbert space of LFTand SLFT and no discrete terms contribute to REE in the intermediate channel, the variation of the difference of REE between target and reference states is no longer the log of the quantum dimension which is shown in the $1+1$-dimensional rational CFTs (RCFTs).
\end{abstract}

DOI: $10.1103 /$ PhysRevD.99.026005

\section{INTRODUCTION}

One can define some observables to detect the property of the vacuum or excited states in a local quantum field theory. For example, entanglement entropy (EE) and the Rényi entanglement entropy (REE) are helpful quantities to use when studying global or nonlocal structures in QFTs. For a subsystem, the entanglement entropy and Rényi entropy, both of them are defined as a function of the reduced density matrix $\rho_{A}$, which can be obtained by tracing out the degrees of freedom of the complement of $A$ in the original density matrix $\rho$.

One might wonder whether there is a kind of topological contribution to the entanglement entropy even for gapless theories, e.g., conformal field theories (CFTs) (e.g.,

\footnotetext{
*hesong17@gmail.com
}

Published by the American Physical Society under the terms of the Creative Commons Attribution 4.0 International license. Further distribution of this work must maintain attribution to the author(s) and the published article's title, journal citation, and DOI. Funded by SCOAP ${ }^{3}$. computing topological contributions in entanglement entropy called topological entanglement entropy [1] can quantify some topological properties). Authors of an earlier work [2] pointed out a connection between the topological entanglement entropy and boundary entropy. Furthermore, the connection between the boundary entropy and entanglement entropy was explored in [3]. Previously, the authors of [4] found that the entanglement entropy of local excited states has a connection with the quantum dimension in rational CFTs. In this paper, we would like to check whether the Rényi and von Neumann entropies of locally excited states are still topological quantities or not in twodimensional irrational CFTs.

The $n$th Rényi entanglement entropy $S_{A}^{(n)}$ is defined by $S_{A}^{(n)}=\log \operatorname{Tr}\left[\rho_{A}^{n}\right] /(1-n)$. By analytical continuation of $n$, the $S_{A}^{(n)}$ coincides with the von Neumann entropy in the limit $n \rightarrow 1$. By using the so-called standard replica trick, one can calculate the entanglement entropy in field theory. One can extend [3] from vacuum states to locally excited states in CFTs. The computations of entanglement entropies for local excited states have been carried out in [5-7] 
in various dimensional field theories. The entanglement entropy for free scalar fields has been investigated in [7-9]. In large $n$ CFTs with holographic dual, the entanglement entropy for locally excited states has been studied in $[10,11]$. This study mainly focuses on the variation of Rényi entropy $S_{A}^{(n)}$ between excited states and a reference state, where the excited states are acquired by acting primary or descendent fields on the vacuum in irrational CFTs. The variation of n-th REE is denoted by $\Delta S_{A}^{(n)}$.

In 2D rational CFTs, it was found [4] that, for the locally primary excited states, the variation of $n$th Rényi entanglement entropy is related to the quantum dimension $[12,13]$ of the associated primary operator. The quantum dimension is the measure of the effective degrees of freedom of a local operator and it is a kind of topological quantity. In various dimensional CFTs, REE has been studied in [14-34] from various perspectives. The papers $[16,17,19,20]$ mainly concentrated on entanglement entropy in higherdimensional field theory. The authors of [18] have found the REE of local excited states in large central charge $1+1$-dimensional CFTs from holography. In particular, the authors of $[14,20]$ have provided a perspective from which to study Rényi entanglement entropy from string theory and it provides us with a one loop correction to the large black hole entropy. In [21,22], the entanglement entropy of a local excited state in some specific quantum Lifshitz models has been presented. More recently, the authors of [35] mainly studied the local states of the product form of local operators in rational CFTs and they found that the variation of REE is consistent with the scattering process during entanglement propagation in RCFTs.

In this research, the previous study $[4,15,36]$ on the Rényi entanglement entropy for the primary and descendent states has been generalized to irrational CFTs, especially for Liouville field theory (LFT) and super-Liouville field theory (SLFT). Previously, the authors of [37] studied the memory effect of REE in a compact $c=1$ free boson theory at a generic radius, which is an irrational CFT. There are two main motivations driving the research in LFT and SLFT. The first one is that the representation of the spectra will be infinite dimensional in irrational CFTs; therefore, extracting entanglement entropy for local excited states will be highly nontrivial. A priori, one cannot expect that the variation of REE will still be the log of the quantum dimension. Furthermore, the quantum dimension of a local primary operator in irrational CFTs will be quite different from that in the 1+1-dimensional rational CFTs. Discovering how to measure the variation of REE in irrational CFTs in a precise, robust way is our main aim. The second is that LFT can be reformulated as 3D Chern Simons theory [38] or 3D gravity theory. In the large central charge limit, the Liouville field theory might have AdS/ CFT-like connections [39-41] with 3D gravity. Basically, the boundary conditions in Chern-Simons theory are associated with the Virasoro conformal blocks. The Liouville primary fields can be regarded as monodromy defects, which was proposed in [42]. To understand whether or not these connections are AdS/CFT-like, we would like to work out the large central charge properties of local excited states by primary fields in LFT or SLFT; because EE and REE can be probed on the field theory side and the holographic side, both of them will be good objects with which to test the properties of these connections. In this sense, the large $c$ universal properties from these data can be generated to compare with the holographic expectation [10] of REE.

In this paper, we evaluate the second REE in a compact $c=1$ free boson field theory at generic radius, which is an irrational CFT, as a preliminary exercise to test the memory effect of REE. Then we mainly study the $1+1$ dimensional LFT and SLFT to show how to extract the variation of REE for locally excited states between the early time limit and the late time limit. The second REE of local primary excited states by using CFT techniques is shown in a precise way; then these calculations can be extended to the $n$th REE of primary and descendent states following $[4,36]$. From these studies, we find that the REE of local excited states in LFT and SLFT is divergent, which is consistent with the classifications of local operators in LFT [43]. By choosing an appropriate reference state $V_{\alpha_{r}}|0\rangle$, we redefine a new quantity $\Delta S_{\mathrm{EE}}^{(n)}\left[V_{\alpha}|0\rangle, V_{\alpha_{r}}|0\rangle\right]$ as the difference of REE between target and reference states to measure the time evolution of REE in LFT and SLFT, which is consistent with the quasiparticle picture given in rational CFTs. This difference of REE $\Delta S_{\mathrm{EE}}^{(n)}\left[V_{\alpha}|0\rangle, V_{\alpha_{r}}|0\rangle\right]$ can be reduced to the $\Delta S_{\mathrm{EE}}^{(n)}$ [4] given in rational CFTs by choosing the vacuum state as a reference state. Finally, the variation of the difference of REE $\Delta S_{\mathrm{EE}}^{(n)}\left[V_{\alpha}|0\rangle, V_{\alpha_{r}}|0\rangle\right](t \rightarrow \infty)-$ $\Delta S_{\mathrm{EE}}^{(n)}\left[V_{\alpha}|0\rangle, V_{\alpha_{r}}|0\rangle\right](t \rightarrow 0)$ depends on the ratio of the fusion matrix elements associated with $V_{\alpha}, V_{\alpha_{r}}$ in LFT and SLFT, unlike that of rational CFTs, which cannot be identified with the quantum dimension. That is to say, $\Delta S_{\mathrm{EE}}^{(n)}\left[V_{\alpha}|0\rangle, V_{\alpha_{r}}|0\rangle\right](t \rightarrow \infty)-\Delta S_{\mathrm{EE}}^{(n)}\left[V_{\alpha}|0\rangle, V_{\alpha_{r}}|0\rangle\right](t \rightarrow 0)$ will depend on the details of LFT and SLFT.

The outline of this paper is as follows. In Sec. II, we give the $1+1$-dimensional setup and study the second REE in a precise way in LFT and $\mathcal{N}=1$ SLFT. The difference of REE between the target state and reference state $\Delta S_{\mathrm{EE}}^{(2)}\left[V_{\alpha}|0\rangle, V_{\alpha_{r}}|0\rangle\right]$ has been calculated. In Sec. III, we extend the above calculation of second Rényi entropy to the $n$th REE in LFT to show that the variations of REE are the log of the fusion matrix elements ratio, which are quite different from those in the rational CFTs. In Sec. IV, the difference of REE $\Delta S_{\mathrm{EE}}^{(n)}\left[V_{\alpha}|0\rangle, V_{\alpha_{r}}|0\rangle\right]$ between states generated by acting descendent operators on the vacuum state in this setup is studied. Finally, we devote Sec. V to the conclusions and discussions and also mention some likely future problems. In the Appendices, we list some relevant notations and techniques which are necessary to our analysis. 


\section{THE SECOND RÉNYI ENTANGLEMENT ENTROPY}

\section{A. Setup in 2D CFT}

An excited state is defined by an operator $\mathcal{O}_{a}$ acting on the vacuum $|0\rangle$ in a two-dimensional CFT. The operator can be primary or descendent. We can make use of the Euclidean formulation and introduce the complex coordinate $(w, \bar{w})=(x+i \tau, x-i \tau)$ on $R^{2}$ such that $\tau$ and $x$ denote Euclidean time and space, respectively. We introduce operator $O_{a}$ at $x=-l<0$ initially and investigate its real time evolution from time 0 to $t$ under the Hamiltonian $H$. We develop the setup shown in Fig. 1 and the corresponding density matrix reads as follows:

$$
\begin{aligned}
\rho(t) & =C_{a} \cdot e^{-i H t} e^{-\epsilon H} O_{a}(-l)|0\rangle\langle 0| O_{a}^{\dagger}(-l) e^{-\epsilon H} e^{i H t} \\
& =C_{a} \cdot O_{a}\left(w_{2}, \bar{w}_{2}\right)|0\rangle\langle 0| O_{a}^{\dagger}\left(w_{1}, \bar{w}_{1}\right),
\end{aligned}
$$

where $C_{a}$ is determined by requiring $\operatorname{Tr} \rho(t)=1$. Here we can define coordinates as

$$
\begin{aligned}
& w_{1}=i(\epsilon-i t)-l, \quad w_{2}=-i(\epsilon+i t)-l, \\
& \bar{w}_{1}=-i(\epsilon-i t)-l, \quad \bar{w}_{2}=i(\epsilon+i t)-l .
\end{aligned}
$$

$\epsilon$ is an infinitesimal positive parameter as an ultraviolet regulator. Until the end of the calculations, we treat $\epsilon \pm i t$ as purely imaginary numbers as in $[4,7,8]$.

To calculate variation of the $n$th REE $\Delta S_{A}^{(n)}$, we employ the replica method in the path-integral formalism ${ }^{1}$ by generalizing the formulation for the ground states [3] to excited states [7]. In this paper, we choose the subsystem $A$ to be an interval $0 \leq x \leq L$ at $\tau=0$. For simplification, we only consider $L \rightarrow \infty$ throughout. It leads to an $n$-sheeted Riemann surface $\Sigma_{n}$ with $2 n$ operators $\mathcal{O}_{a}$ inserted.

Finally, the $\Delta S_{A}^{(n)}$ can be calculated as

$$
\begin{aligned}
\Delta S_{A}^{(n)}= & \frac{1}{1-n}\left[\log \left\langle O_{a}^{\dagger}\left(w_{1}, \bar{w}_{1}\right) O_{a}\left(w_{2}, \bar{w}_{2}\right) \cdots O_{a}\left(w_{2 n}, \bar{w}_{2 n}\right)\right\rangle_{\Sigma_{n}}\right. \\
& \left.-n \log \left\langle O_{a}^{\dagger}\left(w_{1}, \bar{w}_{1}\right) O_{a}\left(w_{2}, \bar{w}_{2}\right)\right\rangle_{\Sigma_{1}}\right],
\end{aligned}
$$

where $\left(w_{2 k+1}, w_{2 k+2}\right)$ for $k=1,2, \ldots, n-1$ are $n-1$ replicas of $\left(w_{1}, w_{2}\right)$ in the $k$ th sheet of $\Sigma_{n}$. The term in the first line in Eq. (4) is given by a $2 n$-point correlation function on $\Sigma_{n}$. Here $\Delta_{a}$ is the (chiral and antichiral) conformal dimension of the operator $O_{a}$. One should note that $\Delta S_{A}^{(n)}$ can be well defined once the vacuum state belongs to the Hilbert space. That is to say, one can choose the vacuum state as a good reference state to measure $\Delta S_{A}^{(n)}=\Delta S_{A}^{(n)}\left[V_{\alpha}|0\rangle,|0\rangle\right]$ between excited states and the vacuum state in rational CFTs [4]. Otherwise, one has to choose an appropriate reference state $V_{\alpha_{r}}|0\rangle$ to measure $\Delta S_{A}^{(n)}\left[V_{\alpha}|0\rangle, V_{\alpha_{r}}|0\rangle\right]$, e.g., in Liouville field theory. ${ }^{2}$

\footnotetext{
${ }^{1}$ More precisely, the replica method for the local operator excited states in field theory has been explicitly shown in Sec. 2.2 of [35].

${ }^{2}$ We will explain the details at the end of this section.
}

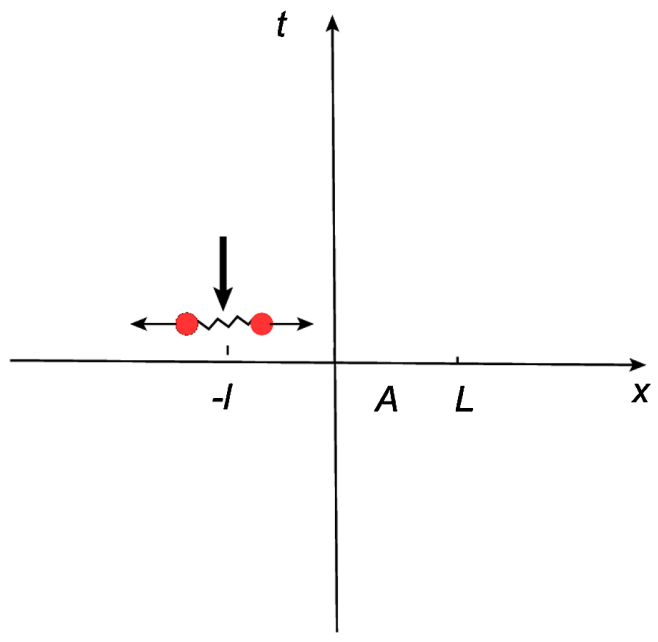

FIG. 1. This figure is to show our basic setup in the twodimensional plane $w=x+i t$. We consider the subsystem $A$, $0<x<L$, with $L \rightarrow \infty$. We just put the local operators at $x=-l, t=0$. The local operators will trigger left- and rightmoving quasiparticles with time evolution.

\section{B. Convention}

Firstly, we study $n=2$, i.e., the second Rényi entanglement entropy, in detail. The calculation of $\Delta S_{A}^{(2)}$ is reduced to four-point functions in CFTs.

For $n=2$, one can connect the coordinate $w_{i}$ with $z_{i}$ by a conformal mapping $w_{i}=z_{i}^{2}$, which looks like

$$
\begin{aligned}
& w_{1}=i \epsilon+t-l \equiv r e^{i \theta_{1}}=\left(z_{1}\right)^{2}, \\
& w_{2}=-i \epsilon+t-l \equiv s e^{i \theta_{2}}=\left(z_{2}\right)^{2}, \\
& w_{3}=(i \epsilon+t-l) e^{2 \pi i} \equiv r e^{i\left(2 \pi+\theta_{1}\right)}=\left(z_{3}\right)^{2}, \\
& w_{4}=(-i \epsilon+t-l) e^{2 \pi i} \equiv s e^{i\left(2 \pi+\theta_{2}\right)}=\left(z_{4}\right)^{2} .
\end{aligned}
$$

Thus one can find

$$
\begin{aligned}
& z_{1}=-z_{3}=\sqrt{w_{1}}=\sqrt{r} e^{i \theta_{1} / 2}=i \sqrt{l-t-i \epsilon} \\
& z_{2}=-z_{4}=\sqrt{w_{2}}=\sqrt{s} e^{i \theta_{2} / 2}=i \sqrt{l-t+i \epsilon} .
\end{aligned}
$$

If readers are interested in the finite size formula, please refer to [4].

We will follow the standard procedure of the analytical continuation of Euclidean theory into its Lorentzian version. The most important and subtle point is that we should treat $\pm i \epsilon+t$ as a pure imaginary number in all algebraic calculations. Finally, we take $t$ to be real only in the final expression of the variation of entropy. Here we identify

$$
\begin{aligned}
& \left(r \cos \theta_{1}, r \sin \theta_{1}\right)=(-l, \epsilon-i t), \\
& \left(s \cos \theta_{2}, s \sin \theta_{2}\right)=(-l,-\epsilon-i t),
\end{aligned}
$$

which leads to 


$$
\begin{aligned}
r & =\sqrt{l^{2}+(\epsilon-i t)^{2}}, \quad s=\sqrt{l^{2}+(-\epsilon-i t)^{2}}, \\
r s & =\sqrt{\left(l^{2}+\epsilon^{2}-t^{2}\right)^{2}+4 \epsilon^{2} t^{2}}, \\
r^{2}+s^{2} & =2\left(l^{2}+\epsilon^{2}-t^{2}\right), \\
\cos \left(\theta_{1}-\theta_{2}\right) & =2 \cos ^{2}\left(\frac{\theta_{1}-\theta_{2}}{2}\right)-1 \\
& =\frac{l^{2}-\epsilon^{2}-t^{2}}{\sqrt{\left(l^{2}+\epsilon^{2}-t^{2}\right)^{2}+4 \epsilon^{2} t^{2}}} .
\end{aligned}
$$

To get REE, we solely focus on the conformal cross ratio

$$
\begin{aligned}
& z=\frac{z_{12} z_{34}}{z_{13} z_{24}}=\frac{-(l-t)+\sqrt{(l-t)^{2}+\epsilon^{2}}}{2 \sqrt{(l-t)^{2}+\epsilon^{2}}}, \\
& \bar{z}=\frac{\bar{z}_{12} \bar{z}_{34}}{\bar{z}_{13} \bar{z}_{24}}=\frac{-(l+t)+\sqrt{(l+t)^{2}+\epsilon^{2}}}{2 \sqrt{(l+t)^{2}+\epsilon^{2}}},
\end{aligned}
$$

where $z_{i j}=z_{i}-z_{j}$.
It is useful to note the relationship

$$
1-z=\frac{z_{14} z_{23}}{z_{13} z_{24}}
$$

We are interested in the two limits (i) $l \gg t \gg \epsilon$ (early time) and (ii) $t \gg l \gg \epsilon$ (late time), and from (9) we can know that they separately correspond to

$$
\begin{aligned}
& \text { (i) } z \simeq \bar{z} \simeq \frac{\epsilon^{2}}{4 l^{2}}(\rightarrow 0), \\
& \text { (ii) } z \simeq 1-\frac{\epsilon^{2}}{4 t^{2}}(\rightarrow 1), \quad \bar{z} \simeq \frac{\epsilon^{2}}{4 t^{2}}(\rightarrow 0) .
\end{aligned}
$$

Note that the late time limit is quite nontrivial, which originates from our analytical continuation of $t$.

\section{The variation of second Rényi entanglement entropy}

The four-point function on $\Sigma_{2}$ is mapped onto that of $R^{2}$ by the conformal map $w=z^{2}$. Thus we find

$$
\begin{aligned}
\left\langle O_{a}\left(w_{1}, \bar{w}_{1}\right) O_{a}\left(w_{2}, \bar{w}_{2}\right) O_{a}\left(w_{3}, \bar{w}_{3}\right) O_{a}\left(w_{4}, \bar{w}_{4}\right)\right\rangle_{\Sigma_{2}} & =\prod_{i=1}^{4}\left|\frac{d w_{i}}{d z_{i}}\right|^{-2 \Delta}\left\langle O_{a}\left(z_{1}, \bar{z}_{1}\right) O_{a}\left(z_{2}, \bar{z}_{2}\right) O_{a}\left(z_{3}, \bar{z}_{3}\right) O_{a}\left(z_{4}, \bar{z}_{4}\right)\right\rangle_{\Sigma_{1}} \\
& =2^{-8 \Delta}\left|z_{1} z_{2} z_{3} z_{4}\right|^{-2 \Delta} \cdot\left\langle O_{a}\left(z_{1}, \bar{z}_{1}\right) O_{a}\left(z_{2}, \bar{z}_{2}\right) O_{a}\left(z_{3}, \bar{z}_{3}\right) O_{a}\left(z_{4}, \bar{z}_{4}\right)\right\rangle_{\Sigma_{1}} \\
& =2^{-8 \Delta} \cdot(r s)^{-2 \Delta} \cdot\left\langle O_{a}\left(z_{1}, \bar{z}_{1}\right) O_{a}\left(z_{2}, \bar{z}_{2}\right) O_{a}\left(z_{3}, \bar{z}_{3}\right) O_{a}\left(z_{4}, \bar{z}_{4}\right)\right\rangle_{\Sigma_{1}},
\end{aligned}
$$

where $\Delta$ is the chiral conformal dimension of the operator $O_{a}$.

The two-point function looks like

$$
\left\langle O_{a}\left(w_{1}, \bar{w}_{1}\right) O_{a}\left(w_{2}, \bar{w}_{2}\right)\right\rangle_{\Sigma_{1}}=\frac{C_{a}}{\left|w_{12}\right|^{4 \Delta}}=\frac{C_{a}}{(2 \epsilon)^{4 \Delta}},
$$

where $C_{a}$ represents normalization. Note that the four-point function is proportional to $C_{a}^{2}$ and the $\Delta S_{A}^{(2)}$ is of course independent of $C_{a}$. Thanks to the conformal symmetry, the four-point function on $R^{2}$ can be expressed as

$$
\left\langle O_{a}\left(z_{1}, \bar{z}_{1}\right) O_{a}\left(z_{2}, \bar{z}_{2}\right) O_{a}\left(z_{3}, \bar{z}_{3}\right) O_{a}\left(z_{4}, \bar{z}_{4}\right)\right\rangle_{\Sigma_{1}}=\left|z_{13} z_{24}\right|^{-4 \Delta} \cdot G(z, \bar{z}),
$$

where $(z, \bar{z})$ are given by (9).

In the late time limit (ii), we finally find that the ratio in (16) is expressed in terms of the four-point function on $R^{2}$ :

$$
\begin{aligned}
\operatorname{Tr} \rho_{A}^{2}=\frac{\left\langle O_{a}\left(w_{1}, \bar{w}_{1}\right) O_{a}\left(w_{2}, \bar{w}_{2}\right) O_{a}\left(w_{3}, \bar{w}_{3}\right) O_{a}\left(w_{4}, \bar{w}_{4}\right)\right\rangle_{\Sigma_{2}}}{\left(\left\langle O_{a}\left(w_{1}, \bar{w}_{1}\right) O_{a}\left(w_{2}, \bar{w}_{2}\right)\right\rangle_{\Sigma_{1}}\right)^{2}} & \simeq \frac{1}{C_{a}^{2}} \cdot\left(\frac{\epsilon^{2}}{t}\right)^{4 \Delta} \cdot\left\langle O_{a}\left(z_{1}, \bar{z}_{1}\right) O_{a}\left(z_{2}, \bar{z}_{2}\right) O_{a}\left(z_{3}, \bar{z}_{3}\right) O_{a}\left(z_{4}, \bar{z}_{4}\right)\right\rangle_{\Sigma_{1}} \\
& \simeq \frac{1}{C_{a}^{2}}\left|z^{4 \Delta}(1-z)^{4 \Delta}\right| G(z, \bar{z}) \simeq \frac{1}{C_{a}^{2}} \cdot\left(\frac{\epsilon^{2}}{4 t^{2}}\right)^{4 \Delta} \cdot G(z, \bar{z}) .
\end{aligned}
$$

In rational CFTs, we can calculate $\Delta S_{A}^{(n)}$ between local excited states and the vacuum state as follows:

$$
\Delta S_{A}^{(n)}=S_{A}^{(n)}\left(O_{a}|0\rangle\right)-S_{A}^{(n)}(1|0\rangle)=\frac{1}{1-n} \log \left[\frac{\left\langle O_{a}\left(w_{1}, \bar{w}_{1}\right) O_{a}\left(w_{2}, \bar{w}_{2}\right) \cdots O_{a}\left(w_{2 n}, \bar{w}_{2 n}\right)\right\rangle_{\Sigma_{n}}}{\left(\left\langle O_{a}\left(w_{1}, \bar{w}_{1}\right) O_{a}\left(w_{2}, \bar{w}_{2}\right)\right\rangle_{\Sigma_{1}}\right)^{n}}\right] .
$$

Here $\Sigma_{n}$ denotes the $n$-sheeted Euclidean surface given by the metric

$$
d s^{2}=d \rho^{2}+\rho^{2}(d \theta)^{2},
$$

where $\theta$ has the $2 \pi n$ periodicity $\theta \sim \theta+2 \pi n$. 
Extra care should be taken when we generalize (16) to the case of LFT and SLFT. We note that normally the vacuum expectation value of $n$ operators is defined as

$\left\langle O_{a}\left(w_{1}, \bar{w}_{1}\right) O_{a}\left(w_{2}, \bar{w}_{2}\right) \cdots O_{a}\left(w_{2 n}, \bar{w}_{2 n}\right)\right\rangle_{\Sigma_{n}} \equiv \frac{Z_{n}}{Z_{0 n}}$,

where $Z_{n}, Z_{0 n}$ are the partition functions with or without operators inserted on $\Sigma_{n}$. Following the replica method in the Euclidean path-integral formalism ${ }^{3}$ [35], we can express the reduced density as $\operatorname{Tr} \rho^{n}=Z_{n} / Z_{1}^{n}$. As a result $\Delta S_{A}^{(n)}$ can be written as

$$
\begin{aligned}
\Delta S_{A}^{(n)} & =\frac{1}{1-n}\left(\log \operatorname{Tr} \rho^{n}-\log \operatorname{Tr} \rho_{0}^{n}\right) \\
& =\frac{1}{1-n}\left(\log \frac{Z_{n}}{Z_{0 n}}-n \log \frac{Z_{1}}{Z_{01}}\right) .
\end{aligned}
$$

One can see that (16) follows when identity operator 1 belongs to the Hilbert space of the theory; however, in Liouville field theory, the $n$-point function is defined by the path integral and therefore it is not normalized:

$$
\left\langle V_{\alpha_{1}}\left(w_{1}, \bar{w}_{1}\right) V_{\alpha_{2}}\left(w_{2}, \bar{w}_{2}\right) \cdots V_{\alpha_{n}}\left(w_{2 n}, \bar{w}_{2 n}\right)\right\rangle_{\Sigma_{n}} \equiv Z_{n} .
$$

Because $\quad \alpha \in\{Q / 2+i p \mid p \in \mathbb{R}\} \bigcup\{Q>\alpha>0\}\} \quad$ in LFT and SFLT, one cannot take all $V$ 's to be the identity operator, i.e., analytically continue $\alpha=\frac{Q}{2}+i p$ to $p=i Q / 2$.

In other words, (16) applies to the case of Liouville field theory, which gives the Rényi entanglement entropy $S_{A}^{(n)}\left(\left|V_{\alpha}\right\rangle\right)$ :

$$
S_{A}^{(n)}\left[V_{\alpha}|0\rangle\right](t)=\frac{1}{1-n} \log \frac{\left\langle V_{\bar{\alpha}}^{\dagger}\left(w_{1}, \bar{w}_{1}\right) V_{\alpha}\left(w_{2}, \bar{w}_{2}\right) \ldots V_{\bar{\alpha}}^{\dagger}\left(w_{2 n-1}, \bar{w}_{2 n-1}\right) V_{\alpha}\left(w_{2 n}, \bar{w}_{2 n}\right)\right\rangle_{\Sigma_{n}}}{\left(\left\langle V_{\bar{\alpha}}^{\dagger}\left(w_{1}, \bar{w}_{1}\right) V_{\alpha}\left(w_{2}, \bar{w}_{2}\right)\right\rangle_{\Sigma_{1}}\right)^{n}}
$$

In the LFT and SLFT considered in this paper, the identity operator does not belong to the Hilbert space and the vacuum state [44] cannot be considered a good reference state $^{4}$ such as that in rational CFTs. Therefore, the $S_{A}^{(n)}(1|0\rangle)$ in Eq. (16) cannot be applied in LFT and SLFT. We can define the difference $\Delta S_{A}^{(n)}\left[V_{\alpha}|0\rangle, V_{\alpha_{r}}|0\rangle\right](t)$ between the two excited states. Here $V_{\alpha}|0\rangle$ and $V_{\alpha_{r}}|0\rangle$ are the target state and reference state, respectively. Alternatively, we calculate $S_{A}^{(n)}\left(O_{a}|0\rangle\right)$ in the early time and the late time limits and define the difference of REE between the two states,

$$
\begin{aligned}
\Delta S_{A}^{(n)}\left[V_{\alpha}|0\rangle, V_{\alpha_{r}}|0\rangle\right](t)= & S_{A}^{(n)}\left[V_{\alpha}(t)\right]|0\rangle(t) \\
& -S_{A}^{(n)}\left[V_{\alpha_{r}}(t)|0\rangle\right](t),
\end{aligned}
$$

to study time evolution. For later convenience, we divide the primary operators in LFT and SLFT are divided into two classes in terms of the Liouville momentum ${ }^{5}$ :

$$
\alpha \text { and } \alpha_{r} \in\left\{\begin{array}{l}
\{\alpha \mid \alpha=Q / 2+i p, p \in \mathbb{R}\} \bigcup\{\alpha \mid Q / 2>\operatorname{Re}(\alpha)>Q / 4\} \\
\bigcup\{\alpha \mid Q / 4>\operatorname{Re}(\alpha)>0\}, \\
\{\alpha \mid \operatorname{Re}(\alpha)=Q / 4, \operatorname{Im}(\alpha) \neq 0\} \text { Marginal case. }
\end{array}\right.
$$

\section{The second REE in $c=1$ free boson field theory}

To begin with the analysis of REE in special irrational CFTs, the compact free boson theory with generic radius is a simple irrational theory to see the time evolution of the second REE. ${ }^{6}$ Following [37], the second the REE is as follows:

$$
\left.\left.S_{2}=\frac{\pi c L}{2 \beta}-\log \left[2^{-2 c / 3}(z \bar{z})^{c / 12}\right](1-z)(1-\bar{z})\right]^{-c / 24} Z(\tau(z), \bar{\tau}(\bar{z}))\right]+\frac{c}{2} \log \frac{\beta}{2 \pi \epsilon},
$$

\footnotetext{
${ }^{3}$ The replica method for the local operator excited states in field theory has been explained explicitly in Sec. 2.2 of [35].

${ }^{4}$ The existence of the translation invariant normalizable vacuum is not self-consistent with the classical equation of motion of Liouville field theory, which has been shown in [44]. The nonexistence of the $S L(2, C)$ invariant vacuum in the spectrum has an important consequence: the identity operator does not belong to the whole Hilbert space; this means that the external Liouville momentum $\alpha$ cannot be vanishing.

${ }^{5}$ The main reason to choose reference and target states from the same class in LFT and SLFT is to calculate a well-defined quantity to restore the quasiparticle picture.

${ }^{6}$ The authors of [37] called it the memory effect of REE.
} 
where

$Z(\tau, \bar{\tau})=\operatorname{Tr} \exp \left[2 \pi i \tau\left(L_{0}-\frac{c}{24}\right)-2 \pi i \bar{\tau}\left(\bar{L}_{0}-\frac{c}{24}\right)\right]$.

The partition function can be calculated with the torus modulus, which is defined by

$$
z=\frac{\theta_{2}(\tau)^{4}}{\theta_{3}(\tau)^{4}}, \quad \tau=i \frac{K(1-z)}{K(z)} .
$$

Here, $K(z)=\frac{\pi}{2} 2 F_{1}\left(, \frac{1}{2}, 1, z^{2}\right)$.

For $z \rightarrow 0$, the relation is

$$
z=16 \sqrt{q}+O(q), \quad q \equiv e^{2 \pi i \tau} .
$$

For $z \rightarrow 1$, the relation is

$$
1-z=16 \sqrt{q^{\prime}}+O\left(q^{\prime}\right), \quad q^{\prime} \equiv e^{-2 \pi i / \tau} .
$$

We can analyze the two light cone singularities of the second Reyni entropy of this theory explicitly, via the torus partition function [30]

$$
Z(R)=\frac{1}{\eta(\tau) \bar{\eta}(\bar{\tau})} \sum_{e, m \in \mathbb{Z}} q^{h_{e, m}} \bar{q}^{\bar{h}_{e, m}},
$$

where $\eta$ denotes the Dedekind eta function. The conformal weights are as follows:

$h_{e, m}=\frac{1}{2}\left(\frac{e}{R}+\frac{m R}{2}\right)^{2} \quad \bar{h}_{e, m}=\frac{1}{2}\left(\frac{e}{R}-\frac{m R}{2}\right)^{2}$.

Here $e, m$ are integer numbers. When $R^{2}$ is irrational, there is no degeneracy in the weights and the theory is irrational CFT. In the early time limit $\tau \rightarrow i \infty, \bar{\tau} \rightarrow-i \infty, q \rightarrow 0$, $\bar{q} \rightarrow 0^{+}$, the partition function behaves as

$$
Z(\tau, \bar{\tau}) \sim q^{-c / 24} \bar{q}^{-c / 24} \sim 2^{2 c / 3} z^{-c / 12} \bar{z}^{-c / 12} .
$$

Then the second REE is as follows:

$$
S^{(2)}=\frac{\pi c L}{2 \beta}+\frac{c}{2} \log \frac{\beta}{2 \pi \epsilon} .
$$

In the late time limit $\tau \rightarrow i 0^{-}, \bar{\tau} \rightarrow-i \infty, q \rightarrow 1, \bar{q} \rightarrow 0^{+}$, the partition function behaves as

$$
Z(R)=\frac{1}{\eta(\tau) \bar{\eta}(\bar{\tau})}\left(1+q^{h_{\min }} \bar{q}^{\bar{h}_{\min }}+\cdots\right) .
$$

We have used the following properties:

$$
\eta(-1 / \tau)=\sqrt{-i \tau} \eta(\tau), \quad \bar{\eta}(\bar{\tau})=\sqrt{\frac{-i}{\bar{\tau}}} \bar{\eta}\left(\bar{\tau}^{\prime}\right) .
$$

Thus

$$
Z(R) \sim q^{-1 / 24} \bar{q}^{-1 / 24} .
$$

Then, the late time limit of the second REE in $c=1$ compact free boson theory is

$$
S^{(2)}=\frac{\pi c L}{2 \beta}-\log \left(2^{\frac{-2 c}{3}}\right)+\frac{c}{2} \log \frac{\beta}{2 \pi \epsilon} .
$$

The second term in (24) does not vanish in the late time limit.

Finally, the variation of the second REE between the early time and the late time is

$$
\Delta S^{(2)}=\frac{2}{3} \log 2 .
$$

Here we have chosen $c=1$.

The compact free boson theory on generic radius as "nearly rational" theory and the entanglement entropy has quasiparticle behavior, which is consistent with the criterion of a quasiparticle picture [37]. In the remaining parts of this paper, we will calculate REE in Liouville and superLiouville field theories, which have different Hilbert space structures. The first difference is that the vacuum state is not contained in the Hilbert space of LFT and SLFT and the second one is that the spectra in LFT and SLFT are continuous. Due to these two differences, the above calculations cannot be applied to LFT and SLFT directly. We will show later what will happen to REE in LFT and SLFT in more detail.

\section{E. The second REE in Liouville field theory}

We are mainly interested in the second REE here, which is associated with the four-point function in terms of Eq. (15). The four-point functions in LFT have been reviewed in Appendix A. In our setup, the interested $\left\langle V_{\bar{\alpha}} V_{\alpha} V_{\bar{\alpha}} V_{\alpha}\right\rangle_{\Sigma_{1}}$ is given by Eq. (A11). In LFT, the four-point Green function of primary operator $V_{\alpha}$ in the $s$-channel can be expressed by

$$
\begin{aligned}
\left\langle V_{\alpha_{1}} V_{\alpha_{2}} V_{\alpha_{3}} V_{\alpha_{4}}\right\rangle_{\Sigma_{1}} & =\left|z_{13}\right|^{-4 \Delta}\left|z_{24}\right|^{-4 \Delta} G_{1234}(z, \bar{z}) \\
& =\frac{1}{2}\left|z_{13}\right|^{-4 \Delta}\left|z_{24}\right|^{-4 \Delta} \int_{\mathbb{R}} \frac{d p}{2 \pi} C\left(\alpha_{1}, \alpha_{2}, \frac{Q}{2}+i p\right) C\left(\alpha_{3}, \alpha_{4}, \frac{Q}{2}-i p\right) F_{s}\left(\Delta_{i=1,2,3,4}, \Delta_{p}, z\right) F_{s}\left(\Delta_{i=1,2,3,4}, \Delta_{p}, \bar{z}\right) .
\end{aligned}
$$

The $\Delta_{i}=\alpha_{i}\left(Q-\alpha_{i}\right)$ is the conformal dimension of external Liouville momentum $\alpha_{i} \in\{\alpha \mid \alpha=Q / 2+i p, p \in \mathbb{R}\} \bigcup$ $\{\alpha \mid Q / 2>\operatorname{Re}(\alpha)>Q / 4\} \bigcup\{\alpha \mid Q / 4>\operatorname{Re}(\alpha)>0\}$. The integration over intermediate momentum $p$ stands for contour 
integration over $p \in \mathbb{R} . F_{s}\left(\Delta_{i=1,2,3,4}, \Delta_{p}, z\right)$ and $F_{s}\left(\Delta_{i=1,2,3,4}, \Delta_{p}, \bar{z}\right)$ are the holomorphic and antiholomorphic conformal blocks, respectively. The Dorn-Otto and Zamolodchikov-Zamolodchikov (DOZZ) formulas $C\left(\bar{\alpha}, \alpha, \frac{O}{2}+i p\right)$ are given in Appendix A 1. More precisely, for $\alpha_{i} \in(0, Q)$, the $G_{1234}(z, \bar{z})$ in the $s$-channel can be modified and expressed as follows:

$$
G_{1234}(z, \bar{z})=\sum_{\alpha_{s}^{\prime} \in D} D_{\alpha_{s}^{\prime}}(z, \bar{z})+\int \frac{d p}{2 \pi} C\left(\alpha_{1}, \alpha_{2}, \frac{Q}{2}+i p\right) C\left(\alpha_{3}, \alpha_{4}, \frac{Q}{2}-i p\right) F_{s}\left(\Delta_{i=1,2,3,4}, \Delta_{p}, z\right) F_{s}\left(\Delta_{i=1,2,3,4}, \Delta_{p}, \bar{z}\right),
$$

where $D$ denotes the discrete terms (A68) ${ }^{7}$ reviewed in Appendices A 5 and A 6 .

First, let us calculate the REE in the early time limit. One can make use of the $s$-channel expression in Eq. (A11) for $\left\langle V_{\bar{\alpha}} V_{\alpha} V_{\bar{\alpha}} V_{\alpha}\right\rangle$ :

$$
\begin{aligned}
G_{\bar{\alpha} \alpha \bar{\alpha} \alpha}(z, \bar{z}) & =\frac{1}{2} \int_{\mathbb{R}}^{\prime} \frac{d p}{2 \pi} C\left(\bar{\alpha}, \alpha, \frac{Q}{2}+i p\right) C\left(\bar{\alpha}, \alpha, \frac{Q}{2}-i p\right) F_{s}\left(\Delta_{i=\bar{\alpha}, \alpha, \bar{\alpha}, \alpha}, \Delta_{p}, z\right) F_{s}\left(\Delta_{i=\bar{\alpha}, \alpha, \bar{\alpha}, \alpha}, \Delta_{p}, \bar{z}\right) \\
& =\frac{1}{2} \int_{\mathbb{R}} \frac{d p}{2 \pi}\left[C\left(\bar{\alpha}, \alpha, \frac{Q}{2}+i p\right) C\left(\bar{\alpha}, \alpha, \frac{Q}{2}-i p\right) \bar{z}^{\Delta_{Q / 2+i p}-2 \Delta_{\alpha}}(1+\cdots) z^{\Delta_{Q / 2+i p}-2 \Delta_{\alpha}}(1+\cdots)\right] .
\end{aligned}
$$

We have shown the asymptotic behavior of the early time limit in the last step in (41).

Once we take the early time limit of Eq. (41), then

$$
\left.\left.\lim _{(z, \bar{z}) \rightarrow(0,0)}\left\langle V_{\bar{\alpha}} V_{\alpha} V_{\bar{\alpha}} V_{\alpha}\right\rangle_{\Sigma_{1}} \simeq\left|z_{13}\right|^{-4 \Delta}\left|z_{24}\right|^{-4 \Delta} \frac{d^{2} f_{\alpha}(p)}{d p^{2}}\right|_{p \rightarrow 0} \int_{\mathbb{R}}|z|^{-4 \Delta_{\alpha}+2 \Delta_{Q / 2+i p}} p^{2} d p \simeq \frac{\sqrt{\pi}}{8 \times 2 !} \frac{d^{2} f_{\alpha}(p)}{d p^{2}}\right|_{p \rightarrow 0}|z|^{-2\left(2 \Delta_{\alpha}-\Delta_{Q / 2}\right)} \ln ^{-\frac{3}{2}}|1 / z|,
$$

where we define

$$
f_{\alpha}(p)=C\left(\bar{\alpha}, \alpha, \frac{Q}{2}+i p\right) C\left(\bar{\alpha}, \alpha, \frac{Q}{2}-i p\right) .
$$

Here we have used the saddle point approximation presented in Appendix A 6 to obtain the leading behavior in the early time limit. The two-point Green function for the primary operator in LFT is as follows:

$$
\left\langle V_{\alpha}\left(x_{1}\right) V_{\alpha}\left(x_{2}\right)\right\rangle_{\Sigma_{1}}=\frac{S(\alpha) \delta(0)}{\left(x_{12} \bar{x}_{12}\right)^{2 \Delta_{\alpha_{1}}}}
$$

The $\delta(0)$ is proportional to the volume of the dilation group $\operatorname{Vol}($ dilaton $)=\int_{0}^{\infty} \frac{d \lambda}{\lambda}=\infty$. Then using the "reflection relationship" [45] $V_{\alpha}=S(\alpha) V_{Q-\alpha}$, one can obtain

$$
\left\langle V_{\bar{\alpha}}\left(x_{1}\right) V_{\alpha}\left(x_{2}\right)\right\rangle_{\Sigma_{1}}=\frac{\delta(0)}{\left(x_{12} \bar{x}_{12}\right)^{2 \Delta_{\alpha_{1}}}} .
$$

In terms of Eq. (15) and the early time limit, the ratio becomes

$$
\begin{aligned}
R_{\mathrm{EE}^{(2)}}^{(2, \bar{z}) \rightarrow(0,0)} \underset{(z, \bar{z}) \rightarrow(0,0)}{\simeq} \frac{\left\langle V_{\bar{\alpha}} V_{\alpha} V_{\bar{\alpha}} V_{\alpha}\right\rangle_{\Sigma_{2}}}{\left\langle V_{\bar{\alpha}} V_{\alpha}\right\rangle_{\Sigma_{1}}} \\
\left.\underset{(z, \bar{z}) \rightarrow(0,0)}{\simeq} \frac{\sqrt{\pi}}{8 \times 2 !} \frac{1}{\delta^{2}(0)} \frac{d^{2} f_{\alpha}(p)}{d p^{2}}\right|_{p \rightarrow 0}|z|^{2 \Delta_{Q / 2}} \ln ^{-\frac{3}{2}}|1 / z| .
\end{aligned}
$$

\footnotetext{
${ }^{7}$ In our setup of REE, the four-point Green function does not involve any discrete terms.
}

One can choose the appropriate normalization condition to remove the $\delta^{2}(0)$ dependence. $^{8}$

Then

$$
\begin{aligned}
S_{\mathrm{EE}}^{(2)} \underset{(z, \bar{z}) \rightarrow(0,0)}{\simeq} & -\log \left(R_{\mathrm{EE}}^{(2)}\right) \\
\underset{(z, \bar{z}) \rightarrow(0,0)}{\simeq} & -\log \left(\frac{\sqrt{\pi}}{8 \times 2 !} \frac{1}{\delta^{2}(0)}\right. \\
& \left.\times\left.\frac{d^{2} f_{\alpha}(p)}{d p^{2}}\right|_{p \rightarrow 0}|z|^{2 \Delta_{Q / 2}} \ln ^{-\frac{3}{2}}|1 / z|\right) .
\end{aligned}
$$

Since the second REE is associated with $\left\langle V_{\bar{\alpha}} V_{\alpha} V_{\bar{\alpha}} V_{\alpha}\right\rangle$, the identity operator cannot contribute to the intermediate channels. When the external Liouville momenta of the four-point function are $\alpha_{i} \in\{\alpha \mid \alpha=Q / 2+i p, p \in \mathbb{R}\} \bigcup$ $\{\alpha \mid Q / 2>\operatorname{Re}(\alpha)>Q / 4\} \bigcup\{\alpha \mid Q / 4>\operatorname{Re}(\alpha)>0\}$, the primary operators will not fuse into the identity operator. ${ }^{9}$ Therefore, the vacuum block in the intermediate channel will not contribute to the four-point function in the second

\footnotetext{
${ }^{8}$ Following standard regularization from Eq. (5.13) in [46], the normalization factor can absorb $\delta^{2}(0)$. Here, we just keep the factor $\delta^{2}(0)$ like in [38].

${ }^{9}$ Generally speaking, if $\operatorname{Re}\left(\alpha_{1}+\alpha_{2}\right)<Q / 2$, and there are discrete terms (A68) presented, then the identity operator will contribute to the four-point function as an intermediate channel operator. Since the second REE is associated with $\left\langle V_{\bar{\alpha}}(0) V_{\alpha}(z) V_{\bar{\alpha}}(1) V_{\alpha}(\infty)\right\rangle$ and $\operatorname{Re}\left(\alpha_{1}+\alpha_{2}\right) \geq Q / 2$, the discrete terms (52) will not contribute to the second REE.
} 
REE. To restore the quasiparticle picture [4,7] and make a well-defined quantity to show the memory effect of REE, we have to choose an appropriate reference state $V_{\alpha_{r}}|0\rangle$, which is not a vacuum state as given in (23).
In this paper, we choose a reference state which lives in the same class of target states and we can define the difference of the second REE in the early time limit as follows:

$$
\begin{aligned}
& \Delta S_{\mathrm{EE}}^{(2)}\left[V_{\alpha}|0\rangle, V_{\alpha_{r}}|0\rangle\right](t \rightarrow 0)=S_{\mathrm{EE}}^{(2)}\left[V_{\alpha}|0\rangle\right](t \rightarrow 0)-S_{\mathrm{EE}}^{(2)}\left[V_{\alpha_{r}}|0\rangle\right](t \rightarrow 0)=-\left.\log \left(\frac{f_{\alpha}^{\prime \prime}(p)}{f_{\alpha_{r}}{ }^{\prime \prime}(p)}\right)\right|_{p \rightarrow 0}, \\
& \alpha, \alpha_{r} \in\{\alpha \mid \alpha=Q / 2+i p, p \in \mathbb{R}\} \bigcup\{\alpha \mid Q / 2>\operatorname{Re}(\alpha)>Q / 4\} \bigcup\{\alpha \mid Q / 4>\operatorname{Re}(\alpha)>0\} .
\end{aligned}
$$

Here one can see that the $\Delta S_{\mathrm{EE}}^{(2)}\left[V_{\alpha}|0\rangle, V_{\alpha_{r}}|0\rangle\right](t \rightarrow 0)$ is finite. Explicitly, one can choose $\alpha_{r}=\alpha$ and the $\Delta S_{\mathrm{EE}}^{(2)}\left[V_{\alpha}|0\rangle, V_{\alpha_{r}}|0\rangle\right](t \rightarrow 0)$ will be vanishing, which shows that the quasiparticle picture has been restored. For $\alpha_{r} \neq \alpha$, $\Delta S_{\mathrm{EE}}^{(2)}\left[V_{\alpha}|0\rangle, V_{\alpha_{r}}|0\rangle\right](t \rightarrow 0)$ is finite with time evolution, which does not contradict the quasiparticle picture. When $\alpha, \alpha_{r}$ do not stay in the same class given by (23), the early and late time limits of $\Delta S_{\mathrm{EE}}^{(2)}\left[V_{\alpha}|0\rangle, V_{\alpha_{r}}|0\rangle\right]$ cannot be finite due to the different divergent powers of log divergence, e.g., (46) and (49). ${ }^{10}$

Generally speaking, for the four-point function $\left\langle V_{\alpha_{1}}(0) V_{\alpha_{2}}(z) V_{\alpha_{3}}(1) V_{\alpha_{4}}(\infty)\right\rangle_{\Sigma_{1}}$ with external legs $\alpha_{i}$ with $\operatorname{Re}\left(\alpha_{i}\right) \in(0, Q / 2)$, we have to consider the discrete terms' contributions which have been reviewed in Appendices A 5 and A 6. In our setup $\left\langle V_{\bar{\alpha}}(0) V_{\alpha}(z) V_{\bar{\alpha}}(1) V_{\alpha}(\infty)\right\rangle_{\Sigma_{1}}$, ${ }^{11}$ there is a marginal case: (i) $\operatorname{Re}(\alpha)=Q / 4, \operatorname{Im}(\alpha) \neq 0$ (marginal case).

Since the factor $C\left(\bar{\alpha}, \alpha, \alpha_{s}\right) C\left(\bar{\alpha}, \alpha, Q-\alpha_{s}\right)$ in Eq. (38) does not vanish at $\alpha_{s}=Q / 2$, we have

$$
\begin{aligned}
&\left\langle V_{\alpha}(0)\right.\left.V_{\alpha}(z) V_{\alpha}(1) V_{\alpha}(\infty)\right\rangle_{\Sigma_{1}} \\
& \underset{(z, \bar{z}) \rightarrow(0,0)}{\simeq} C\left(\bar{\alpha}, \alpha, \frac{Q}{2}\right)^{2} \int_{\mathbb{R}}|z|^{-2\left(2 \Delta_{\alpha}-\Delta 2 \alpha\right)-2 p^{2}} d p \\
& \underset{(z, \bar{z}) \rightarrow(0,0)}{\simeq} \frac{\sqrt{\pi}}{2} C\left(\bar{\alpha}, \alpha, \frac{Q}{2}\right)^{2}|z|^{-2\left(2 \Delta_{\alpha}-\Delta_{Q / 2}\right)} \ln ^{-\frac{1}{2}}|1 / z| .
\end{aligned}
$$

The ratio for the second REE in the early time limit reads

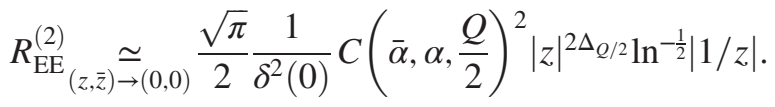

Then

$$
S_{\mathrm{EE}}^{(2)}\left[V_{\alpha}|0\rangle\right](t \rightarrow 0) \underset{(z, \bar{z}) \rightarrow(0,0)}{\simeq}-\log \left(\frac{\sqrt{\pi}}{2} \frac{1}{\delta^{2}(0)} C\left(\bar{\alpha}, \alpha, \frac{Q}{2}\right)^{2}|z|^{2 \Delta_{Q / 2}} \ln ^{-\frac{1}{2}}|1 / z|\right) .
$$

The second REE has the divergent factor $\ln ^{-\frac{1}{2}}|1 / z|$, which can be canceled by choosing the reference state $V_{\alpha_{r}}|0\rangle$, $\alpha_{r} \in\{a \in \mathbb{C} \mid \operatorname{Re}(a)=Q / 4, \operatorname{Im}(a) \neq 0\}$ to obtain a finite $\Delta S_{\mathrm{EE}}^{(2)}\left[V_{\alpha}|0\rangle, V_{\alpha_{r}}|0\rangle\right]$. In the early time limit, the difference of the second REE between the target states and the reference state is

$$
\begin{gathered}
\Delta S_{\mathrm{EE}}^{(2)}\left[V_{\alpha}|0\rangle, V_{\alpha_{r}}|0\rangle\right](t \rightarrow 0)=S_{\mathrm{EE}}^{(2)}\left[V_{\alpha}|0\rangle\right](t \rightarrow 0)-S_{\mathrm{EE}}^{(2)}\left[V_{\alpha_{r}}|0\rangle\right](t \rightarrow 0)=-\left.\log \left(\frac{f_{\alpha}(p)}{f_{\alpha_{r}}(p)}\right)\right|_{p \rightarrow 0}, \\
\alpha, \alpha_{r} \in\{a \in \mathbb{C} \mid \operatorname{Re}(a)=Q / 4, \operatorname{Im}(a) \neq 0\} .
\end{gathered}
$$

Now we will calculate the late time limit $(z, \bar{z}) \rightarrow(1,0)$ of the second REE. In this limit, applying the bootstrap equation to holomorphic conformal blocks will be convenient when extracting the correct late time behavior. The four-point function of primary fields in LFT can be expressed by holomorphic $t$-channel conformal blocks as given in Appendix A 1:

$$
\begin{aligned}
\left\langle V_{\bar{\alpha}} V_{\alpha} V_{\bar{\alpha}} V_{\alpha}\right\rangle_{\Sigma_{1}}= & \frac{1}{2}\left|z_{13}\right|^{-4 \Delta_{\alpha}}\left|z_{24}\right|^{-4 \Delta_{\alpha}} \int_{\mathbb{R}}^{\prime} \frac{d p}{2 \pi} C\left(\bar{\alpha}, \alpha, \frac{Q}{2}+i p\right) C\left(\bar{\alpha}, \alpha, \frac{Q}{2}-i p\right) \\
& \times F_{S}\left(\Delta_{i=\bar{\alpha}, \alpha, \bar{\alpha}, \alpha}, \Delta_{t}, \bar{z}\right) \int_{\mathbb{S}} d \alpha_{t} F_{\alpha_{s} \alpha_{t}}^{L}\left[\begin{array}{c}
\bar{\alpha} \alpha \\
\alpha \bar{\alpha}
\end{array}\right] F_{t}\left(\Delta_{i=\bar{\alpha}, \alpha, \bar{\alpha}, \alpha}, \Delta_{t}, z\right) .
\end{aligned}
$$

\footnotetext{
${ }^{10}$ In the remaining part of this paper, one can refer to the divergent piece of $S_{\mathrm{EE}}^{(2)}\left[V_{\alpha}|0\rangle\right]$ in the early and late time limits, respectively.

${ }^{11}$ Here we have chosen $\alpha_{1}=\bar{\alpha}=Q-\alpha, \alpha_{2}=\alpha, \operatorname{Re}\left(\alpha_{i}\right) \in(0, Q / 2)$; therefore, the discrete term will not be involved.
} 
The prime of integration over intermediate momentum $p$ stands for contour integration over reals with some additional so-called discrete terms' contributions. The integral in $\alpha_{t}$ is over $\mathbb{S}=\frac{Q}{2}+i \mathbb{R}^{+} . F_{\alpha_{s} \alpha_{t}}^{L}\left[\begin{array}{c}\bar{\alpha} \alpha \\ \alpha\end{array}\right]$ is the fusion matrix associated with the transformation from the $s$-channel to the $t$-channel and it has been revisited in Appendix A 7. More precisely, the $G_{\bar{\alpha}, \alpha, \bar{\alpha}, \alpha}(z, \bar{z})$ can be expressed by the holomorphic $t$-channel conformal block in terms of the conformal bootstrap equation as follows:

$$
\begin{aligned}
G_{\bar{\alpha}, \alpha, \bar{\alpha}, \alpha}(z, \bar{z})= & \sum_{\alpha_{s}^{\prime} \in D} \tilde{D}_{\alpha_{s}^{\prime}}(z, \bar{z})+\int_{\mathbb{R}^{+}} \frac{d p}{2 \pi} C\left(\bar{\alpha}, \alpha, \frac{Q}{2}+i p\right) C\left(\bar{\alpha}, \alpha, \frac{Q}{2}-i p\right) \\
& \times F_{s}\left(\Delta_{i=\bar{\alpha}, \alpha, \bar{\alpha}, \alpha}, \Delta_{t}, \bar{z}\right) \int_{\mathbb{S}} d \alpha_{t} F_{\alpha_{s} \alpha_{t}}^{L}\left[\begin{array}{c}
\bar{\alpha} \alpha \\
\alpha \bar{\alpha}
\end{array}\right] F_{t}\left(\Delta_{i=\bar{\alpha}, \alpha, \bar{\alpha}, \alpha}, \Delta_{t}, z\right),
\end{aligned}
$$

where $\tilde{D}$ is the finite set of discrete terms that have been reviewed in Appendices A 5 and A 6 . The $D$ is the set of double poles ${ }^{12}$ induced by the factors $C\left(\bar{\alpha}, \alpha, \alpha_{s}\right) C\left(\bar{\alpha}, \alpha, \bar{\alpha}_{s}\right)$ and $\tilde{D}_{\alpha_{s}^{\prime}}(z, \bar{z})$ is given by the last line in Eq. (A60).

In the late time limit, the leading contributions to the REE will be as follows:

$$
\begin{aligned}
\left\langle V_{\bar{\alpha}} V_{\alpha} V_{\bar{\alpha}} V_{\alpha}\right\rangle_{\Sigma_{1}} \underset{(z, \bar{z}) \rightarrow(1,0)}{\simeq} \frac{1}{2}\left|z_{13}\right|^{-4 \Delta}\left|z_{24}\right|^{-4 \Delta} \int_{\mathbb{R}}^{\prime} \frac{d p}{2 \pi} C\left(\bar{\alpha}, \alpha, \frac{Q}{2}+i p\right) C\left(\bar{\alpha}, \alpha, \frac{Q}{2}-i p\right) \bar{z}^{\Delta_{p}-2 \Delta} \int_{\mathbb{S}} d \alpha_{t} F_{\alpha_{s} \alpha_{t}}^{L}\left[\begin{array}{l}
\bar{\alpha} \alpha \\
\alpha \bar{\alpha}
\end{array}\right](1-z)^{\Delta_{t}-2 \Delta} \\
\underset{(z, \bar{z}) \rightarrow(1,0)}{\simeq} \frac{1}{2}\left|z_{13}\right|^{-4 \Delta}\left|z_{24}\right|^{-4 \Delta} \int_{\mathbb{R}} \frac{d p}{2 \pi} C\left(\bar{\alpha}, \alpha, \frac{Q}{2}+i p\right) C\left(\bar{\alpha}, \alpha, \frac{Q}{2}-i p\right) \\
\quad \times \bar{z}^{\Delta_{p}-2 \Delta} \int_{\mathbb{S}} d \alpha_{t} F_{\alpha_{s} \alpha_{t}}^{L}\left[\begin{array}{c}
\bar{\alpha} \alpha \\
\alpha \bar{\alpha}
\end{array}\right](1-z)^{\Delta_{t}-2 \Delta}+\left|z_{13}\right|^{-4 \Delta}\left|z_{24}\right|^{-4 \Delta} \sum_{\alpha_{s}^{\prime} \in D} \tilde{D}_{\alpha_{s}^{\prime}}(z, \bar{z})
\end{aligned}
$$

For the external Liouville momentum $\alpha \in\{\alpha \mid \alpha=Q / 2+i p, p \in \mathbb{R}\} \bigcup\{\alpha \mid Q / 2>\operatorname{Re}(\alpha)>Q / 4\} \bigcup\{\alpha \mid Q / 4>\operatorname{Re}(\alpha)>0\}$ and $p \neq 0$, we take the late time limit of Eqs. (53) and (15) will be

$$
\begin{aligned}
& \left.\lim _{(z, \bar{z}) \rightarrow(1,0)}\left\langle V_{\bar{\alpha}} V_{\alpha} V_{\bar{\alpha}} V_{\alpha}\right\rangle_{\Sigma_{1}} \underset{(z, \bar{z}) \rightarrow(1,0)}{\simeq} \frac{\pi}{64 \times 2 ! \sqrt{\pi}} \frac{d^{2} f_{\alpha}\left(p_{s}\right)}{d p_{s}^{2}}\right|_{p_{s} \rightarrow 0} 2\left(s_{b}^{\prime}(Q)\right)^{2} \frac{F_{Q / 2, Q / 2}^{L}\left[\begin{array}{c}
\bar{\alpha} \alpha \\
\alpha \\
\alpha
\end{array}\right]}{\left|s_{b}(Q)\right|^{2}} \\
& \times(1-z)^{\Delta_{Q / 2}-2 \Delta_{\alpha}} \bar{z}^{\Delta_{Q / 2}-2 \Delta_{\alpha}} \ln ^{-3 / 2}\left(\frac{1}{(1-z)}\right) \ln ^{-3 / 2}\left(\frac{1}{\bar{z}}\right) .
\end{aligned}
$$

We have used the late time limit $(z, \bar{z}) \rightarrow(1,0)$ and saddle point approximation to extract the leading contribution from the relevant terms $\alpha_{s}=Q / 2, \alpha_{t}=Q / 2$.

In this limit, the ratio becomes

$$
\begin{aligned}
& R_{\mathrm{EE}}^{(2)} \underset{(z, \bar{z}) \rightarrow(1,0)}{\simeq} \lim _{(z, \bar{z}) \rightarrow(1,0)} \frac{\left\langle V_{\bar{\alpha}} V_{\alpha} V_{\bar{\alpha}} V_{\alpha}\right\rangle_{\Sigma_{2}}}{\left\langle V_{\bar{\alpha}} V_{\alpha}\right\rangle_{\Sigma_{1}}^{2}}
\end{aligned}
$$

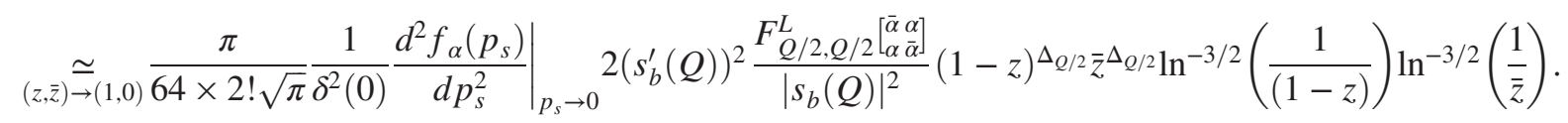

We use same normalization for the two-point Green function given in Eq. (45).

Then the second REE in the late time limit reads

$$
\begin{aligned}
& S_{\mathrm{EE}}^{(2)}\left[V_{\alpha}|0\rangle\right](t \rightarrow \infty) \underset{(z, \bar{z}) \rightarrow(1,0)}{\simeq}-\log \left(\left.\frac{\pi}{64 \times 2 ! \sqrt{\pi}} \frac{1}{\delta^{2}(0)} \frac{d^{2} f_{\alpha}(p)}{d p^{2}}\right|_{p \rightarrow 0} 2\left(s_{b}^{\prime}(Q)\right)^{2} \frac{\left.\left.F_{Q / 2, Q / 2}^{L}\right|_{\alpha} ^{\bar{\alpha} \alpha}\right]}{\left|s_{b}(Q)\right|^{2}}\right. \\
& \left.\times(1-z)^{\Delta_{Q / 2}} \bar{z}^{\Delta_{Q / 2}} \ln ^{-3 / 2}\left(\frac{1}{(1-z)}\right) \ln ^{-3 / 2}\left(\frac{1}{\bar{z}}\right)\right) .
\end{aligned}
$$

In the late time limit, the difference of the second REE between the target state and the reference state is

\footnotetext{
${ }^{12}$ For external Liouville momentum $\alpha \in\{\alpha \mid \alpha=Q / 2+i p, p \in \mathbb{R}\} \bigcup\{\alpha \mid Q / 2>\operatorname{Re}(\alpha)>Q / 4\} \bigcup\{\alpha \mid Q / 4>\operatorname{Re}(\alpha)>0\}$, there are no discrete terms.
} 


$$
\begin{aligned}
& \Delta S_{\mathrm{EE}}^{(2)}\left[V_{\alpha}|0\rangle, V_{\alpha_{r}}|0\rangle\right](t \rightarrow \infty)=S_{\mathrm{EE}}^{(2)}\left[V_{\alpha}|0\rangle\right](t \rightarrow \infty)-S_{\mathrm{EE}}^{(2)}\left[V_{\alpha_{r}}|0\rangle\right](t \rightarrow \infty)=-\log \left(\frac{f_{\alpha}^{\prime \prime}(p) F_{Q / 2, Q / 2}^{L}\left[\begin{array}{c}
\bar{\alpha} \alpha \\
\alpha \bar{\alpha}
\end{array}\right]}{\left.f_{\alpha_{r}}^{\prime \prime}(p) F_{Q / 2, Q / 2}^{L}\left[\begin{array}{c}
\bar{\alpha}_{r} \alpha_{r} \\
\alpha_{r}
\end{array}\right)\right|_{p \rightarrow 0} ^{\overline{\alpha_{r}}}}\right), \\
& \alpha, \alpha_{r} \in\{\alpha \mid \alpha=Q / 2+i p, p \in \mathbb{R}\} \bigcup\{\alpha \mid Q / 2>\operatorname{Re}(\alpha)>Q / 4\} \bigcup\{\alpha \mid Q / 4>\operatorname{Re}(\alpha)>0\} .
\end{aligned}
$$

Additionally, we have to consider the marginal case:

(i) $\operatorname{Re}(\alpha)=Q / 4, \operatorname{Im}(\alpha) \neq 0$ (marginal case).

Since $C\left(\bar{\alpha}, \alpha, \alpha_{s}\right) C\left(\bar{\alpha}, \alpha, Q-\alpha_{s}\right)$ does not vanish at $\alpha_{s}=Q / 2$, the late time of the four-point function behaves as follows:

$$
\begin{aligned}
& \left\langle V_{\bar{\alpha}}(0) V_{\alpha}(z) V_{\bar{\alpha}}(1) V_{\alpha}(\infty)\right\rangle_{\Sigma_{1}} \\
& \underset{(z, \bar{z}) \rightarrow(1,0)}{\simeq} C\left(\bar{\alpha}, \alpha, \frac{Q}{2}\right)^{2} \int_{\mathbb{R}} F_{\alpha_{s}=Q / 2+i p_{s}, \alpha_{t}=Q / 2+i p_{t}}^{L}\left[\begin{array}{c}
\alpha \alpha \\
\alpha \alpha
\end{array}\right](1-z)^{\Delta_{2 \alpha}-2 \Delta_{\alpha}+p_{s}^{2}}(\bar{z})^{\Delta_{2 \alpha}-2 \Delta_{\alpha}+p_{t}^{2}} d p_{s} d p_{t}
\end{aligned}
$$

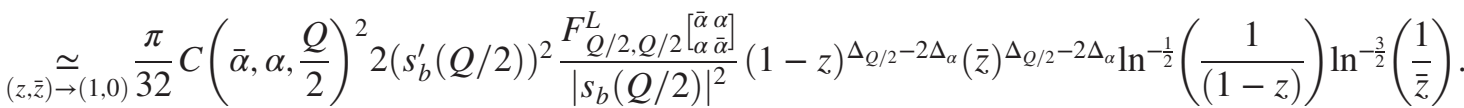

In the late time limit, the ratio for the second REE is

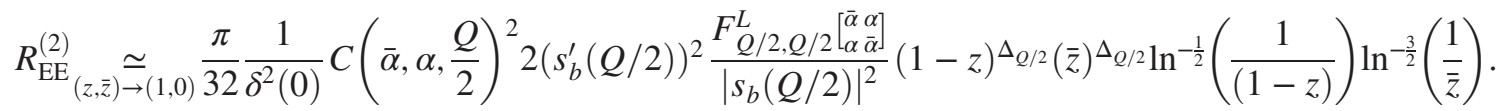

Then

$$
\begin{aligned}
& S_{\mathrm{EE}}^{(2)}\left[V_{\alpha}|0\rangle\right](t \rightarrow \infty)
\end{aligned}
$$

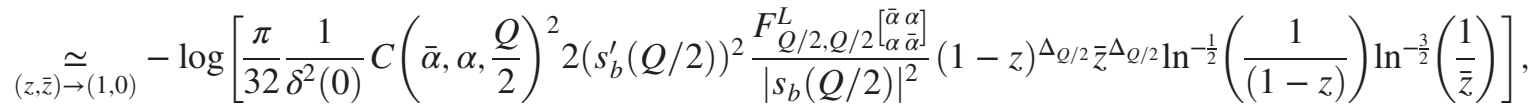

and the difference of the second REE between the target state and the reference state in the late time limit is

$$
\begin{gathered}
\Delta S_{\mathrm{EE}}^{(2)}\left[V_{\alpha}|0\rangle, V_{\alpha_{r}}|0\rangle\right](t \rightarrow \infty)=S_{\mathrm{EE}}^{(2)}\left[V_{\alpha}|0\rangle\right](t \rightarrow \infty)-S_{\mathrm{EE}}^{(2)}\left[V_{\alpha_{r}}|0\rangle\right](t \rightarrow \infty)=-\log \left(\frac{f_{\alpha}(p) F_{Q / 2, Q / 2}^{L}\left[\begin{array}{c}
\bar{\alpha} \alpha \\
\alpha \bar{\alpha}
\end{array}\right]}{f_{\alpha_{r}}(p) F_{Q / 2, Q / 2}^{L}\left[\left.\left._{\left.\bar{\alpha}_{r} \alpha_{r}\right]}^{L}\right|_{\alpha_{r} \bar{\alpha}_{r}} ^{L}\right|_{p \rightarrow 0}\right.}\right. \\
\alpha, \alpha_{r} \in\{a \in \mathbb{C} \mid \operatorname{Re}(a)=Q / 4, \operatorname{Im}(a) \neq 0\}
\end{gathered}
$$

\section{F. The second REE in super-Liouville field theory}

In this section, we would like to consider the states excited by local operators in super-Liouville field theory. For the sake of consistent notation, we review the corresponding contents of SLFT in Appendix A 2. The four-point Green function for the André Neveu and John Henry Schwarz (NS-NS) operator $V_{\bar{\alpha}}, V_{\alpha}$ with the $s$-channel intermediate states given in Eq. (A28) is as follows:

$$
\begin{aligned}
\left\langle V_{\bar{\alpha}} V_{\alpha} V_{\bar{\alpha}} V_{\alpha}\right\rangle_{\Sigma_{1}}= & \left|z_{13}\right|^{-4 \Delta}\left|z_{24}\right|^{-4 \Delta} G_{\overline{1} 2 \overline{3} 4}(z, \bar{z}) \\
= & \left|z_{13}\right|^{-4 \Delta}\left|z_{24}\right|^{-4 \Delta}\left[\int_{S}^{\prime} d \alpha_{S} C_{\mathrm{NS}}\left(\bar{\alpha}, \alpha, \alpha_{s}\right) C_{\mathrm{NS}}\left(\bar{\alpha}, \alpha, \bar{\alpha}_{S}\right) F_{S}^{e}\left(\Delta_{i=1,2,3,4}, \Delta_{p}, \bar{z}\right) F_{S}^{e}\left(\Delta_{i=1,2,3,4}, \Delta_{p}, z\right)\right. \\
& \left.+\int_{S}^{\prime} d \alpha_{S} \tilde{C}_{\mathrm{NS}}\left(\bar{\alpha}, \alpha, \alpha_{S}\right) \tilde{C}_{\mathrm{NS}}\left(\bar{\alpha}, \alpha, \alpha_{S}\right) F_{S}^{o}\left(\Delta_{i=1,2,3,4}, \Delta_{p}, \bar{z}\right) F_{S}^{o}\left(\Delta_{i=1,2,3,4}, \Delta_{p}, z\right)\right]
\end{aligned}
$$

The four-point green function for the Pierre Ramond (R-R) operator $R_{\bar{\alpha}}, R_{\alpha}$ reads similarly:

$$
\begin{aligned}
\left\langle R_{\bar{\alpha}} R_{\alpha} R_{\bar{\alpha}} R_{\alpha}\right\rangle_{\Sigma_{1}}= & \left|z_{13}\right|^{-4 \Delta}\left|z_{24}\right|^{-4 \Delta} G_{\overline{1} 2 \overline{3} 4}(z, \bar{z}) \\
= & \left|z_{13}\right|^{-4 \Delta}\left|z_{24}\right|^{-4 \Delta}\left[\int_{S}^{\prime} d \alpha_{S} C_{R}\left(\bar{\alpha}, \alpha, \alpha_{S}\right) C_{R}\left(\bar{\alpha}, \alpha, \bar{\alpha}_{S}\right) F_{S}^{e}\left(\Delta_{i=\bar{\alpha}, \alpha, \bar{\alpha}, \alpha}, \Delta_{p}, \bar{z}\right) F_{S}^{e}\left(\Delta_{i=\bar{\alpha}, \alpha, \bar{\alpha}, \alpha}, \Delta_{p}, z\right)\right. \\
& \left.+\int_{S}^{\prime} d \alpha_{s} \tilde{C}_{R}\left(\bar{\alpha}, \alpha, \alpha_{s}\right) \tilde{C}_{R}\left(\bar{\alpha}, \alpha, \alpha_{S}\right) F_{S}^{o}\left(\Delta_{i=\bar{\alpha}, \alpha, \bar{\alpha}, \alpha}, \Delta_{p}, \bar{z}\right) F_{s}^{o}\left(\Delta_{i=\bar{\alpha}, \alpha, \bar{\alpha}, \alpha}, \Delta_{p}, z\right)\right] .
\end{aligned}
$$


All the calculations of REE for the NS-NS states can be directly generalized to states excited by the R-R operators and hence we only carry out the analysis in the former case (62).

We start with the external super-Liouville momentum $\alpha_{i}=\{\alpha \mid \alpha=Q / 2+i p, p \in \mathbb{R}\} \bigcup\{\alpha \mid Q / 2>\operatorname{Re}(\alpha)>$ $Q / 4\} \bigcup\{\alpha \mid Q / 4>\operatorname{Re}(\alpha)>0\}$. With saddle point approximation, the early time behavior of Eq. (62) is as follows:

$$
\begin{aligned}
\left.\lim _{(z, \bar{z}) \rightarrow(0,0)}\left\langle V_{\bar{\alpha}} V_{\alpha} V_{\bar{\alpha}} V_{\alpha}\right\rangle_{\Sigma_{1}} \underset{(z, \bar{z}) \rightarrow(0,0)}{\stackrel{\sim}{\simeq}} \frac{1}{2 !} \frac{d^{2} f_{\alpha \mathrm{NS}}(p)}{d p^{2}}\right|_{p \rightarrow 0} \int_{\mathbb{R}}|z|^{-2\left(2 \Delta_{\alpha}-\Delta_{Q / 2}\right)+2 p^{2}} p^{2} d p \\
\left.\underset{(z, \bar{z}) \rightarrow(0,0)}{\simeq} \frac{\sqrt{\pi}}{4 \times 2 !} \frac{d^{2} f_{\alpha \mathrm{NS}}(p)}{d p^{2}}\right|_{p \rightarrow 0}|z|^{-2\left(2 \Delta_{\alpha}-\Delta_{Q / 2}\right)} \ln ^{-\frac{3}{2}}|1 / z|,
\end{aligned}
$$

where $\quad f_{\alpha \mathrm{NS}}\left(p_{s}\right)=C_{\mathrm{NS}}\left(\bar{\alpha}, \alpha, \alpha_{s}\right) C_{\mathrm{NS}}\left(\bar{\alpha}, \alpha, \bar{\alpha}_{s}\right) \quad$ and $\tilde{f}_{\alpha \mathrm{NS}}=\tilde{C}_{\mathrm{NS}}\left(\bar{\alpha}, \alpha, \alpha_{s}\right) \tilde{C}_{\mathrm{NS}}\left(\bar{\alpha}, \alpha, \alpha_{s}\right)$. In the early time limit $(z, \bar{z}) \rightarrow(0,0)$, we have used the fact that the leading intermediate state in the parity odd conformal block $F_{s}^{o}\left(\Delta_{i=\bar{\alpha}, \alpha, \bar{\alpha}, \alpha}, \Delta_{p}, \bar{z}\right) F_{s}^{o}\left(\Delta_{i=\bar{\alpha}, \alpha, \bar{\alpha}, \alpha}, \Delta_{p}, z\right) \quad$ in $\quad(62)$ is $G_{-1 / 2} \tilde{G}_{-1 / 2} V_{\alpha_{s}}$ and hence its contribution will be smaller by a factor of $z^{1 / 2}$ compared to the even conformal block (see [47] for more details). As a result we can make the contribution from the parity-even conformal block [48]. For the R-R sector, we directly replace $C_{\mathrm{NS}}\left(\alpha_{1}, \alpha_{2}, \alpha_{3}\right)$ with $C_{R}\left(\alpha_{1}, \alpha_{2}, \alpha_{3}\right)$. The structure constant $C_{R}\left(\alpha_{1}, \alpha_{2}, \alpha_{s}\right)$ has the simple pole at $\alpha_{s}=Q / 2$ as $C_{\mathrm{NS}}\left(\alpha_{1}, \alpha_{2}, \alpha_{s}\right)$, which is from $\Upsilon_{\mathrm{NS}}\left(\alpha_{3}\right)$ in the numerator of (A23) and (A29); therefore, the analysis of the local excited states associated with the R-R operator is the same as those with a NS-NS operator.
The two-point Green function for the primary operator in the NS sector is as follows:

$$
\left\langle V_{\alpha}\left(x_{1}\right) V_{\alpha}\left(x_{2}\right)\right\rangle_{\Sigma_{1}}=\frac{D_{\mathrm{NS}}(\alpha) \delta(0)}{\left(x_{12} \bar{x}_{12}\right)^{2 \Delta_{\alpha}}}
$$

with

$$
D_{\mathrm{NS}}(\alpha)=\left(\pi \mu \gamma\left(\frac{b Q}{2}\right)\right)^{\frac{(Q-2 \alpha)}{b}} \frac{b^{2} \gamma\left(b \alpha-\frac{1}{2}-\frac{b^{2}}{2}\right)}{\gamma\left(\frac{1}{2}+\frac{b^{-2}}{2}-\alpha b^{-1}\right)} .
$$

Then using the "reflection relationship" $V_{\alpha}=D_{\mathrm{NS}}(\alpha) V_{Q-\alpha}$, one can obtain

$$
\left\langle V_{\bar{\alpha}}\left(x_{1}\right) V_{\alpha}\left(x_{2}\right)\right\rangle_{\Sigma_{1}}=\frac{\delta(0)}{\left(x_{12} \bar{x}_{12}\right)^{2 \Delta_{\alpha}}} .
$$

The associated ratio in the early time limit is

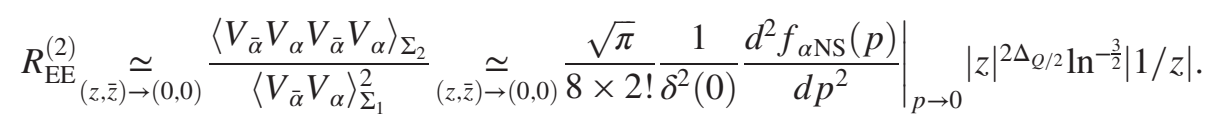

As we have done in LFT, we also keep the normalization factor with a delta function in explicit form.

Then

$$
S_{\mathrm{EE}}^{(2)}(t \rightarrow 0)=-\log \left(R_{\mathrm{EE}}^{(2)}\right) \underset{(z, \bar{z}) \rightarrow(0,0)}{\simeq}-\log \left(\left.\frac{\sqrt{\pi}}{8 \times 2 !} \frac{1}{\delta^{2}(0)} \frac{d^{2} f_{\alpha \mathrm{NS}}(p)}{d p^{2}}\right|_{p \rightarrow 0}|z|^{2 \Delta_{Q / 2}} \ln ^{-\frac{3}{2}}|1 / z|\right) .
$$

Finally, the early time of the difference of the second REE between $V_{\alpha}|0\rangle$ and $V_{\alpha_{r}}|0\rangle$ is

$$
\begin{aligned}
& \Delta S_{\mathrm{EE}}^{(2)}\left[V_{\alpha}|0\rangle, V_{\alpha_{r}}|0\rangle\right](t \rightarrow 0)=S_{\mathrm{EE}}^{(2)}\left[V_{\alpha}|0\rangle\right](t \rightarrow 0)-S_{\mathrm{EE}}^{(2)}\left[V_{\alpha_{r}}|0\rangle\right](t \rightarrow 0)=-\left.\log \left(\frac{f_{\alpha \mathrm{NS}}{ }^{\prime \prime}(p)}{f_{\alpha_{r} \mathrm{NS}}{ }^{\prime \prime}(p)}\right)\right|_{p \rightarrow 0}, \\
& \alpha, \alpha_{r} \in\{\alpha \mid \alpha=Q / 2+i p, p \in \mathbb{R}\} \bigcup\{\alpha \mid Q / 2>\operatorname{Re}(\alpha)>Q / 4\} \bigcup\{\alpha \mid Q / 4>\operatorname{Re}(\alpha)>0\} .
\end{aligned}
$$

Additionally, we have to consider the marginal case:

(i) $\operatorname{Re}(\alpha)=\frac{Q}{4}, \operatorname{Im}(\alpha) \neq 0$ (marginal case).

This case is similar to that mentioned above, except that $C_{\mathrm{NS}}\left(\bar{\alpha}, \alpha, \alpha_{s},\right) C_{\mathrm{NS}}\left(\bar{\alpha}, \alpha, Q-a_{s}\right)$ does not vanish at $\alpha_{s}=Q / 2$, so we have 


$$
\begin{aligned}
\left\langle V_{\bar{\alpha}}(0) V_{\alpha}(z) V_{\bar{\alpha}}(1) V_{\alpha}(\infty)\right\rangle_{\Sigma_{1}} \underset{(z, \bar{z}) \rightarrow(0,0)}{\simeq} C_{\mathrm{NS}}\left(\bar{\alpha}, \alpha, \frac{Q}{2}\right)^{2} \int_{R}|z|^{-2\left(2 \Delta_{2 \alpha}-\Delta_{\alpha}\right)+2 p^{2}} d p \\
\underset{(z, \bar{z}) \rightarrow(0,0)}{\simeq} \frac{\sqrt{\pi}}{2} C_{\mathrm{NS}}\left(\bar{\alpha}, \alpha, \frac{Q}{2}\right)^{2}|z|^{-2\left(\Delta_{\alpha}-\Delta_{Q / 2}\right)} \ln ^{-\frac{1}{2}}|1 / z| .
\end{aligned}
$$

The ratio for the second REE in the early time limit is

$$
R_{\mathrm{EE}}^{(2)} \underset{(z, \bar{z}) \rightarrow(0,0)}{\simeq} \frac{\sqrt{\pi}}{2} \frac{1}{\delta^{2}(0)} C_{\mathrm{NS}}\left(\bar{\alpha}, \alpha, \frac{Q}{2}\right)^{2}|z|^{2 \Delta_{Q / 2}} \ln ^{-\frac{1}{2}}|1 / z| .
$$

Then

$$
S_{\mathrm{EE}}^{(2)}\left[V_{\alpha}|0\rangle\right](t \rightarrow 0) \underset{(z, \bar{z}) \rightarrow(0,0)}{\simeq}-\log \left(\frac{\sqrt{\pi}}{2} \frac{1}{\delta^{2}(0)} C_{\mathrm{NS}}\left(\bar{\alpha}, \alpha, \frac{Q}{2}\right)^{2}|z|^{2 \Delta_{Q / 2}} \ln ^{-\frac{1}{2}}|1 / z|\right) .
$$

The second REE has a divergent factor $\ln ^{-\frac{1}{2}}|1 / z|$, which can be canceled by choosing the reference state $V_{\alpha_{r}}|0\rangle$, $\alpha_{r} \in\{a \in \mathbb{C} \mid \operatorname{Re}(a)=Q / 4, \operatorname{Im}(a) \in \mathbb{R}, \operatorname{Im}(\alpha) \neq 0\}$. Finally, the early time of the difference of the second REE between $V_{\alpha}|0\rangle$ and $V_{\alpha_{r}}|0\rangle$ is

$$
\begin{aligned}
& \Delta S_{\mathrm{EE}}^{(2)}\left[V_{\alpha}|0\rangle, V_{\alpha_{r}}|0\rangle\right](t \rightarrow 0)=S_{\mathrm{EE}}^{(2)}\left[V_{\alpha}|0\rangle\right](t \rightarrow 0)-S_{\mathrm{EE}}^{(2)}\left[V_{\alpha_{r}}|0\rangle\right](t \rightarrow 0)=-\log \left(\frac{C_{\mathrm{NS}}\left(\bar{\alpha}, \alpha, \frac{Q}{2}\right)^{2}}{C_{\mathrm{NS}}\left(\bar{\alpha}_{r}, \alpha_{r}, \frac{Q}{2}\right)^{2}}\right) \\
& \alpha, \alpha_{r} \in\{a \in \mathbb{C} \mid \operatorname{Re}(a)=Q / 4, \operatorname{Im}(a) \in \mathbb{R}, \operatorname{Im}(\alpha) \neq 0\} .
\end{aligned}
$$

Secondly, we consider the second REE in SLFT in the late time limit. For convenience in the late time limit, we have to use a conformal bootstrap equation to express the four-point function $G_{1234}(z, \bar{z})$, which is similar to the procedures shown in LFT. The four-point function can be expressed as follows:

$$
\begin{aligned}
\left\langle V_{\alpha_{1}}(0,0) V_{\alpha_{2}}(z, \bar{z}) V_{\alpha_{3}}(1,1) V_{\alpha_{4}}(\infty, \infty)\right\rangle_{\Sigma_{1}}= & G_{1234}(z, \bar{z}) \\
= & \frac{1}{2}\left(\int_{S}^{\prime} d \alpha_{s} C_{\mathrm{NS}}\left(\alpha_{1}, \alpha_{2}, \alpha_{s}\right) C_{\mathrm{NS}}\left(\alpha_{3}, \alpha_{4}, \bar{\alpha}_{s}\right) F_{s}^{e}\left(\Delta_{i=1,2,3,4}, \Delta_{\alpha_{s}}, \bar{z}\right)\right. \\
& \times \int d \alpha_{t} \sum_{\rho=e, o} F_{\alpha_{s} \alpha_{t}}^{S L}\left[\begin{array}{c}
\bar{\alpha} \alpha \\
\alpha \bar{\alpha}
\end{array}\right]_{\rho}^{e} F_{t}^{\rho}\left(\Delta_{i=1,2,3,4}, \Delta_{\alpha_{t}}, z\right) \\
& +\int_{S}^{\prime} d \alpha_{s} \tilde{C}_{\mathrm{NS}}\left(\alpha_{1}, \alpha_{2}, \alpha_{s}\right) \tilde{C}_{\mathrm{NS}}\left(\alpha_{3}, \alpha_{4}, \bar{\alpha}_{s}\right) F_{s}^{o}\left(\Delta_{i=1,2,3,4}, \Delta_{\alpha_{s}}, \bar{z}\right) \\
& \left.\times \int d \alpha_{t} \sum_{\rho=e, o} F_{\alpha_{s} \alpha_{t}}^{S L}\left[\begin{array}{c}
\alpha_{3} \alpha_{2} \\
\alpha_{4} \alpha_{1}
\end{array}\right]_{\rho}^{o} F_{t}^{\rho}\left(\Delta_{i=1,2,3,4}, \Delta_{\alpha_{t}}, z\right)\right) .
\end{aligned}
$$

In the late time limit of Eq. (73) with saddle point approximation, the four-point function becomes

$$
\begin{aligned}
& \lim _{(z, \bar{z}) \rightarrow(1,0)}\left\langle V_{\bar{\alpha}} V_{\alpha} V_{\bar{\alpha}} V_{\alpha}\right\rangle_{\Sigma_{1}} \\
& \left.\underset{(z, \bar{z}) \rightarrow(1,0)}{\simeq} \frac{1}{2 !} \frac{d^{2} f_{\alpha \mathrm{NS}}\left(p_{s}\right)}{d p_{s}^{2}}\right|_{p_{s} \rightarrow 0} \int_{\mathbb{R}} F_{\alpha_{s}=Q / 2+i p_{s}, \alpha_{t}=Q / 2+i p_{t}}^{S L}\left[\begin{array}{c}
\bar{\alpha} \alpha \\
\alpha \bar{\alpha}
\end{array}\right]_{e}^{e}(1-z)^{\Delta_{Q / 2}-2 \Delta_{\alpha}+p^{2}}(\bar{z})^{\Delta_{Q / 2}-2 \Delta_{\alpha}+p_{s}^{2}} p_{s}^{2} d p_{s} d p_{t} . \\
& \left.\underset{(z, \bar{z}) \rightarrow(1,0)}{\simeq} \frac{\pi}{64 \times 2 !} \frac{d^{2} f_{\alpha \mathrm{NS}}\left(p_{s}\right)}{d p_{s}^{2}}\right|_{p_{s} \rightarrow 0} 2\left(s_{\mathrm{NS}}^{\prime}(Q)\right)^{2} \frac{F_{Q / 2, Q / 2}^{S L}\left[\begin{array}{c}
\bar{\alpha} \alpha \\
\alpha
\end{array}\right]_{e}^{e}}{\left|s_{\mathrm{NS}}(Q)\right|^{2}}(1-z)^{\Delta_{Q / 2}-2 \Delta_{\alpha} \bar{z}^{\Delta_{Q / 2}-2 \Delta_{\alpha}} \ln ^{-3 / 2}}\left(\frac{1}{(1-z)}\right) \ln ^{-3 / 2}\left(\frac{1}{\bar{z}}\right),
\end{aligned}
$$

where the exact expression for $F_{Q / 2, Q / 2}^{S L}\left[\begin{array}{c}\bar{\alpha} \alpha \\ \alpha \\ \alpha\end{array}\right]$ is revisited in Appendix A 8. We have used the late time limit to extract the leading contribution in the final step of Eq. (74). One can show that the contribution from the odd parity part of the conformal block $F_{t}^{o}\left(\Delta_{i=1,2,3,4}, \Delta_{p}, z\right)$ in the late time limit will be subleading [48] and we drop the subleading contributions in Eq. (74). 
Then the ratio associated with the second REE in super-Liouville field theory can be defined as follows:

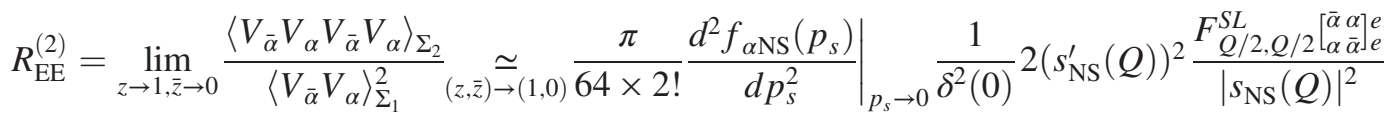

$$
\begin{aligned}
& \times(1-z)^{\Delta_{Q / 2}} \bar{z}^{\Delta_{Q / 2}} \ln ^{-3 / 2}\left(\frac{1}{(1-z)}\right) \ln ^{-3 / 2}\left(\frac{1}{\bar{z}}\right) .
\end{aligned}
$$

Then the corresponding second REE in the late time limit is

$$
\begin{aligned}
S_{\mathrm{EE}}^{(2)}\left[V_{\alpha}|0\rangle\right](t \rightarrow \infty) \underset{(z, \bar{z}) \rightarrow(1,0)}{\simeq} & -\log \left(\left.\frac{\pi}{64 \times 2 !} \frac{1}{\delta^{2}(0)} \frac{d^{2} f_{\alpha \mathrm{NS}}\left(p_{s}\right)}{d p_{s}^{2}}\right|_{p_{s} \rightarrow 0} 2\left(s_{\mathrm{NS}}^{\prime}(Q)\right)^{2} \frac{F_{Q / 2, Q / 2}^{S L}\left[\begin{array}{c}
\bar{\alpha} \alpha \\
\alpha \\
\alpha
\end{array}\right]^{e}}{\left|s_{\mathrm{NS}}(Q)\right|^{2}}\right. \\
& \left.\times(1-z)^{\Delta_{Q / 2}} \bar{z}^{\Delta_{Q / 2}} \ln ^{-3 / 2}\left(\frac{1}{(1-z)}\right) \ln ^{-3 / 2}\left(\frac{1}{\bar{z}}\right)\right) .
\end{aligned}
$$

Choosing the same corresponding reference state that we used in the early time situation, the late time of the difference $\Delta S_{\mathrm{EE}}^{(2)}\left[V_{\alpha}|0\rangle, V_{\alpha_{r}}|0\rangle\right]$ is

$$
\begin{aligned}
& \Delta S_{\mathrm{EE}}^{(2)}\left[V_{\alpha}|0\rangle, V_{\alpha_{r}}|0\rangle\right](t \rightarrow \infty)=S_{\mathrm{EE}}^{(2)}\left[V_{\alpha}|0\rangle\right](t \rightarrow \infty)-S_{\mathrm{EE}}^{(2)}\left[V_{\alpha_{r}}|0\rangle\right](t \rightarrow \infty), \\
& =-\left.\log \left(\frac{f_{\alpha \mathrm{NS}}^{\prime \prime}(p) F_{Q / 2, Q / 2}^{S L}\left[\begin{array}{c}
\bar{\alpha} \alpha \\
\alpha \bar{\alpha}
\end{array}\right]}{f_{\alpha_{r}{ }_{\mathrm{NS}}}^{\prime \prime}(p) F_{Q / 2, Q / 2}^{S L}\left[\begin{array}{c}
\bar{r}_{r} \\
\alpha_{r} \bar{\alpha}_{r}
\end{array}\right]}\right)\right|_{p \rightarrow 0}, \\
& \alpha, \alpha_{r} \in\{\alpha \mid \alpha=Q / 2+i p, p \in \mathbb{R}\} \bigcup\{\alpha \mid Q / 2>\operatorname{Re}(\alpha)>Q / 4\} \bigcup\{\alpha \mid Q / 4>\operatorname{Re}(\alpha)>0\} .
\end{aligned}
$$

Additionally, we have to consider the marginal case:

(i) $\operatorname{Re}(\alpha)=\frac{Q}{4}, \operatorname{Im}(\alpha) \neq 0$ (marginal case).

This case is similar to that mentioned above, except that $C_{\mathrm{NS}}\left(\bar{\alpha}, \alpha, \frac{Q}{2}\right)^{2}$ does not vanish at $\alpha_{s}=Q / 2$, so we have

$$
\begin{aligned}
& \left\langle V_{\bar{\alpha}}(0) V_{\alpha}(z) V_{\bar{\alpha}}(1) V_{\alpha}(\infty)\right\rangle_{\Sigma_{1}} \\
& \underset{(z, \bar{z}) \rightarrow(1,0)}{\simeq} C_{\mathrm{NS}}\left(\bar{\alpha}, \alpha, \frac{Q}{2}\right)^{2} \int_{R} F_{\alpha_{s}=Q / 2+i p_{s}, \alpha_{t}=Q / 2+i p_{t}}^{S L}\left[\begin{array}{l}
\alpha \alpha \\
\alpha \alpha
\end{array}\right]_{e}^{e}(1-z)^{\Delta_{2 \alpha}-2 \Delta_{\alpha}+p_{s}^{2}}(\bar{z})^{\Delta_{2 \alpha}-2 \Delta_{\alpha}+p_{t}^{2}} d p_{s} d p_{t} \\
& \underset{(z, \bar{z}) \rightarrow(1,0)}{\simeq} \frac{\pi}{16 \times 2} C_{\mathrm{NS}}\left(\bar{\alpha}, \alpha, \frac{Q}{2}\right)^{2} 2\left(s_{\mathrm{NS}}^{\prime}(Q)\right)^{2} \frac{F_{Q / 2, Q / 2}^{S L}\left[\begin{array}{c}
\alpha \alpha \\
\alpha \alpha
\end{array}\right] e}{]_{e}} \\
& \times(1-z)^{\Delta_{2 \alpha}-2 \Delta_{\alpha}}(\bar{z})^{\Delta_{2 \alpha}-2 \Delta_{\alpha}} \ln ^{-\frac{1}{2}}\left(\frac{1}{(1-z)}\right) \ln ^{-\frac{3}{2}}\left(\frac{1}{\bar{z}}\right) .
\end{aligned}
$$

For $\operatorname{Re}(\alpha)=\frac{Q}{4}$, in the late time limit, the ratio for the second REE reads

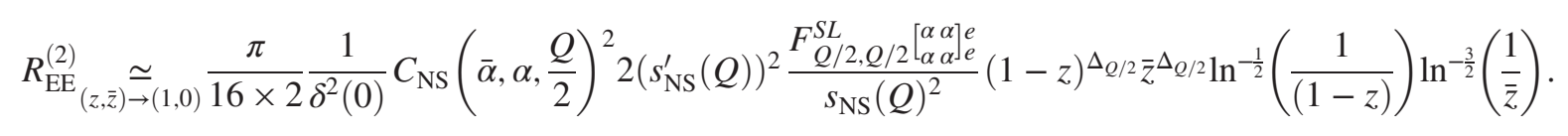

Then

$$
\begin{aligned}
& S_{\mathrm{EE}}^{(2)}\left[V_{\alpha}|0\rangle\right](t \rightarrow \infty) \underset{(z, \bar{z}) \rightarrow(1,0)}{\underset{\sim}{\simeq}}-\log \left[\frac{\pi}{16 \times 2} \frac{1}{\delta^{2}(0)} C_{\mathrm{NS}}\left(\bar{\alpha}, \alpha, \frac{Q}{2}\right)^{2} 2\left(s_{\mathrm{NS}}^{\prime}(Q)\right)^{2} \frac{\left.F_{Q / 2, Q / 2}^{S L} L_{\alpha \alpha}^{\alpha \alpha}\right]_{e}^{e}}{s_{\mathrm{NS}}(Q)^{2}}\right. \\
& \left.(1-z)^{\Delta_{Q / 2}} \bar{z}^{\Delta_{Q / 2}} \ln ^{-\frac{1}{2}}\left(\frac{1}{(1-z)}\right) \ln ^{-\frac{3}{2}}\left(\frac{1}{\bar{z}}\right)\right] \text {. }
\end{aligned}
$$

Finally,

$$
\begin{aligned}
& \Delta S_{\mathrm{EE}}^{(2)}\left[V_{\alpha}|0\rangle, V_{\alpha_{r}}|0\rangle\right](t \rightarrow \infty)=S_{\mathrm{EE}}^{(2)}\left[V_{\alpha}|0\rangle\right](t \rightarrow \infty)-S_{\mathrm{EE}}^{(2)}\left[V_{\alpha_{r}}|0\rangle\right](t \rightarrow \infty)=-\log \left(\left.\frac{f_{\alpha \mathrm{NS}}(p) F_{Q / 2, Q / 2}^{S L}\left[\begin{array}{c}
\bar{\alpha} \alpha \\
\alpha \bar{\alpha}
\end{array}\right]}{f_{\alpha_{r} \mathrm{NS}}(p) F_{Q / 2, Q / 2}^{S L}\left[\begin{array}{c}
\bar{x}_{r} \alpha_{r} \\
\alpha_{r} \bar{\alpha}_{r}
\end{array}\right)}\right|_{p \rightarrow 0}\right. \\
& \alpha, \alpha_{r} \in\{a \in \mathbb{C} \mid \operatorname{Re}(a)=Q / 4, \operatorname{Im}(a) \in \mathbb{R}, \operatorname{Im}(\alpha) \neq 0\} .
\end{aligned}
$$


One can show that we have to choose an appropriate reference state in terms of classification (23) in LFT and SLFT. Then the early time and late time behavior of $\Delta S_{\mathrm{EE}}^{(2)}\left[V_{\alpha}|0\rangle, V_{\alpha_{r}}|0\rangle\right](t)$ can be well defined and the quasiparticle picture can be restored. That also means that the divergent behavior of REE of local excited states in LFT and SLFT is quite different from that in the vacuum state, although the vacuum state is excluded in LFT and SLFT, as argued in [44]. Our calculations have classified the divergent behaviors of the entanglement entropy of local excited states, especially in LFT and SLFT.

\section{THE $n$th REE IN LFT AND SLFT}

In the previous section, we computed the second REE of the local excited states. In this subsection, we use the $n$ point conformal block and operator product expansion
(OPE) to obtain the $n$th Rényi entanglement entropy of the local excited states. Here we give a sketch of the $n$th REE following [4], which is similar to the procedure for rational CFTs, albeit with slight modification. First we define the following matrix elements $F_{\alpha_{s} \alpha_{t}}$ [similar to the $F$ matrix in Eq. (2.10) in [49] ] by

$$
F\left(\alpha_{s} \mid 1-z\right)=\int_{\alpha_{t}} F_{\alpha_{s} \alpha_{t}} \cdot F\left(\alpha_{t} \mid z\right),
$$

where $F(\alpha \mid z)$ is the conformal block for the four-point function $\left\langle V_{\bar{\alpha}}\left(z_{1}, \bar{z}_{1}\right) \cdots V_{\alpha}\left(z_{4}, \bar{z}_{4}\right)\right\rangle$. One should note that the fusion matrix is of infinite dimension which is different from that in rational CFTs.

In the late time limit, the dominant contribution from the intermediate channel in Eq. (82) is denoted by $F_{\alpha}$ and it is defined as follows:

$$
\begin{aligned}
F_{\alpha} \sim F_{\alpha_{s}=Q / 2, \alpha_{t}=Q / 2}^{L, S L}\left[\begin{array}{c}
\bar{\alpha} \alpha \\
\alpha \bar{\alpha}
\end{array}\right] \\
\quad \times \begin{cases}(1-z)^{\Delta_{Q / 2} \bar{z}^{\Delta_{Q / 2}} \ln ^{-3 / 2}\left(\frac{1}{(1-z)}\right) \ln ^{-3 / 2}\left(\frac{1}{\bar{z}}\right)} & \alpha \in\{\alpha \mid \alpha=Q / 2+i p, p \in \mathbb{R}\} \\
& \bigcup\{\alpha \mid Q / 2>\operatorname{Re}(\alpha)>Q / 4\} \bigcup\{\alpha \mid Q / 4>\operatorname{Re}(\alpha)>0\}, \\
(1-z)^{\Delta_{Q / 2} \bar{z}^{\Delta_{Q / 2}} \ln ^{-\frac{1}{2}}\left(\frac{1}{(1-z)}\right) \ln ^{-\frac{3}{2}}\left(\frac{1}{\bar{z}}\right)} & \alpha \in\{\alpha \mid \operatorname{Re}(\alpha)=Q / 4, \operatorname{Im}(\alpha) \neq 0\},\end{cases}
\end{aligned}
$$

where $\sim$ denotes neglect of the normalization factors of two-point functions and the factors associated with structure constants $C\left(\alpha_{1}, \alpha_{2}, \alpha_{3}\right)$ and $C_{\mathrm{NS}}\left(\alpha_{1}, \alpha_{2}, \alpha_{3}\right)$ in LFT and SLFT, respectively. ${ }^{13}$

The $n$th Rényi entanglement entropy can be obtained from the formula (16). We find

$$
\left\langle V_{\bar{\alpha}}\left(w_{1}, \bar{w}_{1}\right) V_{\alpha}\left(w_{2}, \bar{w}_{2}\right) \cdots V_{\alpha}\left(w_{2 n}, \bar{w}_{2 n}\right)\right\rangle_{\Sigma_{n}}=n^{-4 n \Delta} \cdot(r s)^{-2(n-1) \Delta} \cdot\left\langle V_{\bar{\alpha}}\left(z_{1}, \bar{z}_{1}\right) V_{\alpha}\left(z_{2}, \bar{z}_{2}\right) \cdots V_{\alpha}\left(z_{2 n}, \bar{z}_{2 n}\right)\right\rangle_{\Sigma_{1}},
$$

where we define

$$
\left|z_{2 k+1}\right|^{n}=r, \quad\left|z_{2 k+2}\right|^{n}=s .
$$

We normalize the two-point function ${ }^{14}$

$$
\left\langle V_{\bar{\alpha}}\left(w_{1}, \bar{w}_{1}\right) V_{\alpha}\left(w_{2}, \bar{w}_{2}\right)\right\rangle_{\Sigma_{1}}=\frac{1}{\left|w_{12}\right|^{4 \Delta}}=\frac{1}{(2 \epsilon)^{4 \Delta}} .
$$

Then we get

$$
\begin{aligned}
\frac{\left\langle V_{\bar{\alpha}}\left(w_{1}, \bar{w}_{1}\right) V_{\alpha}\left(w_{2}, \bar{w}_{2}\right) \cdots V_{\alpha}\left(w_{2 n}, \bar{w}_{2 n}\right)\right\rangle_{\Sigma_{n}}}{\left(\left\langle V_{\bar{\alpha}}\left(w_{1}, \bar{w}_{1}\right) V_{\alpha}\left(w_{2}, \bar{w}_{2}\right)\right\rangle_{\Sigma_{1}}\right)^{n}} & =\left(\frac{2 \epsilon}{n}\right)^{4 \Delta n} \cdot(r s)^{-2(n-1) \Delta} \cdot\left\langle V_{\bar{\alpha}}\left(z_{1}, \bar{z}_{1}\right) V_{\alpha}\left(z_{2}, \bar{z}_{2}\right) \cdots V_{\alpha}\left(z_{2 n}, \bar{z}_{2 n}\right)\right\rangle_{\Sigma_{1}} \\
& \rightarrow\left(\frac{2 \epsilon}{n t^{\frac{n-1}{n}}}\right)^{4 \Delta n} \cdot\left\langle V_{\alpha}\left(z_{1}, \bar{z}_{1}\right) V_{\alpha}\left(z_{2}, \bar{z}_{2}\right) \cdots V_{\alpha}\left(z_{2 n}, \bar{z}_{2 n}\right)\right\rangle_{\Sigma_{1}},
\end{aligned}
$$

where we take the late time limit in the final expression.

\footnotetext{
${ }^{13}$ Refer to Eqs. (54) and (58) in LFT and Eqs. (74) and (78) in SLFT. All these factors are divergent in the late time limit, namely, $(z, \bar{z}) \rightarrow(1,0)$.

${ }^{14}$ For the sake of simplicity, we have omitted the normalization factor associated with $\delta(0)$, which does not affect the final conclusions.
} 
The $2 n$ points $z_{1}, z_{2}, \ldots, z_{n}$ in the $z$ coordinate are given by

$$
\begin{aligned}
& z_{2 k+1}=e^{2 \pi i \frac{k}{n}}(i \epsilon+t-l)^{\frac{1}{n}}=e^{2 \pi i \frac{k+1 / 2}{n}}(l-t-i \epsilon)^{\frac{1}{n}} \\
& z_{2 k+2}=e^{2 \pi i \frac{k}{n}}(-i \epsilon+t-l)^{\frac{1}{n}}=e^{2 \pi i \frac{k+1 / 2}{n}}(l-t+i \epsilon)^{\frac{1}{n}}, \\
& \bar{z}_{2 k+1}=e^{-2 \pi i \frac{k}{n}}(-i \epsilon-t-l)^{\frac{1}{n}}=e^{-2 \pi i \frac{k+1 / 2}{n}}(l+t+i \epsilon)^{\frac{1}{n}} \\
& \bar{z}_{2 k+2}=e^{-2 \pi i \frac{k}{n}}(i \epsilon-t-l)^{\frac{1}{n}}=e^{-2 \pi i \frac{k+1 / 2}{n}}(l+t-i \epsilon)^{\frac{1}{n}} .
\end{aligned}
$$

In the early time limit $t \ll l$ we find

$$
z_{2 k+1} \rightarrow z_{2 k+2}, \quad \bar{z}_{2 k+1} \rightarrow \bar{z}_{2 k+2},
$$

for all $k$. On the other hand, if we take the late time limit $t \gg l$, we find the asymmetric limit:

$$
z_{2 k+1} \rightarrow z_{2 k+4}, \quad \bar{z}_{2 k+1} \rightarrow \bar{z}_{2 k+2},
$$

for all $k$.

If we can regard the $2 n$-point functions as $n$ products of two-point functions (86) in the late time limit, we have

$$
\begin{aligned}
& \left\langle V_{\bar{\alpha}}\left(z_{1}, \bar{z}_{1}\right) \cdots V_{\alpha}\left(z_{n}, \bar{z}_{n}\right)\right\rangle_{\Sigma_{1}} \\
& \rightarrow\left\langle V_{\bar{\alpha}}\left(z_{1}, \bar{z}_{1}\right) V_{\alpha}\left(z_{2}, \bar{z}_{2}\right)\right\rangle_{\Sigma_{1}} \otimes\left\langle V_{\bar{\alpha}}\left(z_{3}, \bar{z}_{3}\right) V_{\alpha}\left(z_{4}, \bar{z}_{4}\right)\right\rangle_{\Sigma_{1}} \\
& \quad \otimes \cdots \otimes\left\langle V_{\bar{\alpha}}\left(z_{2 n-1}, \bar{z}_{2 n-1}\right) V_{\alpha}\left(z_{2 n}, \bar{z}_{2 n}\right)\right\rangle_{\Sigma_{1}},
\end{aligned}
$$

which respects the late limit in the antiholomorphic sectors and $\otimes$ denotes that we only consider the dominant contribution with a divergent factor. In the holomorphic sector, we would like to take the late time limit. To this end, we need to exchange some of the $z_{i}$ 's with $z_{j}$ 's as

$$
\left(z_{1}, z_{2}\right)\left(z_{3}, z_{4}\right) \cdots\left(z_{2 n-1}, z_{2 n}\right) \rightarrow\left(z_{1}, z_{4}\right)\left(z_{3}, z_{6}\right) \cdots\left(z_{2 n-1}, z_{2}\right) .
$$

This transformation is realized by applying the $F$ transformation $n-1$ times.

We can estimate the difference in the late time limit as follows:

$$
\begin{aligned}
& z_{2 k+1}-z_{2 k+4} \simeq-e^{2 \pi i \frac{k+1 / 2}{n}} \cdot \frac{2 i \epsilon t^{(1 / n-1)}}{n}, \\
& \bar{z}_{2 k+1}-\bar{z}_{2 k+2} \simeq e^{-2 \pi i \frac{k+1 / 2}{n}} \cdot \frac{2 i \epsilon t^{(1 / n-1)}}{n} .
\end{aligned}
$$

Their absolute values are all the same and are given by

$$
\delta \equiv \frac{2 \epsilon}{n t^{\frac{n-1}{n}}} .
$$

Thus we can estimate the $2 n$-point function in the late time limit as follows:

$$
\begin{aligned}
& \left\langle V_{\bar{\alpha}}\left(z_{1}, \bar{z}_{1}\right) V_{\alpha}\left(z_{2}, \bar{z}_{2}\right) \cdots V_{\alpha}\left(z_{2 n}, \bar{z}_{2 n}\right)\right\rangle_{\Sigma_{1}} \\
& \quad \simeq\left(F_{\alpha}\right)^{n-1} \cdot\left[\operatorname { l i m } _ { | z _ { 2 k + 1 } - z _ { 2 k + 2 } | = \delta \rightarrow 0 } \left\langleV_{\bar{\alpha}}\left(z_{1}, \bar{z}_{1}\right) V_{\alpha}\left(z_{2}, \bar{z}_{2}\right)\right.\right. \\
& \left.\left.\quad \cdots V_{\alpha}\left(z_{2 n}, \bar{z}_{2 n}\right)\right\rangle_{\Sigma_{1}}\right] \\
& \simeq\left(F_{\alpha}\right)^{n-1} \cdot \delta^{-4 \Delta n} .
\end{aligned}
$$

Then

$$
\frac{\left\langle V_{\bar{\alpha}}\left(w_{1}, \bar{w}_{1}\right) V_{\alpha}\left(w_{2}, \bar{w}_{2}\right) \cdots V_{\alpha}\left(w_{2 n}, \bar{w}_{2 n}\right)\right\rangle_{\Sigma_{n}}}{\left(\left\langle V_{\bar{\alpha}}\left(w_{1}, \bar{w}_{1}\right) V_{\alpha}\left(w_{2}, \bar{w}_{2}\right)\right\rangle_{\Sigma_{1}}\right)^{n}}=\left(F_{\alpha}\right)^{n-1} .
$$

The late time limit of the difference of the $n$th REE $\Delta S_{A}^{(n)}\left[V_{\alpha}|0\rangle, V_{\alpha_{r}}|0\rangle\right]$ is

$$
\begin{aligned}
& \Delta S_{A}^{(n)}\left[V_{\alpha}|0\rangle, V_{\alpha_{r}}|0\rangle\right](t \rightarrow \infty)
\end{aligned}
$$

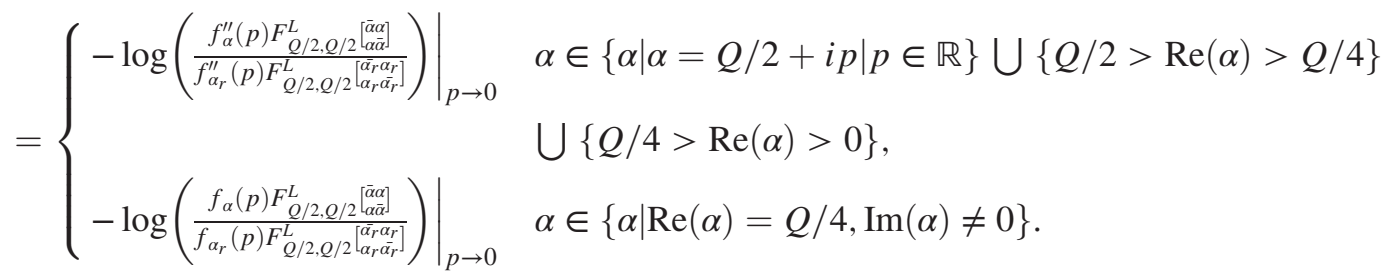

Then the variation of the difference $\Delta S_{A}^{(n)}\left[V_{\alpha}|0\rangle, V_{\alpha_{r}}|0\rangle\right]$ between the early time and late time limit is

$$
\begin{aligned}
& \Delta S_{A}^{(n)}\left[V_{\alpha}|0\rangle, V_{\alpha_{r}}|0\rangle\right](t \rightarrow \infty)-\Delta S_{A}^{(n)}\left[V_{\alpha}|0\rangle, V_{\alpha_{r}}|0\rangle\right](t \rightarrow 0)
\end{aligned}
$$

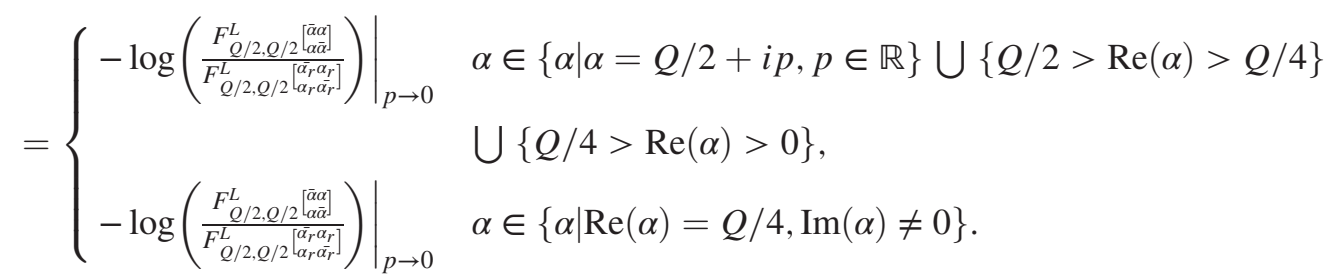

Here we have restored the structure constants in $F_{\alpha}$ defined by Eq. (98). 
This is the main difference between LFT and rational CFTs. In Appendices A 7 and A 8, unlike that in rational CFTs, one can show that Eq. (83) cannot be identified as a quantum dimension [4], which is associated with modular invariance [34]. Therefore, Eq. (98) is no longer associated with a topological quantity.

To close this section, we would like to comment on how to extend the calculation of $\Delta S_{A}^{(n)}\left[V_{\alpha}|0\rangle, V_{\alpha_{r}}|0\rangle\right](t \rightarrow \infty)-$ $\Delta S_{A}^{(n)}\left[V_{\alpha}|0\rangle, V_{\alpha_{r}}|0\rangle\right](t \rightarrow 0)$ in generic CFTs. In terms of the main result, Eq. (98), $\Delta S_{A}^{(n)}\left[V_{\alpha}|0\rangle, V_{\alpha_{r}}|0\rangle\right](t \rightarrow \infty)-$ $\Delta S_{A}^{(n)}\left[V_{\alpha}|0\rangle, V_{\alpha_{r}}|0\rangle\right](t \rightarrow 0)$ depends on the ratio of the fusion matrix elements associated with target states and reference states. Technically speaking, these fusion matrix elements come from the intermediate dominant channel in the late time limit. Then the $\Delta S_{A}^{(n)}\left[V_{\alpha}|0\rangle, V_{\alpha_{r}}|0\rangle\right](t \rightarrow \infty)-$ $\Delta S_{A}^{(n)}\left[V_{\alpha}|0\rangle, V_{\alpha_{r}}|0\rangle\right](t \rightarrow 0)$ can be obtained directly in generic CFTs. In this sense, to obtain the memory effect of REE in generic CFTs, we only care about the fusion matrix element associated with the intermediate dominant holomorphic channel in the late time limit.

\section{THE $\boldsymbol{n}$ th REE FOR GENERIC DESCENDENT STATES}

In the previous sections, we computed the $n$th REE of two classes of local excited states in LFT and SLFT, respectively. In this section, we would like to extend the analysis to states generated by generic descendent operators in LFT following [36]. In the late time limit, the authors of [36] showed that the difference of the $n$th REE only depends on the most singular term in the two-point function and the $2 n$-point function. One can define the following generic descendent operator:

$$
\tilde{V}(w, \bar{w})=L^{(-)} V_{\alpha}(w, \bar{w}),
$$

where $L^{(-)}$is a complicated product form of the holomorphic generators; $V_{\alpha}(w, \bar{w})$ is a primary operator of conformal dimension $h$; and $\tilde{V}(w, \bar{w})$ is a quasiprimary operator. The operator $L^{(-)}$has a fixed conformal dimension $m$ :

$$
\left[L_{0}, L^{(-)}\right]=m L^{(-)} .
$$

The conformal transformation for the descendant operators can be derived from the energy-momentum tensor and the conformal transformation from the $z$ plane to the $w$ plane is

$$
\left.L_{-m}\right|_{w_{1}}=\left.L_{-m}\right|_{z_{1}}\left(\frac{\partial z_{1}}{\partial w_{1}}\right)^{n}+\cdots
$$

where the ellipsis denotes the terms of lower conformal dimensions leading to less divergent terms in the limit $\epsilon \rightarrow 0$. By the conformal transformation, the four-point function is transformed as follows:

$$
\begin{aligned}
& \left\langle\tilde{V}^{+}\left(w_{1}, \bar{w}_{1}\right) \tilde{V}\left(w_{1}^{\prime}, \bar{w}_{1}^{\prime}\right) \tilde{V}^{+}\left(w_{2}, \bar{w}_{2}\right) \tilde{V}\left(w_{2}^{\prime}, \bar{w}_{2}^{\prime}\right)\right\rangle \\
& \quad=\left(\frac{\partial w_{1}}{\partial z_{1}}\right)^{h+m}\left(\frac{\partial \bar{w}_{1}}{\partial \bar{z}_{1}}\right)^{h+\bar{m}}\left(\frac{\partial w_{2}}{\partial z_{2}}\right)^{h+m}\left(\frac{\partial \bar{w}_{2}}{\partial \bar{z}_{2}}\right)^{h+\bar{m}}\left(\frac{\partial w_{1}^{\prime}}{\partial z_{1}^{\prime}}\right)^{h+m}\left(\frac{\partial \bar{w}_{1}^{\prime}}{\partial \bar{z}_{1}^{\prime}}\right)^{h+\bar{m}}\left(\frac{\partial w_{2}^{\prime}}{\partial z_{2}^{\prime}}\right)^{h+m}\left(\frac{\partial \bar{w}_{2}^{\prime}}{\partial \bar{z}_{2}^{\prime}}\right)^{h+\bar{m}} \\
& \left\langle\tilde{V}^{+}\left(z_{1}, \bar{z}_{1}\right) \tilde{V}\left(z_{1}^{\prime}, \bar{z}_{1}^{\prime}\right) \tilde{V}^{+}\left(z_{2}, \bar{z}_{2}\right) \tilde{V}\left(z_{2}^{\prime}, \bar{z}_{2}^{\prime}\right)\right\rangle+\text { less divergent terms. }
\end{aligned}
$$

The coefficient for the leading term is the same as that for the primary operator. The terms with lower conformal dimensions do not change the final result.

The two-point function for $V$ can be expressed as follows:

$$
\left\langle\left(L^{(-)} V_{\alpha}\right)^{+}(z), L^{(-)} V_{\alpha}\left(z^{\prime}\right)\right\rangle=\frac{(-1)^{m}\left\langle h\left|L^{(-) \dagger} L^{(-)}\right| h\right\rangle}{\left(z-z^{\prime}\right)^{2(h+m)}} .
$$

In the late time limit, $\left(z_{1}, z_{2}^{\prime}\right)\left(z_{2}, z_{1}^{\prime}\right)\left(\bar{z}_{1}, \bar{z}_{1}^{\prime}\right)\left(\bar{z}_{2}, \bar{z}_{2}^{\prime}\right)$ approach each other. The four-point correlation function of $V$ can be transformed to

$$
\begin{aligned}
& \left\langle\tilde{V}^{+}\left(z_{1}, \bar{z}_{1}\right) \tilde{V}\left(z_{1}^{\prime}, \bar{z}_{1}^{\prime}\right) \tilde{V}^{+}\left(z_{2}, \bar{z}_{2}\right) \tilde{V}\left(z_{2}^{\prime}, \bar{z}_{2}^{\prime}\right)\right\rangle \\
& \quad=\mathcal{D}\left\langle V_{\bar{\alpha}}\left(z_{1}, \bar{z}_{1}\right) V_{\alpha}\left(z_{1}^{\prime}, \bar{z}_{1}^{\prime}\right) V_{\bar{\alpha}}\left(z_{2}, \bar{z}_{2}\right) V_{\alpha}\left(z_{2}^{\prime}, \bar{z}_{2}^{\prime}\right)\right\rangle \\
& \quad=\mathcal{D} \int_{m}^{\prime} c_{m}\left\langle\left. V_{\bar{\alpha}}\left(z_{1}\right) V_{\alpha}\left(z_{1}^{\prime}\right)\right|_{m} V_{\bar{\alpha}}\left(z_{2}\right) V_{\alpha}\left(z_{2}^{\prime}\right)\right\rangle\left\langle\left. V_{\bar{\alpha}}\left(\bar{z}_{1}\right) V_{\alpha}\left(\bar{z}_{1}^{\prime}\right)\right|_{m} V_{\bar{\alpha}}\left(\bar{z}_{2}\right) V_{\alpha}\left(\bar{z}_{2}^{\prime}\right)\right\rangle \\
& \quad=\mathcal{D} \int_{m, n}^{\prime} c_{m, n}\left\langle\left. V_{\bar{\alpha}}\left(z_{1}\right) V_{\alpha}\left(z_{2}^{\prime}\right)\right|_{m} V_{\bar{\alpha}}\left(z_{2}\right) V_{\alpha}\left(z_{1}^{\prime}\right)\right\rangle\left\langle\left. V_{\bar{\alpha}}\left(\bar{z}_{1}\right) V_{\alpha}\left(\bar{z}_{1}^{\prime}\right)\right|_{n} V_{\bar{\alpha}}\left(\bar{z}_{2}\right) V_{\alpha}\left(\bar{z}_{2}^{\prime}\right)\right\rangle \\
& \quad=\int_{m, n}^{\prime} c_{m, n}\left\langle\left. L^{(-)} V_{\alpha}^{+}\left(z_{1}\right) L^{(-)} V_{\alpha}\left(z_{2}^{\prime}\right)\right|_{m} L^{(-)} V_{\alpha}^{+}\left(z_{2}\right) L^{(-)} V_{\alpha}\left(z_{1}^{\prime}\right)\right\rangle\left\langle\left. V_{\bar{\alpha}}\left(\bar{z}_{1}\right) V_{\alpha}\left(\bar{z}_{1}^{\prime}\right)\right|_{n} V_{\bar{\alpha}}\left(\bar{z}_{2}\right) V_{\alpha}\left(\bar{z}_{2}^{\prime}\right)\right\rangle .
\end{aligned}
$$


Here $\left\langle\left. V_{\bar{\alpha}}\left(z_{1}\right) V_{\alpha}\left(z_{1}^{\prime}\right)\right|_{m} V_{\bar{\alpha}}\left(z_{2}\right) V_{\alpha}\left(z_{2}^{\prime}\right)\right\rangle$ denotes the conformal block expansion with the Virasoro module $[m]$, which exhibits a continuous spectrum in Liouville field theory, and $[m]$ contains the dominant contribution satisfying the fusion rule. ${ }^{15}$ The correlation function of four descendant operators has been transformed into the differential operator $\mathcal{D}$ acting on the correlation function of corresponding primaries in the first equality. In the second equality, the partition function is expanded by the conformal blocks and here $c_{m}$ denotes the OPE coefficients. In the third equality, the holomorphic part can be expressed by the $t$-channel like in [4] in the late time limit. In the fourth equality, we pull the differential operator back into the Virasoro operators acting on the primaries in the correlation function. In LFT and SLFT, we have shown that the dominant contribution to REE in the late time limit comes from the intermediate channel with $m=Q / 2, n=Q / 2$ in primed contour integration over $m, n$ in Eq. (104).

In Secs. II and III, we have already found that the most divergent term comes from the intermediate operator with the minimal conformal dimension of the operator in the intermediate fusion channel and $c_{\operatorname{Min}\left(\alpha_{s}\right), \operatorname{Min}\left(\alpha_{t}\right)}=$ $\left.\left.\left(F_{\operatorname{Min}\left(\alpha_{s}\right), \operatorname{Min}\left(\alpha_{t}\right)}^{L}\right]_{\alpha \bar{\alpha}}^{\bar{\alpha} \alpha}\right]\right)^{-1}=\frac{1}{F_{\alpha}^{L, S L},}{ }^{16}$ which is similar to that in rational CFTs [4]. We use upper index $L, S L$ to distinguish the quantities in LFT and SLFT. The most divergent term is as follows:

$$
\begin{aligned}
\left\langle\tilde{V}^{+}\left(z_{1}, \bar{z}_{1}\right) \tilde{V}\left(z_{1}^{\prime}, \bar{z}_{1}^{\prime}\right) \tilde{V}^{+}\left(z_{2}, \bar{z}_{2}\right) \tilde{V}\left(z_{2}^{\prime}, \bar{z}_{2}^{\prime}\right)\right\rangle= & \frac{1}{\left.\left.F_{\operatorname{Min}\left(\alpha_{s}\right), \operatorname{Min}\left(\alpha_{t}\right)}^{L, S L \alpha}\right)_{\alpha}^{\bar{\alpha}}\right]}\left\langle L^{(-)} V_{\alpha}^{+}\left(z_{1}\right) L^{(-)} V_{\alpha}\left(z_{2}^{\prime}\right)\right\rangle \otimes\left\langle L^{(-)} V_{\alpha}^{+}\left(z_{2}\right) L^{(-)} V_{\alpha}\left(z_{1}^{\prime}\right)\right\rangle \\
& \otimes\left\langle V_{\bar{\alpha}}\left(\bar{z}_{1}\right) V_{\alpha}\left(\bar{z}_{1}^{\prime}\right)\right\rangle \otimes\left\langle V_{\bar{\alpha}}\left(\bar{z}_{2}\right) V_{\alpha}\left(\bar{z}_{2}^{\prime}\right)\right\rangle+\text { less divergent terms. }
\end{aligned}
$$

So the four-point function in the $w$-coordinate keeping the most divergent term is

$$
\begin{aligned}
& \left\langle\tilde{V}^{+}\left(w_{1}, \bar{w}_{1}\right) \tilde{V}\left(w_{1}^{\prime}, \bar{w}_{1}^{\prime}\right) \tilde{V}^{+}\left(w_{2}, \bar{w}_{2}\right) \tilde{V}\left(w_{2}^{\prime}, \bar{w}_{2}^{\prime}\right)\right\rangle=\frac{1}{\left.F_{\operatorname{Min}\left(\alpha_{s}\right), \operatorname{Min}\left(\alpha_{t}\right)}^{L, S L}\right)_{\alpha \bar{\alpha}}^{\bar{\alpha} \alpha}}\left\langle L^{(-)} V_{\alpha}^{+}\left(w_{1}\right) L^{(-)} V_{\alpha}\left(w_{2}^{\prime}\right)\right\rangle \otimes\left\langle L^{(-)} V_{\alpha}^{+}\left(w_{2}\right) L^{(-)} V_{\alpha}\left(w_{1}^{\prime}\right)\right\rangle \\
& \otimes\left\langle V_{\bar{\alpha}}\left(\bar{w}_{1}\right) V_{\alpha}\left(\bar{w}_{1}^{\prime}\right)\right\rangle \otimes\left\langle V_{\bar{\alpha}}\left(\bar{w}_{2}\right) V_{\alpha}\left(\bar{w}_{2}^{\prime}\right)\right\rangle+\text { less divergent terms. }
\end{aligned}
$$

Therefore, for a quasiprimary operator, we still have $S^{(2)}[\tilde{V}|0\rangle](t \rightarrow \infty)=-\log F_{\operatorname{Min}\left(\alpha_{s}\right) \cdot \operatorname{Min}\left(\alpha_{t}\right)}^{L, S L}\left[\begin{array}{l}\bar{\alpha} \alpha \\ \alpha \bar{\alpha}\end{array}\right],{ }^{17}$ which is defined by Eq. (83) in LFT and SLFT.

Finally, we present the main results with the more generic descendent operators:

$$
\tilde{V}=\sum_{m, j, r, k} d_{m, j, r, k} \partial^{m} L^{(-, j)} \bar{\partial}^{r} \bar{L}^{(-, k)} V_{\alpha}(z, \bar{z}) .
$$

Here $j$ and $k$ denote the quasiprimary operators and $V_{\alpha}$ is the primary operator. $L^{(-, j)} \bar{L}^{(-, k)} O_{a}(w, \bar{w})$ is a quasiprimary operator and $L^{(-, j)}\left(\bar{L}^{(-, k)}\right)$ is a combination of holomorphic (or antiholomorphic) Virasoro generators with fixed conformal dimension $\left[L_{0}, L^{(-, j)}\right]=p_{j} L^{(-, j)}$.

We do not repeat the calculation in detail here as this has been analyzed in [36]. The differences have already been presented in Eq. (104). Here we provide the final result as follows:

$$
\begin{aligned}
& S_{n}[\tilde{V}|0\rangle](t \rightarrow \infty) \\
& \quad=\log F_{\operatorname{Min}\left(\alpha_{s}\right), \operatorname{Min}\left(\alpha_{t}\right)}^{L}\left[\begin{array}{c}
\bar{\alpha} \alpha \\
\alpha \bar{\alpha}
\end{array}\right]-\frac{1}{n-1} \log \operatorname{Tr} \rho_{0}^{n} \\
& \quad=S_{n}^{\text {primary }}-\frac{1}{n-1} \log \operatorname{Tr} \rho_{0}^{n},
\end{aligned}
$$

\footnotetext{
${ }^{15}$ Due to the fusion rule in LFT or SLFT, $[m]$ is not a vacuum module, which does not belong to the spectrum of LFT or SLFT.
}

with the normalized density matrix $\rho_{0}=\frac{\rho}{\operatorname{Tr} \rho}$, where

$S_{n}^{\text {primary }}\left[V_{\alpha}|0\rangle\right](t \rightarrow \infty)=-\log F_{\operatorname{Min}\left(\alpha_{s}\right), \operatorname{Min}\left(\alpha_{t}\right)}^{L}\left[\begin{array}{c}\bar{\alpha} \alpha \\ \alpha \bar{\alpha}\end{array}\right]$

is the REE of the local excited state $V_{\alpha}|0\rangle$. The density matrix is defined as

$$
\rho=B M B^{\dagger} M^{\dagger},
$$

and these matrices are associated with coefficients in Eq. (107):

$$
\begin{aligned}
B_{\{m, j\},\{r, k\}} & =d_{m, j, r, k}^{*}, \\
M_{\{m, j\},\{r, k\}} & =\left\langle h\left|L^{(-, j) \dagger} L^{(-, j)}\right| h\right\rangle \delta_{j, k} k^{r-m} .
\end{aligned}
$$

As we see from Eq. (108), $S_{n}\left[V_{\alpha}|0\rangle\right](t \rightarrow \infty)$ has a similar structure in LFT and SLFT with that in rational CFTs. $S_{n}\left[V_{\alpha}|0\rangle\right](t \rightarrow \infty)$ makes two main contributions. The first contains the universal part depending on the fusion

\footnotetext{
${ }^{16}$ Here the $F_{\alpha}^{L, S L}$ contains the divergent piece shown in Eq. (83), and $\operatorname{Min}\left(\alpha_{s}\right), \operatorname{Min}\left(\alpha_{t}\right)$ are the minimal conformal dimensions of the intermediate operator involved in the fusion process.

${ }^{17}$ Precisely, here we have neglected the normalization factors and associated DOZZ factors.
} 
matrix element of the corresponding primary operator and it is divergent due to the divergent component [Eq. (83)] in the first term. The other comes from the normalization scheme of the local descendent operator. Finally, we can see that the variation of the difference $\Delta S_{\mathrm{EE}}^{(n)}\left[V_{\alpha}|0\rangle, V_{\alpha_{r}}|0\rangle\right]$ between the early time and the late time is

$$
\begin{aligned}
& \Delta S_{\mathrm{EE}}^{(n)}\left[V_{\alpha}|0\rangle, V_{\alpha_{r}}|0\rangle\right](t \rightarrow \infty)-\Delta S_{\mathrm{EE}}^{(n)}\left[V_{\alpha}|0\rangle, V_{\alpha_{r}}|0\rangle\right](t \rightarrow 0)
\end{aligned}
$$

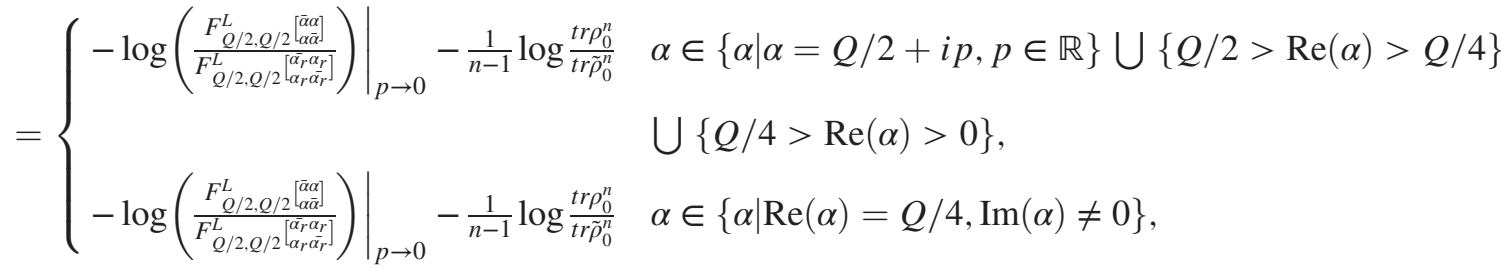

where we choose $\tilde{\rho}_{0}$ defined by the reference state (107) associated with primary operator $V_{\alpha_{r}}$.

\section{DISCUSSIONS AND CONCLUSIONS}

In this paper, the time evolution of the difference $\Delta S_{\mathrm{EE}}^{(n)}\left[V_{\alpha}|0\rangle, V_{\alpha_{r}}|0\rangle\right](t)$ of REE between locally excited states $V_{\alpha}|0\rangle$ and the reference states $V_{\alpha_{r}}|0\rangle$ has been studied in $1+1$-dimensional irrational CFT, especially in Liouville field theory and super-Liouville field theory. In rational CFTs, there are finite primary operators and one needs to undertake the finite summation to extract the difference of REE shown in [4]. Furthermore, the reference states $V_{\alpha_{r}}|0\rangle$ can be chosen as a vacuum state $\mathbf{1}|0\rangle$. In irrational CFTs, there are infinite-dimensional and continuous spectra which are also highly degenerate. One might doubt that the differences of REE in irrational CFTs have very different structures compared with that [4] in rational CFTs.

To answer this question, we calculate the second REE in a compact $c=1$ boson at a generic radius which is an irrational CFT as a preliminary exercise. Because the Hilbert spaces of LFT and SLFT are very special in irrational CFTs, they are especially chosen as playgrounds to calculate the difference of REE $\Delta S_{\mathrm{EE}}^{(n)}\left[V_{\alpha}|0\rangle, V_{\alpha_{r}}|0\rangle\right](t)$ between two excited states, e.g., $V_{\alpha_{r}}|0\rangle$ and $V_{\alpha_{r}}|0\rangle$. Furthermore, there is no well-studied holographic dual of the local primary operator in integrable rational CFTs [50] and one cannot make use of AdS/CFT to calculate REE in this case. Although the holographic dual of LFT or SLFT is not clear, it is interesting to extract the large $c$ universal properties thereof. One can study the time evolution of REE $S_{\mathrm{EE}}^{(n)}\left[V_{\alpha}|0\rangle\right]$ by applying the replica trick in LFT and SLFT and one might obtain some properties of large $c$ CFTs.

To understand these properties, the second REE $S_{\mathrm{EE}}^{(2)}\left[V_{\alpha}|0\rangle\right]$ of local excited states is calculated in LFT and SLFT. For a state excited by a local primary operator $V_{\alpha}$, the REE is divergent both in the early and late time limits. The divergent behaviors of REE $S_{\mathrm{EE}}^{(2)}\left[V_{\alpha}|0\rangle\right](t)$ in the early and late time limits are different, which seems to contradict the quasiparticle picture proposed in rational CFTs $[4,7]$. That also means that $S_{\mathrm{EE}}^{(2)}\left[V_{\alpha}|0\rangle\right](t \rightarrow \infty)-S_{\mathrm{EE}}^{(2)}\left[V_{\alpha}|0\rangle\right](t \rightarrow 0)$ is divergent. ${ }^{18}$ The identity operator does not live in the Hilbert space of LFT and SLFT and no discrete terms contribute to the REE; the vacuum block does not make a contribution to REE. That is the main reason leading to the different divergent behavior of REE in the early and late time limits. To define finite quantities, e.g., $\Delta S_{A}^{(n)}\left[V_{\alpha}|0\rangle, V_{\alpha_{r}}|0\rangle\right](t)$, one has to classify all locally excited states in LFT and SLFT. The zero point of the structure constant (DOZZ formula) presented in the second REE has been estimated to classify the primary operators in LFT and SLFT. These primary operators have been divided into two classes in terms of the real part of Liouville momentum $\alpha$, e.g., $\alpha \in\{\alpha \mid \alpha=Q / 2+i p, p \in$ $\mathbb{R}\} \bigcup\{\alpha \mid Q / 2>\operatorname{Re}(\alpha)>Q / 4\} \bigcup\{\alpha \mid Q / 4>\operatorname{Re}(\alpha)>0\}$ and $\alpha \in\{\alpha \mid \operatorname{Re}(\alpha)=Q / 4, \operatorname{Im}(\alpha) \neq 0\}$. Due to the fact that the second REE of excited states is divergent, one has to choose an appropriate reference state $V_{\alpha_{r}}|0\rangle$ which lives in the same class as the target states $V_{\alpha}|0\rangle$. The difference $\Delta S_{A}^{(2)}\left[V_{\alpha}|0\rangle, V_{\alpha_{r}}|0\rangle\right](t)$ of the second REE between target states $V_{\alpha}|0\rangle$ and reference states $V_{\alpha_{r}}|0\rangle$ will be finite in both the early and late limits. One can study the time evolution behavior of $\Delta S_{A}^{(2)}\left[V_{\alpha}|0\rangle, V_{\alpha_{r}}|0\rangle\right](t)$. The difference of the REE $\Delta S_{A}^{(2)}\left[V_{\alpha}|0\rangle, V_{\alpha_{r}}|0\rangle\right]$ between the early time limit and the late time limit always coincides with the log of the ratio

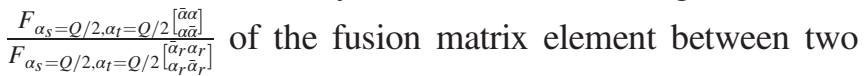
excited states, e.g., $V_{\alpha}|0\rangle, V_{\alpha_{r}}|0\rangle$. The precise expression has been listed in Eq. (112). Following [4], one can also directly extend this analysis to the generic $n$th REE. The difference $\Delta S_{A}^{(n)}\left[V_{\alpha}|0\rangle, V_{\alpha_{r}}|0\rangle\right](t)$ between the early and late time is independent of $n$ and it still contains the log of the ratio of

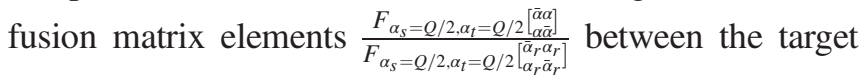
and reference state. Finally, REE of the generic descendent states following [36] has been investigated. Comparing this with the case of primary states, the difference

\footnotetext{
${ }^{18}$ Equivalently, $\Delta S_{A}^{(n)}\left[V_{\alpha}|0\rangle, 1|0\rangle\right](t \rightarrow \infty)-\Delta S_{A}^{(n)}\left[V_{\alpha}|0\rangle, 1|0\rangle\right] \times$ $(t \rightarrow 0)$ is divergent.
} 
$\Delta S_{A}^{(n)}\left[\tilde{V}_{\alpha}|0\rangle, \tilde{V}_{\alpha_{r}}|0\rangle\right](t)$ in descendent states $\tilde{V}_{\alpha}|0\rangle, \tilde{V}_{\alpha_{r}}|0\rangle$ in LFT or SLFT now contains one more additional term which is associated with the normalization factor of the descendent operator.

How can one understand the different divergence behaviors of $\Delta S_{A}^{(n)}\left[\tilde{V}_{\alpha}|0\rangle, \tilde{V}_{\alpha_{r}}|0\rangle\right]$ in the two classes of local excited states? In terms of [43], they define the states $V_{\alpha}|0\rangle$ as the Liouville momentum $\alpha \in\{Q / 2+i p, p \in \mathbb{R}\}$ as normalizable and the states $V_{\alpha}|0\rangle$ with $0<\alpha<Q / 2$ are nonnormalizable states. The normalizable states correspond to nonlocal operators which create macroscopic holes in the surface. In this paper, we have confirmed the difference between the normalizable states and non-normalizable states from the memory effect of the REE perspective.

Finally, one can apply these techniques to calculate the out-of-time-ordered correlation function (OTOC) to check whether the superintegrability of LFT is consistent with the chaotic proposal [51]. Recently, the authors of [52] proposed a correspondence between $1+1$-dimensional Liouville field theory and the one-dimensional conformal quantum mechanics Sachdev-Ye-Kitaev (SYK) model. One can compare the OTOC in Liouville field theory with the late time behavior of the two-point function of bilocal operators in SYK and then check the correspondence [52] between LFT and SYK. More recently, the authors of [53-55] proposed the Liouville field theory action as the optimization of the complexity of static states in conformal field theory. Also, the associated measurements of the complexity in generic field theory and the holographic aspects thereof have been proposed in [56-62], respectively. One can extend the study of the complexity of local excited states in conformal field theory to define the optimization procedure. Hopefully, some progress in these directions can be reported in the near future.

\section{ACKNOWLEDGMENTS}

We would like to thank Konstantin Aleshkin, Vladimir Belavin, X. Cao, Bin Chen, Harald Dorn, M. R. Gaberdiel,
Wu-Zhong Guo, George Jorjadze, Li Li, Zhu-Xi Luo, Tokiro Numasawa, Hao-Yu Sun, J. Teschner, Kento Watanabe, and Jie-qiang $\mathrm{Wu}$ for their discussions and suggestions during various stages of the project. We appreciate Harald Dorn, Xing Huang, Axel Kleinschmidt, Hermann Nicolai, and Tadashi Takayanagi, who commented on the draft. We especially thank Xing Huang and Stefan Theisen for intensive discussions during the whole project. S. H. appreciates Axel Kleinschmidt, Hermann Nicolai, Stefan Theisen, and Tadashi Takayanagi for their encouragement and support. S. H. is supported by the Max-Planck fellowship in Germany, the German-Israeli Foundation for Scientific Research and Development, and the National Natural Science Foundation of China (Grant No. 11305235).

\section{APPENDIX: NOTATIONS AND TECHNIQUES}

\section{The Notations of LFT}

The full Liouville action (see a review in [46]) is

$$
S_{L}=\frac{1}{4 \pi} \int d^{2} \xi \sqrt{g}\left[\partial_{a} \phi \partial_{b} \phi g^{a b}+Q R \phi+4 \pi \mu e^{2 b \phi}\right],
$$

where $Q=b+\frac{1}{b}$. The conformal dimension of the corresponding primary operator $e^{2 \alpha \phi}$ is

$$
\Delta\left(e^{2 \alpha \phi}\right)=\bar{\Delta}\left(e^{2 \alpha \phi}\right)=\alpha(Q-\alpha) .
$$

The stress tensor is

$$
T(z)=-(\partial \phi)^{2}+Q \partial^{2} \phi,
$$

and the central charge of the conformal algebra is

$$
c_{L}=1+6 Q^{2}=1+6\left(b+b^{-1}\right)^{2} .
$$

The three-point function of the primary operator in LFT is

$$
\left\langle V_{\alpha_{1}}\left(z_{1}, \bar{z}_{1}\right) V_{\alpha_{2}}\left(z_{2}, \bar{z}_{2}\right) V_{\alpha_{3}}\left(z_{3}, \bar{z}_{3}\right)\right\rangle=\frac{C\left(\alpha_{1}, \alpha_{2}, \alpha_{3}\right)}{\left|z_{12}\right|^{2\left(\Delta_{1}+\Delta_{2}-\Delta_{3}\right)}\left|z_{13}\right|^{2\left(\Delta_{1}+\Delta_{3}-\Delta_{2}\right)}\left|z_{23}\right|^{2\left(\Delta_{2}+\Delta_{3}-\Delta_{1}\right)}},
$$

with $z_{i j}=z_{i}-z_{j}$. The function $C\left(\alpha_{1}, \alpha_{2}, \alpha_{3}\right)$ is called structure constants associated with dynamical data of any CFT. The DOZZ formula is an analytic expression for $C$ in LFT from $[63,64]$. The DOZZ formula gives the three-point function

$$
C\left(\alpha_{1}, \alpha_{2}, \alpha_{3}\right)=\lambda^{\left(Q-\sum_{i=1}^{3} \alpha_{i}\right) / b} \frac{\Upsilon_{b}^{\prime}(0) \Upsilon_{b}\left(2 \alpha_{1}\right) \Upsilon_{b}\left(2 \alpha_{2}\right) \Upsilon_{b}\left(2 \alpha_{3}\right)}{\Upsilon_{b}\left(\alpha_{1}+\alpha_{2}+\alpha_{3}-Q\right) \Upsilon_{b}\left(\alpha_{1}+\alpha_{2}-\alpha_{3}\right) \Upsilon_{b}\left(\alpha_{2}+\alpha_{3}-\alpha_{1}\right) \Upsilon_{b}\left(\alpha_{3}+\alpha_{1}-\alpha_{2}\right)},
$$

where

$$
\Upsilon_{b}(x)=\frac{1}{\Gamma_{b}(x) \Gamma_{b}(Q-x)}
$$

and

$$
\lambda=\pi \mu \gamma\left(b^{2}\right) b^{2-2 b^{2}}
$$


The $\Gamma_{b}(x)$ is given by Eq. (A31). In this paper, we need the four-point function of the primary operator, which reads

$$
\left\langle V_{\alpha_{1}}\left(z_{1}, \bar{z}_{1}\right) V_{\alpha_{2}}\left(z_{2}, \bar{z}_{2}\right) V_{\alpha_{3}}\left(z_{3}, \bar{z}_{3}\right) V_{\alpha_{4}}\left(z_{4}, \bar{z}_{4}\right)\right\rangle=\left|z_{13}\right|^{2\left(\Delta_{4}-\Delta_{1}-\Delta_{2}-\Delta_{3}\right)}\left|z_{14}\right|^{2\left(\Delta_{2}+\Delta_{3}-\Delta_{1}-\Delta_{4}\right)}\left|z_{24}\right|^{-4 \Delta_{2}}\left|z_{34}\right|^{2\left(\Delta_{1}+\Delta_{2}-\Delta_{3}-\Delta_{4}\right)} G_{1234}(z, \bar{z}),
$$

with the harmonic cross ratio $z$ defined as

$$
z=\frac{z_{12} z_{34}}{z_{13} z_{24}}
$$

and $G_{1234}(z, \bar{z})$ as

$$
G_{1234}(z, \bar{z})=\frac{1}{2} \int_{-\infty}^{\infty} \frac{d p}{2 \pi} C\left(\alpha_{1}, \alpha_{2}, Q / 2+i p\right) R_{L}\left(\alpha_{s}\right) C\left(\alpha_{3}, \alpha_{4}, Q / 2-i p\right) F_{1234}\left(\Delta_{i}, \Delta_{p}, z\right) F_{1234}\left(\Delta_{i}, \Delta_{p}, \bar{z}\right) .
$$

Here $\Delta_{p}=p^{2}+Q^{2} / 4$, and the functions $F_{1234}(z)$ and $F_{1234}(\bar{z})$ are the Virasoro conformal block. In this paper, we follow the notation for the four-point Green function given in [45] with respect to the normalization of the twopoint function.

\section{The Notations of SLFT}

The $\mathcal{N}=1$ supersymmetric Liouville field theory may be defined by the action (see a review in [46])

$$
\begin{aligned}
S_{S L}= & \frac{1}{4 \pi} \int d^{2} \xi \sqrt{g} \frac{1}{2 \pi} g_{a b} \partial_{a} \varphi \partial_{b} \varphi \\
& +\frac{1}{2 \pi}(\psi \bar{\partial} \psi+\bar{\psi} \partial \bar{\psi})+2 i \mu b^{2} \bar{\psi} \psi e^{b \varphi}+2 \pi \mu^{2} b^{2} e^{2 b \varphi},
\end{aligned}
$$

where $\varphi$ is a bosonic and $\psi$ a fermionic field, $\mu$ denotes a two-dimensional cosmological constant, and $b$ is a Liouville coupling constant.

The theory has $\mathcal{N}=1$ superconformal symmetry. The energy-momentum tensor and the superconformal current are

$$
\begin{aligned}
T & =-\frac{1}{2}\left(\partial \varphi \partial \varphi-Q \partial^{2} \varphi+\psi \partial \psi\right), \\
G & =i(\psi \partial \varphi-Q \partial \psi),
\end{aligned}
$$

and the superconformal algebra is

$$
\begin{aligned}
& {\left[L_{m}, L_{n}\right]=(m-n) L_{m+n}+\frac{c}{12} m\left(m^{2}-1\right) \delta_{m+n},} \\
& {\left[L_{m}, G_{k}\right]=\frac{m-2 k}{2} G_{m+k},} \\
& \left\{G_{k}, G_{l}\right\}=2 L_{l+k}+\frac{c}{3}\left(k^{2}-\frac{1}{4}\right) \delta_{k+l} .
\end{aligned}
$$

The central charge in SLFT is given by

$$
c=\frac{3}{2}+3 Q^{2}
$$

The NS-NS primary fields $e^{\alpha \varphi(z, \bar{z})}$ in the $\mathcal{N}=1$ SLFT have conformal dimensions

$$
\Delta_{\alpha}^{\mathrm{NS}}=\frac{1}{2} \alpha(Q-\alpha)
$$

As before, physical states have $\alpha=\frac{Q}{2}+i p$ with $Q=b+\frac{1}{b}$. The R-R primary field is defined as

$$
R_{\alpha}^{\epsilon}(z, \bar{z})=\sigma^{\epsilon}(z, \bar{z}) e^{\alpha \varphi(z, \bar{z})},
$$

where $\sigma$ is the spin field and $\epsilon= \pm$ is the fermion parity. For simplicity we can take all $\epsilon=+$ and drop this index.

The dimension of the R-R operator is

$$
\Delta_{\alpha}^{R}=\frac{1}{16}+\frac{1}{2} \alpha(Q-\alpha)
$$

To consider both NS and R sectors, we will need various functions defined differently for each sector. Here we use the notations $C_{i}, \Upsilon_{i}, \Gamma_{i}$, where $i=1 \mathrm{mod}$ 2 for $C_{\mathrm{NS}}, \Upsilon_{\mathrm{NS}}, \Gamma_{\mathrm{NS}}$ and $i=0 \bmod 2$ for $\mathrm{R}$. One can refer to Appendix A 4 to find out the exact definition of these special functions.

The four-point function for the NS-NS operator is $G_{4}(z, \bar{z})=\left\langle V_{\alpha_{4}}(\infty, \infty) V_{\alpha_{3}}(1,1) V_{\alpha_{2}}(z, \bar{z}) V_{\alpha_{1}}(0,0)\right\rangle$,

which can be written in the "s-channel" representation:

$$
\begin{aligned}
& G_{4}(z, \bar{z})= \int_{\frac{Q}{2}+i \mathbb{R}^{+}} \frac{d \alpha_{s}}{i}\left[C\left(\alpha_{4}, \alpha_{3}, \alpha_{s}\right)\right. \\
& \times C\left(\bar{\alpha}_{s}, \alpha_{2}, \alpha_{1}\right)\left|F_{\alpha_{s}}^{\mathrm{e}}\left[\begin{array}{c}
\alpha_{3} \alpha_{2} \\
\alpha_{4} \alpha_{1}
\end{array}\right](z)\right|^{2} \\
&\left.-\tilde{C}\left(\alpha_{4}, \alpha_{3}, \alpha_{s}\right) \tilde{C}\left(\bar{\alpha}_{s}, \alpha_{2}, \alpha_{1}\right)\left|F_{\alpha_{s}}^{\mathrm{o}}\left[\begin{array}{l}
\alpha_{3} \alpha_{2} \\
\alpha_{4} \alpha_{1}
\end{array}\right](z)\right|^{2}\right] .
\end{aligned}
$$


The e,o in $F_{\alpha_{s}}^{\mathrm{e}}$ and $F_{\alpha_{s}}^{\mathrm{o}}$ denote $\mathcal{N}=1$ Neveu-Schwarz blocks with even and odd fermion parity as in $[65,66]$.

Following $[67,68]$ the structure constants have the following explicit form (here, $\alpha$ stands for $\alpha_{1}+\alpha_{2}+\alpha_{3}$ ):

$$
\begin{aligned}
& C\left(\alpha_{1}, \alpha_{2}, \alpha_{3}\right)=\left(\pi \mu \gamma\left(\frac{Q b}{2}\right) b^{1-b^{2}}\right)^{(Q-\alpha) / b} \frac{\Upsilon_{\mathrm{NS}}^{\prime}(0) \Upsilon_{\mathrm{NS}}\left(2 \alpha_{1}\right) \Upsilon_{\mathrm{NS}}\left(2 \alpha_{2}\right) \Upsilon_{\mathrm{NS}}\left(2 \alpha_{3}\right)}{\Upsilon_{\mathrm{NS}}(\alpha-Q) \Upsilon_{\mathrm{NS}}\left(\alpha_{1+2-3}\right) \Upsilon_{\mathrm{NS}}\left(\alpha_{2+3-1}\right) \Upsilon_{\mathrm{NS}}\left(\alpha_{3+1-2}\right)} \\
& \tilde{C}\left(\alpha_{1}, \alpha_{2}, \alpha_{3}\right)=\left(\pi \mu \gamma\left(\frac{Q b}{2}\right) b^{1-b^{2}}\right)^{(Q-\alpha) / b} \frac{2 i \Upsilon_{\mathrm{NS}}^{\prime}(0) \Upsilon_{\mathrm{NS}}\left(2 \alpha_{1}\right) \Upsilon_{\mathrm{NS}}\left(2 \alpha_{2}\right) \Upsilon_{\mathrm{NS}}\left(2 \alpha_{3}\right)}{\Upsilon_{\mathrm{R}}(\alpha-Q) \Upsilon_{\mathrm{R}}\left(\alpha_{1+2-3}\right) \Upsilon_{\mathrm{R}}\left(\alpha_{2+3-1}\right) \Upsilon_{\mathrm{R}}\left(\alpha_{3+1-2}\right)},
\end{aligned}
$$

where we define $\alpha_{i+j-k}=\alpha_{i}+\alpha_{j}-\alpha_{k}$ for short and

$$
\begin{aligned}
& \Upsilon_{1}(x) \equiv \Upsilon_{\mathrm{NS}}(x)=\Upsilon_{b}\left(\frac{x}{2}\right) \Upsilon_{b}\left(\frac{x+Q}{2}\right)=\frac{1}{\Gamma_{\mathrm{NS}}(x) \Gamma_{\mathrm{NS}}(Q-x)}, \\
& \Gamma_{1}(x) \equiv \Gamma_{\mathrm{NS}}(x)=\Gamma_{b}\left(\frac{x}{2}\right) \Gamma_{b}\left(\frac{x+Q}{2}\right) .
\end{aligned}
$$

Functions for the R sector are defined differently. For example, we have

$$
\Gamma_{0}(x) \equiv \Gamma_{\mathrm{R}}(x)=\Gamma_{b}\left(\frac{x+b}{2}\right) \Gamma_{b}\left(\frac{x+b^{-1}}{2}\right) .
$$

The four-point function for R-R operators,

$$
G_{4}(z, \bar{z})=\left\langle R_{\alpha_{4}}(\infty, \infty) R_{\alpha_{3}}(1,1) R_{\alpha_{2}}(z, \bar{z}) R_{\alpha_{1}}(0,0)\right\rangle
$$

can be also written in a similar form:

$$
G_{4}(z, \bar{z})=\int_{\frac{\rho}{2}+i \mathbb{R}^{+}} \frac{d \alpha_{s}}{i}\left[C_{R}^{+}\left(\alpha_{4}, \alpha_{3} \mid \alpha_{s}\right) C_{R}^{+}\left(\alpha_{2}, \alpha_{1} \mid \bar{\alpha}_{s}\right)\left|F_{\alpha_{s}}^{\mathrm{e}}\left[{ }_{\alpha_{4}}^{\alpha_{3} \alpha_{2}}\right](z)\right|^{2}-\tilde{C}_{R}^{+}\left(\alpha_{4}, \alpha_{3} \mid \alpha_{s}\right) \tilde{C}_{R}^{+}\left(\alpha_{2}, \alpha_{1} \mid \bar{\alpha}_{s}\right) \mid F_{\alpha_{s}}^{\mathrm{o}}\left[{ }_{\alpha_{4}}^{\alpha_{3} \alpha_{1}}\right]\left[\left.(z)\right|^{2}\right] .\right.
$$

The corresponding structure constants become

$$
\begin{aligned}
C_{R}^{\epsilon}\left(\alpha_{1}, \alpha_{2} \mid \alpha_{3}\right)= & \left(\pi \mu \gamma\left(\frac{Q b}{2}\right) b^{1-b^{2}}\right)^{(Q-\alpha) / b} \times\left[\frac{\Upsilon_{\mathrm{NS}}^{\prime}(0) \Upsilon_{R}\left(2 \alpha_{1}\right) \Upsilon_{R}\left(2 \alpha_{2}\right) \Upsilon_{\mathrm{NS}}\left(2 \alpha_{3}\right)}{\Upsilon_{R}(\alpha-Q) \Upsilon_{R}\left(\alpha_{1+2-3}\right) \Upsilon_{\mathrm{NS}}\left(\alpha_{2+3-1}\right) \Upsilon_{\mathrm{NS}}\left(\alpha_{3+1-2}\right)}\right. \\
& \left.+\frac{\epsilon \Upsilon_{\mathrm{NS}}^{\prime}(0) \Upsilon_{R}\left(2 \alpha_{1}\right) \Upsilon_{R}\left(2 \alpha_{2}\right) \Upsilon_{\mathrm{NS}}\left(2 \alpha_{3}\right)}{\Upsilon_{\mathrm{NS}}(\alpha-Q) \Upsilon_{\mathrm{NS}}\left(\alpha_{1+2-3}\right) \Upsilon_{R}\left(\alpha_{2+3-1}\right) \Upsilon_{R}\left(\alpha_{3+1-2}\right)}\right] \\
\tilde{C}_{R}^{\epsilon}\left(\alpha_{1}, \alpha_{2} \mid \alpha_{3}\right)= & -\frac{i \epsilon}{2}\left[\left(p_{1}^{2}+p_{2}^{2}\right) C_{R}^{\epsilon}\left(\alpha_{1}, \alpha_{2} \mid \alpha_{3}\right)-2 p_{1} p_{2} C_{R}^{-\epsilon}\left(\alpha_{1}, \alpha_{2} \mid \alpha_{3}\right)\right] .
\end{aligned}
$$

\section{The function $\Gamma_{b}(x)$}

The function $\Gamma_{b}(x)$ is a close relative of the double gamma function studied in [69]. It can be expressed by means of the integral representation

$$
\log \Gamma_{b}(x)=\int_{0}^{\infty} \frac{d t}{t}\left(\frac{e^{-x t}-e^{-Q t / 2}}{\left(1-e^{-b t}\right)\left(1-e^{-t / b}\right)}-\frac{(Q-2 x)^{2}}{8 e^{t}}-\frac{Q-2 x}{t}\right) .
$$

Important properties of $\Gamma_{b}(x)$ are listed as follows:

$$
\text { functional relation } \Gamma_{b}(x+b)=\sqrt{2 \pi} b^{b x-\frac{1}{2}} \Gamma^{-1}(b x) \Gamma_{b}(x) .
$$

analyticity $\Gamma_{b}(x)$ is a meromorphic function and it has poles only 


$$
\text { at } x=-n b-m b^{-1}, n, m \in \mathbb{Z}^{\geq 0} \text {. }
$$

Further properties are listed in [70].

In terms of the functional relation in Eq. (A32), one can find the residues near by various pole as follows:

$$
\Gamma_{b}(x)=\frac{\Gamma_{b}(Q)}{2 \pi x}+O(x), \quad x \rightarrow 0 .
$$

\section{Double Sine Function}

In terms of $\Gamma_{b}(x)$, the double sine function is given as follows:

$$
S_{b}(x)=\frac{\Gamma_{b}(x)}{\Gamma_{b}(Q-x)}
$$

We will use the properties

$$
\text { Self-duality } S_{b}(x)=S_{b^{-1}}(x)
$$

Functional relation $S_{b}\left(x+b^{ \pm 1}\right)=2 \sin \left(\pi b^{ \pm 1} x\right) S_{b}(x)$,

Reflection relation $S_{b}(x) S_{b}(Q-x)=1$.

The asymptotics behavior of $S_{b}(x)$ is

$$
S_{b}\left(x=x_{0}+\mathrm{i} x_{1}\right) \sim \begin{cases}e^{\pi x_{1}\left(\frac{b}{2}+\frac{1}{2 b}-x_{0}\right)} e^{i \pi\left(\frac{b^{2}}{12}+\frac{1}{12 b^{2}}-\frac{b x_{0}}{2}-\frac{x_{0}}{2 b}+\frac{x_{0}^{2}}{2}-\frac{x_{1}^{2}}{2}+\frac{1}{4}\right)} & \text { for }|x| \rightarrow \infty, x_{1}<0, \\ e^{-\pi x_{1}\left(\frac{b}{2}+\frac{1}{2 b}-x_{0}\right)} e^{-i \pi\left(\frac{b^{2}}{12}+\frac{1}{12 b^{2}}-\frac{b x_{0}}{2}-\frac{x_{0}}{2 b}+\frac{x_{0}^{2}}{2}-\frac{x_{1}^{2}}{2}+\frac{1}{4}\right)} & \text { for }|x| \rightarrow \infty, x_{1}>0 .\end{cases}
$$

We define the following functions:

$$
\Upsilon_{b}(x)=\frac{1}{\Gamma_{b}(x) \Gamma_{b}(Q-x)}, \quad S_{b}(x)=\frac{\Gamma_{b}(x)}{\Gamma_{b}(Q-x)}, \quad G_{b}(x)=\mathrm{e}^{-\frac{i \pi}{2} x(Q-x)} S_{b}(x),
$$

and, in SFLT, we follow notations from [71] to denote

$$
\begin{aligned}
\Gamma_{\mathrm{NS}}(x)=\Gamma_{b}\left(\frac{x}{2}\right) \Gamma_{b}\left(\frac{x+Q}{2}\right), & \Gamma_{\mathrm{R}}(x)=\Gamma_{b}\left(\frac{x+b}{2}\right) \Gamma_{b}\left(\frac{x+b^{-1}}{2}\right), \\
\Upsilon_{\mathrm{NS}}(x)=\Upsilon_{b}\left(\frac{x}{2}\right) \Upsilon_{b}\left(\frac{x+Q}{2}\right), & \Upsilon_{\mathrm{R}}(x)=\Upsilon_{b}\left(\frac{x+b}{2}\right) \Upsilon_{b}\left(\frac{x+b^{-1}}{2}\right),
\end{aligned}
$$

etc. Using relations, basic properties of these functions can be established easily:

$$
\begin{gathered}
\frac{\Gamma_{\mathrm{NS}}(2 \alpha)}{\Gamma_{\mathrm{NS}}(2 \alpha-Q)}=W_{\mathrm{NS}}(\alpha) \lambda^{\frac{Q-2 \alpha}{2 b}}, \\
\frac{\Gamma_{\mathrm{R}}(2 \alpha)}{\Gamma_{\mathrm{R}}(2 \alpha-Q)}=W_{\mathrm{R}}(\alpha) \lambda^{\frac{Q-2 \alpha}{2 b}},
\end{gathered}
$$

where $W_{\mathrm{NS}}(\alpha), W_{\mathrm{R}}(\alpha)$ are defined in (A45) and (A46), and $\lambda=\pi \mu \gamma\left(\frac{b Q}{2}\right) b^{1-b^{2}}$. The functions $W_{i}$ are defined as

$W_{\mathrm{NS}}(\alpha)=\frac{2(\pi \mu \gamma(b Q / 2))^{-\frac{Q-2 \alpha}{2 b}} \pi(\alpha-Q / 2)}{\Gamma(1+b(\alpha-Q / 2)) \Gamma\left(1+\frac{1}{b}(\alpha-Q / 2)\right)}$,

$W_{R}(\alpha)=\frac{2 \pi(\pi \mu \gamma(b Q / 2))^{\frac{Q-2 \alpha}{2 b}}}{\Gamma(1 / 2+b(\alpha-Q / 2)) \Gamma\left(1 / 2+\frac{1}{b}(\alpha-Q / 2)\right)}$.
In the literature, one can define the following equations for convenience:

$$
\begin{gathered}
S_{1}(x) \equiv S_{\mathrm{NS}}(x)=\frac{\Gamma_{\mathrm{NS}}(x)}{\Gamma_{\mathrm{NS}}(Q-x)}, \\
S_{0}(x) \equiv S_{\mathrm{R}}(x)=\frac{\Gamma_{\mathrm{R}}(x)}{\Gamma_{\mathrm{R}}(Q-x)} .
\end{gathered}
$$

They have the following relations with $S_{\mathrm{NS}, R}$ functions:

$$
\begin{gathered}
\frac{S_{\mathrm{NS}}(2 x)}{S_{\mathrm{NS}}(2 x-Q)}=W_{\mathrm{NS}}(x) W_{\mathrm{NS}}(Q-x), \\
\frac{S_{\mathrm{R}}(2 x)}{S_{\mathrm{R}}(2 x-Q)}=W_{R}(x) W_{R}(Q-x) .
\end{gathered}
$$

In the paper we used the following:

(i) Reflection properties:

$$
S_{\mathrm{NS}}(x) S_{\mathrm{NS}}(Q-x)=S_{\mathrm{R}}(x) S_{\mathrm{R}}(Q-x)=1
$$


(ii) Locations of zeros and poles can be obtained from Eq. (A41):

$$
\begin{gathered}
S_{\mathrm{NS}}(x)=0 \Leftrightarrow x=Q+m b+n b^{-1}, \quad m, n \in \mathbb{Z}_{\geq 0}, m+n \in 2 \mathbb{Z}, \\
S_{\mathrm{R}}(x)=0 \Leftrightarrow x=Q+m b+n b^{-1}, \quad m, n \in \mathbb{Z}_{\geq 0}, m+n \in 2 \mathbb{Z}+1, \\
S_{\mathrm{NS}}(x)^{-1}=0 \Leftrightarrow x=-m b-n b^{-1}, \quad m, n \in \mathbb{Z}_{\geq 0}, m+n \in 2 \mathbb{Z}, \\
S_{\mathrm{R}}(x)^{-1}=0 \Leftrightarrow x=-m b-n b^{-1}, \quad m, n \in \mathbb{Z}_{\geq 0}, m+n \in 2 \mathbb{Z}+1 .
\end{gathered}
$$

(iii) Basic residue:

$$
\lim _{x \rightarrow 0} x S_{\mathrm{NS}}(x)=\frac{1}{\pi}
$$

\section{Poles Structure and Discrete Terms}

Following the Appendix about the LFT in [72], the four-point functions of primary operators are

$$
\left\langle V_{\alpha_{1}}(0) V_{\alpha_{2}}(z) V_{\alpha_{3}}(1) V_{\alpha_{4}}(\infty)\right\rangle=\int_{i \mathbb{R}+\frac{Q}{2}} C\left(\alpha_{1}, \alpha_{2}, \alpha_{s}\right) C\left(\alpha_{3}, \alpha_{4}, \bar{\alpha}_{s}\right)\left|F_{s}\left(\Delta_{\alpha_{i=1,2,3,4}}, \Delta_{\alpha_{s}}, z\right)\right|^{2} d \alpha_{s},
$$

where $\operatorname{Re}\left(\alpha_{1}\right), \ldots, \operatorname{Re}\left(\alpha_{4}\right)=Q / 2$. For $\alpha_{i} \in(0, Q / 2)$ cases, Eq. (A57) needs to be extended. The proper way to integrate should preserve the crossing symmetry with the assumptions given in [73-75].

The integrand of (A57), as a function of $\alpha \in \mathbb{C}$, has many poles. The two structure constants $C\left(\alpha_{1}, \alpha_{2}, \alpha_{s}\right)$ and $C\left(\alpha_{3}, \alpha_{4}, \bar{\alpha}_{s}\right)$ in (A57) have poles and these poles comes from the zeros of the $\Upsilon$ 's in the denominator of the DOZZ formula in LFT. ${ }^{19}$ These poles are as follows:

\begin{tabular}{c|c|c|c|c|c|c|c}
\hline 1 & 2 & 3 & 4 & 5 & 6 & 7 & 8 \\
\hline$Q-\alpha_{s}-L$ & $\alpha_{s}+L$ & $2 Q-\alpha_{s}-L$ & $-Q+\alpha_{s}+L$ & $\alpha_{d}-L$ & $-\alpha_{d}+Q+L$ & $-\alpha_{d}-L$ & $\alpha_{d}+Q+L$ \\
\hline
\end{tabular}

where $\alpha_{s} \equiv \alpha_{1}+\alpha_{2}, \alpha_{d} \equiv \alpha_{1}-\alpha_{2}$, for LFT: $L=\left\{b m+b^{-1} n: m, n \in \mathbb{Z}^{\geq 0}\right\}$,

for SLFT NS sector: $L=\left\{b m+b^{-1} n: m+n \in 2 \mathbb{Z}^{\geq 0}\right\}$,

for SLFT R sector: $L=\left\{b m+b^{-1} n: m+n \in 2 \mathbb{Z}^{\geq 0}+1\right\}$.

Note that rows 1 and 2 are related by $\alpha \mapsto Q-\alpha$ symmetry and so are 3 and 4 , etc. The poles coming from $C\left(\alpha_{2}, \alpha_{3}, \bar{\alpha}\right)$ are obtained by replacing $1,2 \rightarrow 3,4$ in the above equations.

If $\operatorname{Re}\left(\alpha_{i}\right)=\frac{Q}{2}$, the real part of the poles belongs to the intervals $(-\infty, 0] \cup[Q,+\infty)$ and the intervals do not intersect with the integration contour $Q / 2+i R$ (A57). When $\operatorname{Re}\left(\alpha_{s}\right)=\operatorname{Re}\left(\alpha_{1}+\alpha_{2}\right)$ starts to decrease from $Q$ into the interval $(0, Q)$, the poles start to move on the plane $\alpha_{s}$. One can show that only the rows 1 and 2 may cross the line $Q / 2+i R$. When $\operatorname{Re}\left(\alpha_{s}\right)$ decreases to $Q / 2$, row 1 crosses the line from its left, and row 2 cross the line from it right. $\operatorname{As} \operatorname{Re}\left(\alpha_{s}\right)$ further decreases, several poles from those rows in the table will have crossed the line. These poles are

$$
\begin{aligned}
& P_{+} \equiv\left\{x \in Q-\left(\alpha_{1}+\alpha_{2}\right)-L: \operatorname{Re}(x) \in(Q / 2, Q)\right\} \\
& P_{-} \equiv\left\{x \in \alpha_{1}+\alpha_{2}+L: \operatorname{Re}(x) \in(0, Q / 2)\right\}
\end{aligned}
$$

\footnotetext{
${ }^{19}$ The similar structure constant in SLFT is given in the above Appendix A 2 and we just list the relevant results.
}

To extend analytically the integral (A57), the integration contour has to be deformed to avoid the poles from crossing it. Using the Cauchy formula, this amounts to adding $\pm 2 \pi i$ times the residues of the integrand of (A57) at points in $P_{ \pm}$, respectively. These terms are the so-called discrete terms [73-75] known in the literature.

Using the $\alpha_{s} \mapsto Q-\alpha_{s}$ symmetry of $\Upsilon$, the contribution of $P_{+}$equals that of $P_{-}$. Then, the poles from $\alpha_{2,3}$ can be similarly treated. For the LFT four-point function for values of $\operatorname{Re}\left(\alpha_{i}\right) \in(0, Q / 2)$, the resulting form is as follows [72]:

$$
\begin{aligned}
& \left\langle V_{\alpha_{1}}(0) V_{\alpha_{2}}(z) V_{\alpha_{3}}(1) V_{\alpha_{4}}(\infty)\right\rangle \\
& =\int_{\frac{\underline{Q}}{2}+i \mathbb{R}} C\left(\alpha_{1}, \alpha_{2}, \alpha_{s}\right) C\left(\alpha_{3}, \alpha_{4}, \bar{\alpha}_{s}\right)\left|F_{s}\left(\Delta_{\alpha_{i}}, \Delta_{\alpha_{s}}, z\right)\right|^{2} d \alpha_{s} \\
& \quad+-2 \sum_{p \in P_{-}} \operatorname{Res}_{\alpha_{s} \rightarrow p}\left[C\left(\alpha_{1}, \alpha_{2}, \alpha_{s}\right) C\left(\alpha_{3}, \alpha_{4}, \bar{\alpha}_{s}\right)\right] \mid \\
& \quad \times\left. F_{s}\left(\Delta_{\alpha_{i}}, \Delta_{\alpha_{s}}, z\right)\right|^{2}+[(1,2) \leftrightarrow(3,4)] .
\end{aligned}
$$


In this paper, we will use asymptotic form of the conformal block as $z \rightarrow 0$, or $z \rightarrow 1$. The $z \rightarrow 0$ series expansion of the conformal block is

$$
\begin{aligned}
F_{s}\left(\Delta_{\alpha_{i}}, \Delta_{\alpha_{s}}, z\right) & =z^{-\Delta_{\alpha_{1}}-\Delta_{\alpha_{2}}+\Delta_{\alpha_{s}}} \\
& \times\left(1+\frac{\left(\Delta_{\alpha_{2}}-\Delta_{\alpha_{1}}+\Delta_{\alpha_{s}}\right)\left(\Delta_{\alpha_{3}}-\Delta_{\alpha_{4}}+\Delta_{\alpha_{s}}\right)}{2 \Delta_{\alpha_{s}}} z\right. \\
& \left.+O\left(z^{2}\right)\right) .
\end{aligned}
$$

Once $s$-channel blocks are known, $t$-channel blocks can be obtained by a permutation of the arguments, while taking global conformal symmetry into account, and this becomes [76]

$F_{t}\left(\Delta_{\alpha_{i}}, \Delta_{\alpha_{t}}, z\right)=(1-z)^{\Delta_{2}+\Delta_{3}-\Delta_{1}-\Delta_{4}} F_{s}\left(\Delta_{\alpha_{i}}, \Delta_{\alpha_{s}}, 1-z\right)$.

In this paper, to compute the dominant asymptotic behavior of (A59) as $z \rightarrow 0$ or $z \rightarrow 1$ is very important. We need to consider the internal charges $\alpha_{s} \in P_{+} \cup(Q / 2+i \mathbb{R})$ involved and find the smallest scaling dimension $\Delta_{\alpha}=\alpha(Q-\alpha)$. In this paper, we will make use of these details to obtain REE. Some further calculations have been given in Appendix A 6.

\section{To Calculate the Dominant Contribution in Early Time}

In this section, we would like to show some details about how to do the early time integral appearing in the four-point function in LFT. The early time limit $(z, \bar{z}) \rightarrow(0,0)$ is a short distance limit, or equivalently $z_{1} \rightarrow z_{2}$ or $z_{3} \rightarrow z_{4}$. Hence one can insert a corresponding OPE [74,75] in Eq. (A57). If the OPE would be given by a sum as in rational CFTs, the dominant contribution trivially would be realized by the contribution of the r.h.s. operator with the lowest conformal dimension. In LFT, we have an integral as given in formula (1.10) of [74].

For $\operatorname{Re}\left(\alpha_{1}+\alpha_{2}\right)>Q / 2$ [74], the integral is

$$
\left\langle V_{\alpha_{1}}(0) V_{\alpha_{2}}(z) V_{\alpha_{1}}(1) V_{\alpha_{2}}(\infty)\right\rangle \underset{(z, \bar{z}) \rightarrow(0,0)}{\simeq} \int_{0}^{\infty}(z \bar{z})^{P^{2}} f(P) d P
$$

whose asymptotics for $z \rightarrow 0$ is

$$
\sqrt{\frac{\pi}{-\log z}} \frac{f(0)}{2}+\frac{1}{2 \log z} f^{\prime}(0)+\frac{\sqrt{\pi}}{8(-\log z)^{\frac{3}{2}}} f^{\prime \prime}(0)+\cdots
$$

One can apply the following OPE [74]:

$$
\begin{aligned}
V_{\alpha_{1}}(0) V_{\alpha_{2}}(z)= & \int^{\prime} \frac{d P}{4 \pi} C\left(\alpha_{1}, \alpha_{2}, \alpha_{s}\right)(z \bar{z})^{\Delta_{Q / 2+i P}-\Delta_{\alpha_{1}}-\Delta_{\alpha_{2}}} \\
& \times\left[V_{Q / 2+i P}(0)\right] .
\end{aligned}
$$

The integration contour here is the real axis if $\alpha_{1}$ and $\alpha_{2}$ are in the basic domain $\left|Q / 2-\operatorname{Re}\left(\alpha_{1}\right)\right|+$ $\left|Q / 2-\operatorname{Re}\left(\alpha_{2}\right)\right|<Q / 2$. In this case, one can find that

$\frac{1}{4 \pi} \sqrt{\frac{\pi}{-\log (z \bar{z})}}(z \bar{z})^{\frac{Q^{2}}{4}-\Delta_{1}-\Delta_{2}} C\left(\alpha_{1}, \alpha_{2}, \frac{Q}{2}\right) V_{Q / 2}(0)+\cdots$

But we have to take into account that $C\left(\alpha_{1}, \alpha_{2}, \frac{Q}{2}\right)=0$. The function $f(P)$ is $C\left(\alpha_{1}, \alpha_{2}, \frac{Q}{2}+i P\right) C\left(\alpha_{1}, \alpha_{2}, \frac{Q}{2}-i P\right)$. Due to $f(0)=0$, the first nonvanishing term is the one with $f^{\prime \prime}(0)$, which is then

$$
\begin{aligned}
& \left\langle V_{\alpha_{1}}(0) V_{\alpha_{2}}(z) V_{\alpha_{1}}(1) V_{\alpha_{2}}(\infty)\right\rangle \\
& \underset{(z, \bar{z}) \rightarrow(0,0)}{\simeq} \frac{\sqrt{\pi}}{8} f^{\prime \prime}(0)(z \bar{z})^{\frac{Q^{2}}{4}-\Delta_{1}-\Delta_{2}}(-\log (z \bar{z}))^{-\frac{3}{2}} .
\end{aligned}
$$

This conclusion corresponds to a statement by Seiberg in [43]; see the comment after his Eq. (4.15). ${ }^{20}$

For $\alpha_{1}$ and $\alpha_{2}$ that stay outside of the basic domain [74], we can use the following OPE:

$$
\begin{aligned}
V_{\alpha_{1}}(0) V_{\alpha_{2}}(z)= & \frac{1}{2}(z \bar{z})^{-2 \alpha_{1} \alpha_{2}}\left[V_{\alpha_{1}+\alpha_{2}}(0)\right] \\
& +\frac{1}{2}(z \bar{z})^{-2 \alpha_{1} \alpha_{2}} S\left(\alpha_{1}+\alpha_{2}\right)\left[V_{Q-\alpha_{1}-\alpha_{2}}(0)\right] \\
& +\frac{1}{2} \int \frac{d P}{4 \pi} C\left(\alpha_{1}, \alpha_{2}, \alpha_{S}\right)(z \bar{z})^{\Delta_{Q / 2+i P}-\Delta_{\alpha_{1}}-\Delta_{\alpha_{2}}} \\
& \times\left[V_{Q / 2+i P}(0)\right] .
\end{aligned}
$$

The integral has again an asymptotics as above Eq. (A64). The first two terms in Eq. (A68) are the socalled discrete terms. But now the more dominant term is given by the contribution of the discrete term, i.e.,

$$
\begin{aligned}
& \left\langle V_{\alpha_{1}}(0) V_{\alpha_{2}}(z) V_{\alpha_{1}}(1) V_{\alpha_{2}}(\infty)\right\rangle \\
& \underset{(z, \bar{z}) \rightarrow(0,0)}{\simeq} C\left(\alpha_{1}, \alpha_{2}, \alpha_{1}+\alpha_{2}\right) C\left(\alpha_{1}, \alpha_{2}, Q-\alpha_{1}-\alpha_{2}\right) \\
& \quad(z \bar{z})^{\Delta_{\alpha_{1}+\Delta_{\alpha_{2}}}-\Delta_{1}-\Delta_{2}} V_{\alpha_{1}+\alpha_{2}}(0)
\end{aligned}
$$

Depending on the value of $\alpha_{1}$ and $\alpha_{2}$, other discrete terms can take over, as discussed in [74].

To calculate the late time limit of $\left\langle V_{\alpha_{1}}(0) V_{\alpha_{2}}(z) \times\right.$ $\left.V_{\alpha_{1}}(1) V_{\alpha_{2}}(\infty)\right\rangle$, we have to use conformal boostrap equations firstly and following the above procedures in this

\footnotetext{
${ }^{20}$ The fact that the author writes $Q^{2} / 8$ instead of $Q^{2} / 4$ is due to different normalizations.
} 
subsection. We have presented the main results and will not repeat all the details here.

\section{The Fusion Matrix in Liouville Field Theory}

In this subsection, we will see how to associate with the quantum dimension defined in LFT. For the late time limit of the second REE, the fusion matrix element $F_{Q / 2, Q / 2}^{L}\left[\begin{array}{c}\bar{\alpha} \alpha \\ \alpha \\ \alpha\end{array}\right]$ will be presented. This matrix element cannot be identified as the quantum dimension in LFT. Following the definition of the quantum dimension [40] LFT, we will show that the $F_{0,0}^{L}\left[\begin{array}{c}\bar{\alpha} \alpha \\ \alpha \bar{\alpha}\end{array}\right]$ will be the quantum dimension.

Just to follow the convention in [77-79], we introduce $F_{\alpha_{s} \alpha_{t}}^{\mathrm{L}}\left[_{\alpha_{4} \alpha_{1}}^{\alpha_{3} \alpha_{2}}\right]$ as follows:

$$
F_{\alpha_{s} \alpha_{t}}^{\mathrm{L}}\left[\begin{array}{c}
\alpha_{3} \alpha_{2} \\
\alpha_{4} \alpha_{1}
\end{array}\right]=\frac{N\left(\alpha_{s}, \alpha_{2}, \alpha_{1}\right) N\left(\alpha_{4}, \alpha_{3}, \alpha_{s}\right)}{N\left(\alpha_{t}, \alpha_{3}, \alpha_{2}\right) N\left(\alpha_{4}, \alpha_{t}, \alpha_{1}\right)} F_{\alpha_{s} \alpha_{t}}^{\mathrm{PT}}\left[\begin{array}{c}
\alpha_{3} \alpha_{2} \\
\alpha_{4} \alpha_{1}
\end{array}\right]
$$

where

$$
N\left(\alpha_{3}, \alpha_{2}, \alpha_{1}\right)=\frac{\Gamma_{b}\left(2 Q-2 \alpha_{3}\right) \Gamma_{b}\left(2 \alpha_{2}\right) \Gamma_{b}\left(2 \alpha_{1}\right) \Gamma_{b}(Q)}{\Gamma_{b}\left(2 Q-\alpha_{1}-\alpha_{2}-\alpha_{3}\right) \Gamma_{b}\left(Q-\alpha_{1}-\alpha_{2}+\alpha_{3}\right) \Gamma_{b}\left(\alpha_{1}+\alpha_{3}-\alpha_{2}\right) \Gamma_{b}\left(\alpha_{2}+\alpha_{3}-\alpha_{1}\right)} .
$$

The $b-6 j$ symbol has the explicit form

$$
\begin{aligned}
F_{\alpha_{s} \alpha_{t}}^{\mathrm{PT}}\left[\begin{array}{l}
\alpha_{3} \alpha_{2} \\
\bar{\alpha}_{4} \alpha_{1}
\end{array}\right]= & \frac{S_{b}\left(\alpha_{2}+\alpha_{s}-\alpha_{1}\right) S_{b}\left(\alpha_{t}+\alpha_{1}-\alpha_{4}\right)}{S_{b}\left(\alpha_{2}+\alpha_{t}-\alpha_{3}\right) S_{b}\left(\alpha_{s}+\alpha_{3}-\alpha_{4}\right)}\left|S_{b}\left(2 \alpha_{t}\right)\right|^{2} \int_{\mathcal{C}} d u S_{b}\left(-\alpha_{2} \pm\left(\alpha_{1}-Q / 2\right)+u\right) S_{b}\left(-\alpha_{4} \pm\left(\alpha_{3}-Q / 2\right)+u\right) \\
& \times S_{b}\left(\alpha_{2}+\alpha_{4} \pm\left(\alpha_{t}-Q / 2\right)-u\right) S_{b}\left(Q \pm\left(\alpha_{s}-Q / 2\right)-u\right)
\end{aligned}
$$

where the following notation has been used: $S_{b}(\alpha \pm u):=S_{b}(\alpha+u) S_{b}(\alpha-u)$. The function $S_{b}(x)$ is defined by Eq. (A35) in Appendix A 4. The integral can be performed using the identity

$$
\int_{i \mathbb{R}} d z \prod_{i=1}^{3} S_{b}\left(\mu_{i}-z\right) S_{b}\left(\nu_{i}+z\right)=\prod_{i, j=1}^{3} S_{b}\left(\mu_{i}+\nu_{j}\right),
$$

where the balancing condition is $\sum_{i=1}^{3} \mu_{i}+\nu_{i}=Q$. We are interested in the $F_{\alpha_{s}}^{\mathrm{PT}} \alpha_{t}\left[\begin{array}{l}\bar{\alpha} \alpha \\ \alpha\end{array}\right]$ which has been shown in Sec. II E. In terms of the notation of [80], the $6 j$ symbols correspond to $F_{\alpha_{s} \alpha_{t}}^{\mathrm{PT}}\left[\begin{array}{c}\alpha_{3} \alpha_{2} \\ \alpha_{\alpha_{1}}\end{array}\right]=\left\{\begin{array}{l}\alpha_{1} \alpha_{2} \\ \alpha_{3} \alpha_{4}\end{array} \mid \begin{array}{c}\alpha_{s} \\ \alpha_{t}\end{array}\right\}_{b}^{\text {an }}$ and the canonical $6 j$ symbols are defined as

$$
\left\{\begin{array}{l|l}
\alpha_{1} \alpha_{2} & \alpha_{s} \\
\alpha_{3} \alpha_{4} & \alpha_{t}
\end{array}\right\}_{b}=\frac{M\left(\alpha_{s}, \alpha_{2}, \alpha_{1}\right) M\left(\alpha_{4}, \alpha_{3}, \alpha_{s}\right)}{M\left(\alpha_{t}, \alpha_{3}, \alpha_{2}\right) M\left(\alpha_{4}, \alpha_{t}, \alpha_{1}\right)}\left\{\begin{array}{c|c}
\alpha_{1} \alpha_{2} & \alpha_{s} \\
\alpha_{3} \alpha_{4} & \alpha_{t}
\end{array}\right\}_{b}^{\text {an }}
$$

with

$$
M\left(\alpha_{3}, \alpha_{2}, \alpha_{1}\right)=\left(S_{b}\left(2 Q-\alpha_{1}-\alpha_{2}-\alpha_{3}\right) S_{b}\left(Q-\alpha_{1}-\alpha_{2}+\alpha_{3}\right) S_{b}\left(\alpha_{1}+\alpha_{3}-\alpha_{2}\right) S_{b}\left(\alpha_{2}+\alpha_{3}-\alpha_{1}\right)\right)^{-\frac{1}{2}} .
$$

With following relation given in [80],

$$
\left\{\begin{array}{ll}
\alpha_{1} \alpha_{2} \alpha_{s} \\
\alpha_{3} \alpha_{4} \alpha_{t}
\end{array}\right\}_{b}=\left\{\begin{array}{ll|l}
\alpha_{1} \alpha_{2} & \alpha_{s} \\
\alpha_{3} \bar{\alpha}_{4} & \alpha_{t}
\end{array}\right\}_{b}, \quad \bar{\alpha}_{4}:=Q-\alpha_{4}
$$

one can obtain that

$$
F_{\alpha_{s} \alpha_{t}}^{\mathrm{PT}}\left[\begin{array}{c}
\alpha_{3} \alpha_{2} \\
\alpha_{4} \alpha_{1}
\end{array}\right]=\frac{M\left(\alpha_{4}, \alpha_{t}, \alpha_{1}\right) M\left(\overline{\alpha_{4}}, \alpha_{3}, \alpha_{s}\right)}{M\left(\overline{\alpha_{4}}, \alpha_{t}, \alpha_{1}\right) M\left(\alpha_{4}, \alpha_{3}, \alpha_{s}\right)} F_{\alpha_{s} \alpha_{t}}^{\mathrm{PT}}\left[\begin{array}{c}
\alpha_{3} \alpha_{2} \\
\overline{\alpha_{4}} \alpha_{1}
\end{array}\right] .
$$


Then

$$
\begin{aligned}
F_{\alpha_{s} \alpha_{t}}^{\mathrm{L}}\left[\begin{array}{c}
\bar{\alpha} \alpha \\
\alpha \bar{\alpha}
\end{array}\right]= & \frac{\Gamma_{b}\left(2 \alpha_{s}\right)}{\Gamma_{b}\left(\alpha_{s}\right)} \frac{\Gamma_{b}\left(\alpha_{t}\right)}{\Gamma_{b}\left(2 \alpha_{t}\right)} \frac{\Gamma_{b}\left(2 Q-2 \alpha_{s}\right) \Gamma_{b}\left(Q-\alpha_{t}\right)^{3}}{\Gamma_{b}\left(2 \alpha-\alpha_{s}\right) \Gamma_{b}\left(Q-\alpha_{s}\right)^{3}} \frac{\Gamma_{b}\left(2 \alpha-\alpha_{t}\right) \Gamma_{b}\left(-2 \alpha+Q+\alpha_{t}\right) \Gamma_{b}\left(2 \alpha-Q+\alpha_{t}\right)^{2}}{\Gamma_{b}\left(-2 \alpha+Q+\alpha_{s}\right) \Gamma_{b}\left(2 \alpha-Q+\alpha_{s}\right)^{2} \Gamma_{b}\left(2 Q-2 \alpha_{t}\right)} \\
& \times \frac{\Gamma_{b}\left(\alpha_{t}\right)}{\Gamma_{b}\left(\alpha_{s}\right)} \frac{\Gamma_{b}\left(Q-\alpha_{s}\right) \Gamma_{b}\left(2 \alpha-Q+\alpha_{s}\right) \Gamma_{b}\left(-2 \alpha+2 Q-\alpha_{t}\right)}{\Gamma_{b}\left(-2 \alpha+2 Q-\alpha_{s}\right) \Gamma_{b}\left(Q-\alpha_{t}\right) \Gamma_{b}\left(2 \alpha-Q+\alpha_{t}\right)} \frac{\left.\Gamma_{b}\right) \Gamma_{b}\left(-2 \alpha+Q+\alpha_{s}\right) \Gamma_{b}\left(2 \alpha-Q+\alpha_{s}\right)}{\Gamma_{b}\left(2 \alpha-\alpha_{s}\right) \Gamma_{b}\left(Q-\alpha_{s}\right) \Gamma_{b}\left(-2 \alpha+2 Q-\alpha_{s}\right)} \\
& \times\left|S_{b}\left(2 \alpha_{t}\right)\right|^{2} .
\end{aligned}
$$

The factor in the second line of (A78) comes from the four normalization factors $N\left(\alpha_{3}, \alpha_{2}, \alpha_{1}\right)$ in Eq. (A70), the factor in the third line of (A78) is from the four factors associated with $M\left(\alpha_{3}, \alpha_{2}, \alpha_{1}\right)$ in Eq. (A77), and the factor in the last line of (A78) is mainly from $F_{\alpha_{s} \alpha_{t}}^{\mathrm{PT}}\left[\begin{array}{c}\alpha_{3} \alpha_{2} \\ \alpha_{4} \alpha_{1}\end{array}\right]$ in Eq. (A72). We have already made use of Eq. (A73) to do the $u$ integration to obtain the simple expression (A78). From (A78) and the meromorphic property of $\Gamma_{b}(\alpha)$ shown in the Appendix, one can see that there is no pole structure in $F_{\alpha_{s} \alpha_{t}}^{\mathrm{L}}\left[\begin{array}{c}\bar{\alpha} \alpha \\ \alpha \bar{\alpha}\end{array}\right]$ for $\alpha_{s} \rightarrow 0$. We then obtain the following expansion near $\alpha_{s} \rightarrow 0$,

$F_{0 \alpha_{t}}^{\mathrm{L}}\left[\begin{array}{l}\alpha_{2} \alpha_{1} \\ \alpha_{2} \alpha_{1}\end{array}\right]=\frac{1}{2}\left|S_{b}\left(2 \alpha_{t}\right)\right|^{2} \frac{\Gamma_{b}\left(\alpha_{t}\right)^{2}}{\Gamma_{b}\left(2 \alpha_{t}\right)} \frac{\Gamma_{b}(2 Q) \Gamma_{b}\left(Q-\alpha_{t}\right)^{2} \Gamma_{b}\left(-2 \alpha+2 Q-\alpha_{t}\right)}{\Gamma_{b}(Q)^{3} \Gamma_{b}(2 Q-2 \alpha)^{2}} \frac{\Gamma_{b}\left(2 \alpha-\alpha_{t}\right) \Gamma_{b}\left(-2 \alpha+Q+\alpha_{t}\right) \Gamma_{b}\left(2 \alpha-Q+\alpha_{t}\right)}{\Gamma_{b}(2 \alpha)^{2} \Gamma_{b}\left(2 Q-2 \alpha_{t}\right)}$.

The factor $\left|S_{b}\left(2 \alpha_{t}\right)\right|^{2}$ can be taken care of using

$$
\left|S_{b}(\alpha)\right|^{2}=-4 \sin \pi b(2 \alpha-Q) \sin \pi b^{-1}(2 \alpha-Q),
$$

but we will temporarily keep it. The only divergence is from the simple pole of $\Gamma_{b}\left(\alpha_{t}\right)$ in Eq. (A34),

$$
\Gamma_{b}(x) \sim \frac{\Gamma_{b}(Q)}{2 \pi x},
$$

and the residue is given by the simple pole of $F_{0 \alpha_{t} \rightarrow 0}^{L}\left[\begin{array}{c}\bar{\alpha} \alpha \\ \alpha \bar{\alpha}\end{array}\right]$,

$$
F_{0 \alpha_{t} \rightarrow 0}^{L}\left[\begin{array}{l}
\bar{\alpha} \alpha \\
\alpha \bar{\alpha}
\end{array}\right]=\frac{1}{2 \pi}\left|S_{b}(\alpha)\right|^{2} \cdot \frac{\Gamma_{b}(Q-2 \alpha) \Gamma_{b}(2 \alpha-Q)}{\Gamma_{b}(2 \alpha) \Gamma_{b}(2 Q-2 \alpha)}=\frac{1}{2 \pi}\left|S_{b}(\alpha)\right|^{2} \cdot \frac{S_{b}(2 \alpha-Q)}{S_{b}(2 \alpha)} .
$$

With the help of the following identity from Eq. (A37),

$$
S_{b}\left(x+b^{ \pm 1}\right)=2 \sin \left(\pi b^{ \pm 1} x\right) S_{b}(x),
$$

we can express the residue as [where we also use $S_{b}(x)=S_{b^{-1}}(x)$ ]

$F_{0 \alpha_{t} \rightarrow 0}\left[\begin{array}{c}\bar{\alpha} \alpha \\ \alpha \bar{\alpha}\end{array}\right]=\frac{1}{2 \pi \alpha_{t}} \frac{\left|S_{b}\left(2 \alpha_{t}\right)\right|^{2} S_{b}(2 \alpha-Q)}{4 \sin \left[\pi b\left(2 \alpha-Q+\frac{1}{b}\right)\right] \sin \left[\pi b^{-1}(2 \alpha-Q)\right] S_{b}(2 \alpha-Q)}=\frac{1}{2 \pi \alpha_{t}} \frac{\sin \pi b Q \sin \pi b^{-1} Q}{\sin [\pi b(2 \alpha-Q)] \sin \left[\pi b^{-1}(2 \alpha-Q)\right]}$.

By comparing the definition of the quantum dimension [40] in LFT, we will show that the $F_{0,0}^{L}\left[\begin{array}{c}\bar{\alpha} \alpha \\ \alpha \bar{\alpha}\end{array}\right]$ will be the quantum dimension.

\section{The Fusion Matrix in Super-Liouville Field Theory}

Similar to Appendix A 7, we would like to comment on the quantum dimension and fusion matrix presented in the second REE in SLFT. Generically, the fusion matrices take the form $i, j=1,2$, which correspond to the parity of $e$ and $o$, respectively [48]: 


$$
\begin{aligned}
F_{\alpha_{s}, \alpha_{t}}\left[\begin{array}{cc}
\alpha_{3} & \alpha_{2} \\
\alpha_{4} & \alpha_{1}
\end{array}\right]_{j}^{i}= & \frac{\Gamma_{i}\left(2 Q-\alpha_{t}-\alpha_{2}-\alpha_{3}\right) \Gamma_{i}\left(Q-\alpha_{t}+\alpha_{3}-\alpha_{2}\right) \Gamma_{i}\left(Q+\alpha_{t}-\alpha_{2}-\alpha_{3}\right) \Gamma_{i}\left(\alpha_{3}+\alpha_{t}-\alpha_{2}\right)}{\Gamma_{j}\left(2 Q-\alpha_{1}-\alpha_{s}-\alpha_{2}\right) \Gamma_{j}\left(Q-\alpha_{s}-\alpha_{2}+\alpha_{1}\right) \Gamma_{j}\left(Q-\alpha_{1}-\alpha_{2}+\alpha_{s}\right) \Gamma_{j}\left(\alpha_{s}+\alpha_{1}-\alpha_{2}\right)} \\
& \times \frac{\Gamma_{i}\left(Q-\alpha_{t}-\alpha_{1}+\alpha_{4}\right) \Gamma_{i}\left(\alpha_{1}+\alpha_{4}-\alpha_{t}\right) \Gamma_{i}\left(\alpha_{t}+\alpha_{4}-\alpha_{1}\right) \Gamma_{i}\left(\alpha_{t}+\alpha_{1}+\alpha_{4}-Q\right)}{\Gamma_{j}\left(Q-\alpha_{s}-\alpha_{3}+\alpha_{4}\right) \Gamma_{j}\left(\alpha_{3}+\alpha_{4}-\alpha_{s}\right) \Gamma_{j}\left(\alpha_{s}+\alpha_{4}-\alpha_{3}\right) \Gamma_{j}\left(\alpha_{s}+\alpha_{3}+\alpha_{4}-Q\right)} \\
& \times \frac{\Gamma_{\mathrm{NS}}\left(2 Q-2 \alpha_{s}\right) \Gamma_{\mathrm{NS}}\left(2 \alpha_{s}\right)}{\Gamma_{\mathrm{NS}}\left(Q-2 \alpha_{t}\right) \Gamma_{\mathrm{NS}}\left(2 \alpha_{t}-Q\right)} \frac{1}{i} \int_{-i \infty}^{i \infty} d \tau J_{\alpha_{s}, \alpha_{t}}\left[\begin{array}{cc}
\alpha_{3} & \alpha_{2} \\
\alpha_{4} & \alpha_{1}
\end{array}\right]_{j}^{i} .
\end{aligned}
$$

We will consider $i=j=1$, which gives the fusion matrix for the NS sector. In this case, we have

$$
\begin{aligned}
J_{\alpha_{s}, \alpha_{t}}\left[\begin{array}{cc}
\alpha_{3} & \alpha_{2} \\
\alpha_{4} & \alpha_{1}
\end{array}\right]_{1}^{1}= & \frac{S_{\mathrm{NS}}\left(Q+\tau-\alpha_{1}\right) S_{\mathrm{NS}}\left(\tau+\alpha_{4}+\alpha_{2}-\alpha_{3}\right) S_{\mathrm{NS}}\left(\tau+\alpha_{1}\right) S_{\mathrm{NS}}\left(\tau+\alpha_{4}+\alpha_{2}+\alpha_{3}-Q\right)}{S_{\mathrm{NS}}\left(Q+\tau+\alpha_{4}-\alpha_{t}\right) S_{\mathrm{NS}}\left(\tau+\alpha_{4}+\alpha_{t}\right) S_{\mathrm{NS}}\left(Q+\tau+\alpha_{2}-\alpha_{s}\right) S_{\mathrm{NS}}\left(\tau+\alpha_{2}+\alpha_{s}\right)} \\
& +\frac{S_{R}\left(Q+\tau-\alpha_{1}\right) S_{R}\left(\tau+\alpha_{4}+\alpha_{2}-\alpha_{3}\right) S_{R}\left(\tau+\alpha_{1}\right) S_{R}\left(\tau+\alpha_{4}+\alpha_{2}+\alpha_{3}-Q\right)}{S_{R}\left(Q+\tau+\alpha_{4}-\alpha_{t}\right) S_{R}\left(\tau+\alpha_{4}+\alpha_{t}\right) S_{R}\left(Q+\tau+\alpha_{2}-\alpha_{s}\right) S_{R}\left(\tau+\alpha_{2}+\alpha_{s}\right)}
\end{aligned}
$$

where $S_{\mathrm{NS}, R}$ are defined by Eq. (A41). In terms of the explicit form of

$$
F_{\alpha_{s}, \alpha_{t}}\left[\begin{array}{cc}
\alpha_{3} & \alpha_{2} \\
\alpha_{4} & \alpha_{1}
\end{array}\right]
$$

in Eq. (A84), we can show that

$$
F_{\alpha_{s}, \alpha_{t}}\left[\begin{array}{cc}
\bar{\alpha} & \alpha \\
\alpha & \bar{\alpha}
\end{array}\right]_{1}^{1}=F_{\alpha_{s}, \alpha_{t}}\left[\begin{array}{cc}
\alpha & \alpha \\
\alpha & \alpha
\end{array}\right]_{1}^{1}
$$

Alternatively, it can be shown that for $\alpha_{s}=0$, the fusion matrix becomes

$$
\begin{aligned}
F_{0, \alpha_{t}}\left[\begin{array}{ll}
\alpha_{3} & \alpha_{1} \\
\alpha_{3} & \alpha_{1}
\end{array}\right]_{1}^{1}= & C_{\mathrm{NS}}\left(\alpha_{t}, \alpha_{1}, \alpha_{3}\right) \\
& \times \frac{W_{\mathrm{NS}}(Q) W_{\mathrm{NS}}\left(\alpha_{t}\right)}{\pi W_{\mathrm{NS}}\left(Q-\alpha_{1}\right) W_{\mathrm{NS}}\left(Q-\alpha_{3}\right)},
\end{aligned}
$$

where $W_{\mathrm{NS}}$ is defined in (A45) and (A46). It is not difficult to see that near $\alpha_{t} \sim 0$ (and $\alpha_{1}=\alpha_{3}=\alpha$ ), the DOZZ function has a single pole,

$$
\begin{aligned}
C_{\mathrm{NS}}\left(\alpha_{t}, \alpha, \alpha\right) & \sim \lambda^{(Q-2 \alpha) / b} \frac{\Upsilon_{\mathrm{NS}}^{\prime}(0) \Upsilon_{\mathrm{NS}}\left(2 \alpha_{t}\right) \Upsilon_{\mathrm{NS}}(2 \alpha)}{\Upsilon_{\mathrm{NS}}(2 \alpha-Q) \Upsilon_{\mathrm{NS}}\left(\alpha_{t}\right)^{2}}, \\
& \sim \frac{\Upsilon_{\mathrm{NS}}^{\prime}(0) \Upsilon_{\mathrm{NS}}\left(2 \alpha_{t}\right) W_{\mathrm{NS}}(Q-\alpha)}{W_{\mathrm{NS}}(\alpha) \Upsilon_{\mathrm{NS}}^{2}\left(\alpha_{t}\right)} \\
& \sim \frac{2 W_{\mathrm{NS}}(Q-\alpha)}{W_{\mathrm{NS}}(\alpha) \pi \alpha_{t}},
\end{aligned}
$$

where in the second line we use

$$
\Gamma_{\mathrm{NS}}(x) \sim \frac{\Gamma_{\mathrm{NS}}(Q)}{\pi x} \Rightarrow \Upsilon_{\mathrm{NS}}(x) \sim \frac{\pi x}{\Gamma_{\mathrm{NS}}^{2}(Q)} .
$$

In the second line of (A88) we use

$$
\frac{\Upsilon_{\mathrm{NS}}(2 x)}{\Upsilon_{\mathrm{NS}}(2 x-Q)}=\mathcal{G}_{\mathrm{NS}}(x) \lambda^{-\frac{Q-2 x}{b}}=\frac{W_{\mathrm{NS}}(Q-x)}{W_{\mathrm{NS}}(x)} \lambda^{-\frac{Q-2 x}{b}}
$$

We also need the values of the derivative $\Upsilon_{\mathrm{NS}}^{\prime}(0)$ :

$$
\Upsilon_{\mathrm{NS}}^{\prime}(0)=\frac{\pi}{\Gamma_{\mathrm{NS}}^{2}(Q)}
$$

We have made use of the definition of $\Gamma_{N S}, \Gamma_{R}$ given in Eq. (A41). Substituting (A88) back into (A87) and with the help of the identity

$$
W_{\mathrm{NS}}(x) W_{\mathrm{NS}}(Q-x)=-4 \sin \pi b(x-Q / 2) \sin \pi \frac{1}{b}(x-Q / 2),
$$

we obtain the pole structure of the fusion matrix:

$F_{0, \alpha_{t}}\left[\begin{array}{ll}\alpha & \alpha \\ \alpha & \alpha\end{array}\right]_{1}^{1}=\frac{2}{\pi^{2} \alpha_{t}} \frac{\sin \frac{\pi}{2} b Q \sin \frac{\pi}{2} b^{-1} Q b(\alpha-Q / 2) \sin \pi b^{-1}(\alpha-Q / 2)}{\sin }$.

So we can again relate the entanglement entropy due to the local operator to its quantum dimension

$\operatorname{Res}_{\alpha_{t}=0} F_{00} \sim \sin \pi b(\alpha-Q / 2) \sin \pi b^{-1}(\alpha-Q / 2)$.

Similar to the situation in LFT, the fusion matrix element for $F_{Q / 2, Q / 2}^{S L}\left[\begin{array}{c}\alpha \alpha \\ \alpha \alpha\end{array}\right]_{e}$ p presented in the second REE (56) in SLFT cannot be identified as a quantum dimension. 
[1] A. Kitaev and J. Preskill, Topological Entanglement Entropy, Phys. Rev. Lett. 96, 110404 (2006); M. Levin and X.G. Wen, Detecting Topological Order in a Ground State Wave Function, Phys. Rev. Lett. 96, 110405 (2006).

[2] P. Fendley, M. P. A. Fisher, and C. Nayak, Topological entanglement entropy from the holographic partition function, J. Stat. Phys. 126, 1111 (2007).

[3] P. Calabrese and J. L. Cardy, Entanglement entropy and quantum field theory, J. Stat. Mech. (2004) P06002.

[4] S. He, T. Numasawa, T. Takayanagi, and K. Watanabe, Quantum dimension as entanglement entropy in 2D CFTs, Phys. Rev. D 90, 041701 (2014).

[5] F. C. Alcaraz, M. I. Berganza, and G. Sierra, Entanglement of Low-Energy Excitations in Conformal Field Theory, Phys. Rev. Lett. 106, 201601 (2011).

[6] T. Pálmai, Excited state entanglement in one dimensional quantum critical systems: Extensivity and the role of microscopic details, Phys. Rev. B 90, 161404 (2014).

[7] M. Nozaki, T. Numasawa, and T. Takayanagi, Quantum Entanglement of Local Operators in Conformal Field Theories, Phys. Rev. Lett. 112, 111602 (2014).

[8] M. Nozaki, Notes on quantum entanglement of local operators, J. High Energy Phys. 10 (2014) 147.

[9] N. Shiba, Entanglement entropy of disjoint regions in excited states: An operator method, J. High Energy Phys. 12 (2014) 152.

[10] P. Caputa, M. Nozaki, and T. Takayanagi, Entanglement of local operators in large-N conformal field theories, Prog. Theor. Exp. Phys. 2014, 093 B06 (2014).

[11] C. T. Asplund, A. Bernamonti, F. Galli, and T. Hartman, Holographic entanglement entropy from 2D CFT: Heavy states and local quenches, J. High Energy Phys. 02 (2015) 171.

[12] G. W. Moore and N. Seiberg, Polynomial equations for rational conformal field theories, Phys. Lett. B 212, 451 (1988); Classical and quantum conformal field theory, Commun. Math. Phys. 123, 177 (1989).

[13] E. P. Verlinde, Fusion rules and modular transformations in 2D conformal field theory, Nucl. Phys. B300, 360 (1988); J. L. Cardy, Boundary conditions, fusion rules and the Verlinde formula, Nucl. Phys. B324, 581 (1989); R. Dijkgraaf and E. P. Verlinde, Modular invariance and the fusion algebra, Nucl. Phys. B, Proc. Suppl. 5, 87 (1988).

[14] S. He, T. Numasawa, T. Takayanagi, and K. Watanabe, Notes on entanglement entropy in string theory, J. High Energy Phys. 05 (2015) 106.

[15] W. Z. Guo and S. He, Rényi entropy of locally excited states with thermal and boundary effect in 2D CFTs, J. High Energy Phys. 04 (2015) 099.

[16] M. Nozaki, T. Numasawa, and S. Matsuura, Quantum entanglement of fermionic local operators, J. High Energy Phys. 02 (2016) 150.

[17] P. Caputa, T. Numasawa, and A. Veliz-Osorio, Scrambling without chaos in rational conformal field theories, Prog. Theor. Exp. Phys. 2016, 113 B06 (2016).

[18] B. Chen and J. q. Wu, Holographic entanglement entropy for a general state in 2D CFT, J. High Energy Phys. 09 (2016) 015.

[19] M. Nozaki and N. Watamura, Quantum entanglement of locally excited states in Maxwell theory, J. High Energy Phys. 12 (2016) 069.
[20] T. G. Mertens, H. Verschelde, and V. I. Zakharov, String theory in polar coordinates and the vanishing of the one-loop Rindler entropy, J. High Energy Phys. 08 (2016) 113.

[21] T. Zhou, X. Chen, T. Faulkner, and E. Fradkin, Entanglement entropy and mutual information of circular entangling surfaces in 2+1-dimensional quantum Lifshitz model, J. Stat. Mech. (2016) 093101.

[22] T. Zhou, Entanglement entropy of local operators in quantum Lifshitz theory, J. Stat. Mech. (2016) 093106.

[23] P. Caputa and M. M. Rams, Quantum dimensions from local operator excitations in the Ising model, J. Phys. A 50, 055002 (2017).

[24] F. L. Lin, H. Wang, and J. j. Zhang, Thermality and excited state Rényi entropy in two-dimensional CFT, J. High Energy Phys. 11 (2016) 116.

[25] N. Shiba, Aharonov-Bohm effect on entanglement entropy in conformal field theory, Phys. Rev. D 96, 065016 (2017).

[26] P. Caputa, Y. Kusuki, T. Takayanagi, and K. Watanabe, Evolution of entanglement entropy in Orbifold CFTs, J. Phys. A 50, 244001 (2017).

[27] S. He, F. L. Lin, and J. j. Zhang, Subsystem eigenstate thermalization hypothesis for entanglement entropy in CFT, J. High Energy Phys. 08 (2017) 126.

[28] A. Jahn and T. Takayanagi, Holographic entanglement entropy of local quenches in $\mathrm{AdS}_{4} / \mathrm{CFT}_{3}$ : A finite-element approach, J. Phys. A 51, 015401 (2018).

[29] S. He, F. L. Lin, and J. j. Zhang, Dissimilarities of reduced density matrices and eigenstate thermalization hypothesis, J. High Energy Phys. 12 (2017) 073.

[30] V. Balasubramanian, A. Bernamonti, B. Craps, T. De Jonckheere, and F. Galli, Heavy-heavy-light-light correlators in Liouville theory, J. High Energy Phys. 08 (2017) 045.

[31] Z. X. Luo and H. Y. Sun, Topological entanglement entropy in Euclidean $\mathrm{AdS}_{3}$ via surgery, J. High Energy Phys. 12 (2017) 116.

[32] X. Wen, Y. Wang, and S. Ryu, Entanglement evolution across a conformal interface, J. Phys. A 51, 195004 (2018).

[33] Y. Kusuki and T. Takayanagi, Renyi entropy for local quenches in 2D CFT from numerical conformal blocks, J. High Energy Phys. 01 (2018) 115.

[34] W. Z. Guo, S. He, and Z. X. Luo, Entanglement entropy in $(1+1)$ D CFTs with multiple local excitations, J. High Energy Phys. 05 (2018) 154.

[35] T. Numasawa, Scattering effect on entanglement propagation in RCFTs, J. High Energy Phys. 12 (2016) 061.

[36] B. Chen, W.Z. Guo, S. He, and J.q. Wu, Entanglement entropy for descendent local operators in 2D CFTs, J. High Energy Phys. 10 (2015) 173.

[37] C. T. Asplund, A. Bernamonti, F. Galli, and T. Hartman, Entanglement scrambling in $2 \mathrm{~d}$ conformal field theory, J. High Energy Phys. 09 (2015) 110.

[38] D. Harlow, J. Maltz, and E. Witten, Analytic continuation of Liouville theory, J. High Energy Phys. 12 (2011) 071.

[39] H. L. Verlinde, Conformal field theory, 2-D quantum gravity and quantization of Teichmuller space, Nucl. Phys. B337, 652 (1990).

[40] L. McGough and H. Verlinde, Bekenstein-Hawking entropy as topological entanglement entropy, J. High Energy Phys. 11 (2013) 208. 
[41] S. Jackson, L. McGough, and H. Verlinde, Conformal bootstrap, universality and gravitational scattering, Nucl. Phys. B901, 382 (2015).

[42] D. Gaiotto and E. Witten, Knot invariants from fourdimensional gauge theory, Adv. Theor. Math. Phys. 16, 935 (2012).

[43] N. Seiberg, Notes on quantum Liouville theory and quantum gravity, Prog. Theor. Phys. Suppl. 102, 319 (1990).

[44] E. D'Hoker and R. Jackiw, Classical and quantal Liouville field theory, Phys. Rev. D 26, 3517 (1982).

[45] W. McElgin, Notes on Liouville theory at $c<=1$, Phys. Rev. D 77, 066009 (2008).

[46] Y. Nakayama, Liouville field theory: A decade after the revolution, Int. J. Mod. Phys. A 19, 2771 (2004).

[47] L. Hadasz, Z. Jaskolski, and P. Suchanek, Elliptic recurrence representation of the $N=1$ superconformal blocks in the Ramond sector, J. High Energy Phys. 11 (2008) 060.

[48] H. Poghosyan and G. Sarkissian, Comments on fusion matrix in $N=1$ super Liouville field theory, Nucl. Phys. B909, 458 (2016).

[49] G. W. Moore and N. Seiberg, Naturality in conformal field theory, Nucl. Phys. B313, 16 (1989).

[50] M. R. Gaberdiel and R. Gopakumar, Minimal model holography, J. Phys. A 46, 214002 (2013).

[51] D. A. Roberts and D. Stanford, Two-Dimensional Conformal Field Theory and the Butterfly Effect, Phys. Rev. Lett. 115, 131603 (2015).

[52] T. G. Mertens, G. J. Turiaci, and H. L. Verlinde, Solving the Schwarzian via the conformal bootstrap, J. High Energy Phys. 08 (2017) 136.

[53] P. Caputa, N. Kundu, M. Miyaji, T. Takayanagi, and K. Watanabe, Anti-de Sitter Space from Optimization of Path Integrals in Conformal Field Theories, Phys. Rev. Lett. 119, 071602 (2017).

[54] B. Czech, Einstein's Equations from Varying Complexity, Phys. Rev. Lett. 120, 031601 (2018).

[55] P. Caputa, N. Kundu, M. Miyaji, T. Takayanagi, and K. Watanabe, Liouville action as path-integral complexity: From continuous tensor networks to AdS/CFT, J. High Energy Phys. 11 (2017) 097.

[56] R. A. Jefferson and R. C. Myers, Circuit complexity in quantum field theory, J. High Energy Phys. 10 (2017) 107.

[57] S. Chapman, M. P. Heller, H. Marrochio, and F. Pastawski, Towards Complexity for Quantum Field Theory States, Phys. Rev. Lett. 120, 121602 (2018).

[58] L. Susskind, Computational complexity and black hole horizons, Fortschr. Phys. 64, 24 (2016); 64, 44(A) (2016).

[59] M. Alishahiha, Holographic complexity, Phys. Rev. D 92, 126009 (2015).

[60] A. R. Brown, D. A. Roberts, L. Susskind, B. Swingle, and Y. Zhao, Complexity, action, and black holes, Phys. Rev. D 93, 086006 (2016).
[61] A. R. Brown, D. A. Roberts, L. Susskind, B. Swingle, and Y. Zhao, Holographic Complexity Equals Bulk Action?, Phys. Rev. Lett. 116, 191301 (2016).

[62] R. Abt, J. Erdmenger, H. Hinrichsen, C. M. MelbyThompson, R. Meyer, C. Northe, and I. A. Reyes, Topological complexity in $\mathrm{AdS}_{3} / \mathrm{CFT}_{2}$, Fortschr. Phys. 66, 1800034 (2018).

[63] H. Dorn and H. J. Otto, Two and three point functions in Liouville theory, Nucl. Phys. B429, 375 (1994).

[64] A. B. Zamolodchikov and A. B. Zamolodchikov, Structure constants and conformal bootstrap in Liouville field theory, Nucl. Phys. B477, 577 (1996).

[65] L. Hadasz, Z. Jaskolski, and P. Suchanek, Recursion representation of the Neveu-Schwarz superconformal block, J. High Energy Phys. 03 (2007) 032.

[66] A. Belavin, V. Belavin, A. Neveu, and A. Zamolodchikov, Bootstrap in supersymmetric Liouville field theory. I. NS sector, Nucl. Phys. B784, 202 (2007).

[67] R. Poghossian, Structure constants in the $N=1$ superLiouville field theory. Nucl. Phys. B496, 451 (1997).

[68] R. Rashkov and M. Stanishkov, Three point correlation functions in $N=1$ super Liouville theory, Phys. Lett. B 380, 49 (1996).

[69] E. W. Barnes, Theory of the double gamma function, Phil. Trans. R. Soc. A 196, 265 (1901).

[70] M. Spreafico, On the Barnes double zeta and gamma functions, J. Number Theory 129, 2035 (2009).

[71] T. Fukuda and K. Hosomichi, Super Liouville theory with boundary, Nucl. Phys. B635, 215 (2002).

[72] X. Cao, P. Le Doussal, A. Rosso, and R. Santachiara, Liouville Field Theory and Log-Correlated Random Energy Models, Phys. Rev. Lett. 118, 090601 (2017).

[73] A. Zamolodchikov, Gravitational Yang-Lee model: Four point function, Theor. Math. Phys. 151, 439 (2007).

[74] A. A. Belavin and A. B. Zamolodchikov, Integrals over moduli spaces, ground ring, and four-point function in minimal Liouville gravity, Theor. Math. Phys. 147, 729 (2006).

[75] K. Aleshkin and V. Belavin, On the construction of the correlation numbers in minimal Liouville gravity, J. High Energy Phys. 11 (2016) 142.

[76] S. Ribault, Conformal field theory on the plane, arXiv: 1406.4290.

[77] J. Teschner and G.S. Vartanov, Supersymmetric gauge theories, quantization of $\mathcal{M}_{\mathrm{flat}}$, and conformal field theory, Adv. Theor. Math. Phys. 19, 1 (2015).

[78] J. Teschner, Liouville theory revisited, Classical Quantum Gravity 18, R153 (2001).

[79] B. Ponsot and J. Teschner, Liouville bootstrap via harmonic analysis on a noncompact quantum group, arXiv:hep-th/ 9911110.

[80] J. Teschner and G. Vartanov, 6j symbols for the modular double, quantum hyperbolic geometry, and supersymmetric gauge theories, Lett. Math. Phys. 104, 527 (2014). 